\title{
THE WAVE EQUATION WITH OSCILLATING DENSITY: OBSERVABILITY AT LOW FREQUENCY
}

\author{
Gilles LeBeAU ${ }^{1}$
}

\begin{abstract}
We prove an observability estimate for a wave equation with rapidly oscillating density, in a bounded domain with Dirichlet boundary condition.
\end{abstract}

AMS Subject Classification. 35L05, 35L20, 35S15, 93B07.

Received July 8, 1999. Revised March 6, 2000.

\section{INTRODUCTION AND RESULTS}

Let $\Omega$ be a smooth bounded domain in $\mathbb{R}^{d}$, and $\rho(x, y)$ a smooth function on $\mathbb{R}^{d} \times \mathbb{R}^{d}$, such that

$$
0<\rho_{\min } \leq \rho(x, y) \leq \rho_{\max } \quad \forall(x, y)
$$

$\rho$ is $2 \pi$-periodic with respect to the second variable, i.e.

$$
\rho(x, y)=\rho(x, y+2 \pi \ell) \quad \forall \ell \in \mathbb{Z}^{d} .
$$

For $\varepsilon>0$, let $\left(\omega_{n}^{\varepsilon}, e_{n}^{\varepsilon}(x)\right)$ be the spectrum of the Dirichlet problem for the operator $-\rho^{-1}(x, x / \varepsilon) \Delta_{g}$ on $L^{2}\left(\Omega ; \rho(x, x / \varepsilon) d_{g} x\right)$ normalized in the form

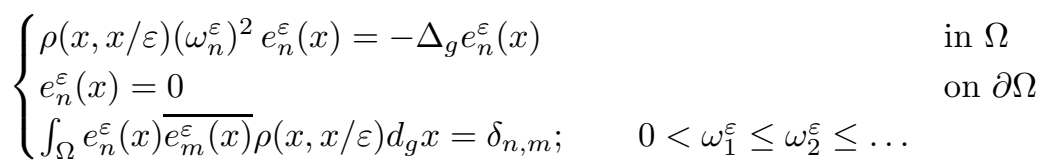

Here, $\Delta_{g}$ denotes the Laplace operator for some fixed smooth metric $g$ on $\bar{\Omega}$, and $d_{g} x$ is the volume form associated to $g$.

For any given $\gamma_{0}>0$, we shall denote by $J_{\gamma_{0}}^{\varepsilon}$ the space of solutions $u^{\varepsilon}(t, x)$ of the wave equation with oscillating density $\rho$

$$
\left\{\begin{array}{l}
\left(\rho(x, x / \varepsilon) \partial_{t}^{2}-\Delta_{g}\right) u^{\varepsilon}(t, x)=0 \quad \text { in } \mathbb{R}_{+} \times \Omega \\
u^{\varepsilon}(t, x)_{\mid x \in \partial \Omega}=0
\end{array}\right.
$$

Keywords and phrases: Bloch wave, observability, microlocal defect measures.

1 Centre de Mathématiques, École Polytechnique, UMR 7640 du CNRS, 91128 Palaiseau Cedex, France;

e-mail: lebeau@math.polytechnique.fr

(C) EDP Sciences, SMAI 2000 
with maximum frequency less than $\gamma_{0} / \varepsilon$.

In other words, $J_{\gamma_{0}}^{\varepsilon}$ is the set

$$
J_{\gamma_{0}}^{\varepsilon}=\left\{u^{\varepsilon}(t, x)=\sum_{\varepsilon \omega_{n}^{\varepsilon} \leq \gamma_{0}}\left(u_{+, n} e^{i t \omega_{n}^{\varepsilon}}+u_{-, n} e^{-i t \omega_{n}^{\varepsilon}}\right) e_{n}^{\varepsilon}(x)\right\} .
$$

Let $\left\{u_{k}^{\varepsilon_{k}}\right\}$ be a bounded sequence (in $L_{\text {loc }}^{2}\left(\mathbb{R}_{t}, L^{2}(\Omega)\right.$ ), of solutions of (0.4), with lim $\varepsilon_{k}=0$. It is well known that any weak limit of this sequence will satisfy the homogenized wave equation in $\Omega$

$$
\left\{\begin{array}{l}
\left(\underline{\rho}(x) \partial_{t}^{2}-\Delta_{g}\right) u(t, x)=0 \quad \text { in } \mathbb{R} \times \Omega \\
u(t, x)_{\mid x \in \partial \Omega}=0
\end{array}\right.
$$

where $\rho(x)=\oint \rho(x, y) d y$ is the mean value of $\rho$.

Let $\bar{V}$ be an open subset of $\Omega$, and $T_{0}>0$.

One says that waves solution of (0.6) are observable from $V$ in time $T_{0}$ if there exists a constant $C_{0}$ s.t for any $L^{2}$-solution of $(0.6)$ one has

$$
\int_{0}^{T_{0}} \int_{\Omega}|u(t, x)|^{2} \underline{\rho}(x) d t d_{g} x \leq C_{0} \int_{0}^{T_{0}} \int_{V}|u(t, x)|^{2} \underline{\rho}(x) d t d_{g} x .
$$

If $u=\sum_{ \pm, n} u_{ \pm, n} e^{ \pm i t \omega_{n}} e_{n}(x)$ is the Fourier series of $u$ in the spectral decomposition of $(-\underline{\rho})^{-1}(x) \Delta_{g}$, we deduce from the elementary fact

$$
\forall T>0, \forall \omega_{0}>0, \exists C>0 \text { such that } \forall \omega \geq \omega_{0},\left|c_{+}\right|^{2}+\left|c_{-}\right|^{2} \leq C \int_{0}^{T}\left|c_{+} e^{i t \omega}+c_{-} e^{-i t \omega}\right|^{2} d t
$$

that the condition (0.7) is equivalent to the following

$$
\left\{\begin{array}{l}
\exists C_{0} \text { s.t. } \forall\left(u_{+, n}, u_{-, n}\right)_{n} \in \ell^{2} \times \ell^{2} \\
\sum_{n}\left|u_{+, n}\right|^{2}+\left|u_{-, n}\right|^{2} \leq C_{0} \int_{0}^{T_{0}} \int_{V}|u(t, x)|^{2} \underline{\rho}(x) d t d_{g} x .
\end{array}\right.
$$

It is proved in [4] that (0.7) holds true under the geometric-control hypothesis

$$
\left\{\begin{array}{l}
\text { 1) there is no infinite order of contact between the boundary } \\
\partial \Omega \text { and the bicharacteristics of } \rho(x) \partial_{t}^{2}-\Delta_{g} \\
\text { 2) any generalized bicharacteristic of } \rho(x) \partial_{t}^{2}-\Delta_{g} \\
\text { parameterized by } t \in] 0, T_{0}[\text { meets } \bar{V} \text {. }
\end{array}\right.
$$

Here the generalized bicharacteristic flow is the one defined by Melrose and Sjöstrand in [11].

The main result of this paper is the following theorem, which asserts that the estimate (0.7) remains true under the hypothesis (0.9) for $\underline{\rho}(x)$, for solutions of (0.4) in $J_{\gamma_{0}}^{\varepsilon}$, if $\gamma_{0}$ is small enough.

Theorem 0.1. Let the hypothesis (0.9) be satisfied. There exist small positive constants $\gamma_{0}, \varepsilon_{0}$ and a constant $C_{0}$, such that for any $\left.\varepsilon \in\right] 0, \varepsilon_{0}\left[\right.$ and any $u^{\varepsilon} \in J_{\gamma_{0}}^{\varepsilon}$

$$
\int_{0}^{T_{0}} \int_{\Omega}\left|u^{\varepsilon}(t, x)\right|^{2} \rho(x, x / \varepsilon) d t d_{g} x \leq C_{0} \int_{0}^{T_{0}} \int_{V}\left|u^{\varepsilon}(t, x)\right|^{2} \rho(x, x / \varepsilon) d t d_{g} x
$$


This is clearly a stability result of the observability estimate (0.7) under the singular perturbation $\rho(x) \rightarrow \rho(x, x / \varepsilon)$. Let us recall that Theorem 0.1 has been proved in the 1-d case by Castro and Zuazua [6], and that in the 1-d case, the counter-example of Avellaneda et al. [1] shows that (0.10) fails for $\gamma_{0}$ large. Indeed, in the 1-d case, when $\rho=\rho(x / \varepsilon)$, Castro [5] has shown that the greatest value of $\gamma_{0}$ such that (0.10) holds true for some $T_{0}$ (when $V \Subset[a, b]=\Omega$ ) is related with the first instability interval of the Hill equation on the line $\left(\frac{d}{d y}\right)^{2}+\omega^{2} \rho(y)$. In the multi-d case, the understanding of the best value of $\gamma_{0}$ such that $(0.10)$ holds true will clearly involve the understanding of the localization and propagation of Bloch waves for the boundary value problem (0.4): this highly difficult problem is out of the scope of the present paper.

The conserved energy for solutions of $(0.4)$ is

$$
E\left(u^{\varepsilon}\right)=\frac{1}{2} \int_{\Omega}\left\{\left|\partial_{t} u^{\varepsilon}\right|^{2} \rho(x, x / \varepsilon)+\left|\nabla_{g} u^{\varepsilon}\right|^{2}\right\} d_{g} x .
$$

Applying the estimate $(0.10)$ to $\partial_{t} u^{\varepsilon}$, one easily gets the energy observability estimate

Corollary 0.1. Under the hypothesis and with the notations of Theorem 0.1, there exists a constant $C_{0}$ s.t. for any $\varepsilon \in] 0, \varepsilon_{0}\left[\right.$ and any $u^{\varepsilon} \in J_{\gamma_{0}}^{\varepsilon}$ one has

$$
E\left(u^{\varepsilon}\right) \leq C_{0} \int_{0}^{T_{0}} \int_{V}\left|\partial_{t} u^{\varepsilon}\right|^{2} \rho(x, x / \varepsilon) d t d_{g} x .
$$

The paper is organized as follows:

1. reduction to a semi-classical estimate;

2. the Bloch wave;

3. Lopatinski estimate;

4. propagation estimate;

5. Appendix A: semi-classical o.p.d with operators values;

6. Appendix B: proofs of Lemmas 3.4-3.6.

1. In the first part, using a Littlewood-Paley decomposition, we reduce the proof of the inequality (0.10) to the assertion

$$
\left\{\begin{array}{l}
\text { there exist } \left.\gamma_{0}, \varepsilon_{0}, h_{0}, C_{0} \text { such that for any } \varepsilon \in\right] 0, \varepsilon_{0}[\text {, and } \\
h \in\left[\varepsilon / \gamma_{0}, h_{0}\right] \text { the inequality }(0.10) \text { holds true for any } u^{\varepsilon} \in I_{h}^{\varepsilon}, \\
\text { where } I_{h}^{\varepsilon}=\left\{u^{\varepsilon}=\sum_{0.9 \leq \omega_{n}^{\varepsilon} h \leq 2.1}\left(u_{+, n} e^{i t \omega_{n}^{\varepsilon}}+u_{-, n} e^{-i t \omega_{n}^{\varepsilon}}\right) e_{n}^{\varepsilon}(x)\right\} .
\end{array}\right.
$$

2. In the second part, we introduce the Bloch wave at the boundary $\Gamma\left(u^{\varepsilon}\right)$. We refer to [2] and [7] for the study of Bloch waves in equations with oscillating coefficients. We choose a coordinate system

$$
\left\{\begin{array}{l}
\partial \Omega \times\left[0, r_{0}\right] \stackrel{\Theta}{\longrightarrow} \mathbb{R}^{d} \\
\left(x^{\prime}, x_{d}\right) \mapsto \Theta\left(x^{\prime}, x_{d}\right)
\end{array}\right.
$$

which satisfies

$$
\left\{\begin{array}{l}
\text { i) } \Theta\left(\partial \Omega \times\left[0, r_{0}\right]\right) \subset \bar{\Omega} \\
\text { ii) for } x_{d} \text { small }, x_{d} \mapsto \Theta\left(x^{\prime}, x_{d}\right) \text { is the geodesic normal to the } \\
\text { boundary at } x^{\prime} \in \partial \Omega, \text { for the metric } g \text { on } \bar{\Omega} \text {. }
\end{array}\right.
$$


In these coordinates, the Laplace operator takes the form

$$
\left\{\begin{array}{c}
\Delta_{g}=\frac{\partial}{\partial x_{d}}\left(A_{0}(x) \frac{\partial}{\partial x_{d}}+A_{1}\left(x, \partial_{x^{\prime}}\right)\right)+A_{2}\left(x, \partial_{x^{\prime}}\right) ; \\
x=\left(x^{\prime}, x_{d}\right), x^{\prime} \in \partial \Omega
\end{array}\right.
$$

where $A_{j}\left(x, \partial_{x^{\prime}}\right)$ are differential operators of order $j$ on $\partial \Omega$, with $x_{d}$ as parameter. Let $a_{j}\left(x, \xi^{\prime}\right)$ be the principal symbol of $A_{j}$. The dual metric $g^{-1}(x, \xi) \stackrel{\text { def }}{=}\|\xi\|_{x}^{2}$ on the cotangent bundle $T^{*} \Omega$ is

$$
\|\xi\|_{x}^{2}=a_{0}(x) \xi_{d}^{2}+a_{1}\left(x, \xi^{\prime}\right) \xi_{d}+a_{2}\left(x, \xi^{\prime}\right) .
$$

Let $\mathbb{T}^{d}=(\mathbb{R} / 2 \pi \mathbb{Z})^{d}$ be the $d$-dimensional torus and for $\varepsilon>0, S_{\varepsilon} \subset \partial \Omega \times\left[0, r_{0}\right] \times \mathbb{T}_{y}^{d}$ the submanifold

$$
S_{\varepsilon}=\left\{(x, y) ; y=\Theta(x) / \varepsilon \bmod (2 \pi \mathbb{Z})^{d}\right\} .
$$

Let $f(x)$ be a function on $\partial \Omega \times\left[0, r_{0}\right]$. We define a distribution $T(f)$ on $\partial \Omega \times\left[0, r_{0}\right] \times \mathbb{T}_{y}^{d}$ by the formula

$$
T(f)=\sum_{\ell \in \mathbb{Z}^{d}} e^{i \ell(y-\Theta(x) / \varepsilon)} f(x)=(2 \pi)^{d} \delta_{y=\Theta(x) / \varepsilon} \otimes f(x) .
$$

If $X$ is a vector field on $\partial \Omega \times\left[0, r_{0}\right]$, we shall denote by $X_{\varepsilon}^{*}$ the lift of $X$ on $S_{\varepsilon}$. If $x^{\prime}=\left(x_{1}, \ldots, x_{d-1}\right)$ is a local coordinate system on $\partial \Omega$, and $\left(\Theta_{1}(x), \ldots, \Theta_{d}(x)\right)=\Theta(x)$ are the Cartesian coordinates of $\Theta$, one has

$$
\left(\frac{\partial}{\partial x_{k}}\right)_{\varepsilon}^{*}=\frac{\partial}{\partial x_{k}}+\frac{1}{\varepsilon} \sum_{j=1}^{d} \frac{\partial \Theta_{j}}{\partial x_{k}}(x) \frac{\partial}{\partial y_{j}} \text { for } 1 \leq k \leq d
$$

and

$$
\left(\frac{\partial}{\partial x_{k}}\right)_{\varepsilon}^{*} T(f)=T\left(\frac{\partial}{\partial x_{k}} f\right) \text { for } 1 \leq k \leq d
$$

The Bloch operator on $\partial \Omega \times\left[0, r_{0}\right] \times \mathbb{T}^{d}$ is defined by

$$
\left\{\begin{array}{l}
\mathbb{B}_{\varepsilon}\left(x, \varepsilon \partial_{x}, \varepsilon \partial_{t} ; y, \partial_{y}\right)=\hat{\rho}(x, y)\left(\varepsilon \partial_{t}\right)^{2}-\varepsilon^{2}\left(\Delta_{g}\right)_{\varepsilon}^{*} ; \hat{\rho}(x, y)=\rho(\Theta(x), y) \\
\left(\Delta_{g}\right)_{\varepsilon}^{*}=\left(\frac{\partial}{\partial x_{d}}\right)_{\varepsilon}^{*}\left(A_{0}(x)\left(\frac{\partial}{\partial x_{d}}\right)_{\varepsilon}^{*}+A_{1}\left(x,\left(\partial_{x^{\prime}}\right)\right)_{\varepsilon}^{*}\right)+A_{2}\left(x,\left(\partial_{x^{\prime}}\right)_{\varepsilon}^{*}\right) .
\end{array}\right.
$$

It satisfies the identity

$$
\mathbb{B}_{\varepsilon}(T(u(x, t)))=\varepsilon^{2} T\left(\left(\rho(\Theta(x), \Theta(x) / \varepsilon) \partial_{t}^{2}-\Delta_{g}\right)(u(x, t))\right) .
$$

Let $\widetilde{A}_{j}$ be the operators

$$
\widetilde{A}_{j}=A_{j}\left(x,\left(\partial_{x^{\prime}}\right)_{\varepsilon}^{*}\right)
$$

and let $e_{k}(x) 1 \leq k \leq d$ be the vectors of $\mathbb{R}^{d}$

$$
e_{k}(x)=\frac{\partial \Theta}{\partial x_{k}}(x)
$$


If $v(t, x, y)$ is a distribution on $\stackrel{\circ}{X} \times \mathbb{T}^{d}$, with $X=\mathbb{R}_{t} \times\left(\partial \Omega \times\left[0, r_{0}\right]\right)$, we shall write the equation $\mathbb{B}_{\varepsilon}(v)=0$ as a $2 \times 2$ system for the vector $w=\mathcal{A}(v)$.

$$
\mathcal{A}(v)=\left[\begin{array}{l}
w_{0} \\
w_{1}
\end{array}\right] \stackrel{\text { def }}{=}\left[\begin{array}{l}
v \\
\left(A_{0}(x)\left(\varepsilon \frac{\partial}{\partial x_{d}}\right)_{\varepsilon}^{*}+\varepsilon \widetilde{A}_{1}\right) v
\end{array}\right] .
$$

This system takes the form

$$
\left\{\begin{array}{l}
\varepsilon \frac{\partial}{\partial x_{d}} w+\mathbb{M} w=0 \\
\mathbb{M}=\left[\begin{array}{cc}
e_{d}(x) \cdot \partial_{y}+\varepsilon A_{0}^{-1}(x) \widetilde{A}_{1} & -A_{0}^{-1}(x) \\
\varepsilon^{2} \widetilde{A}_{2}-\hat{\rho}(x, y)\left(\varepsilon \partial_{t}\right)^{2} & e_{d}(x) \cdot \partial_{y}
\end{array}\right]
\end{array}\right.
$$

The operator $\mathbb{M}$ will be seen as a semi-classical operator in $t, x, \frac{\varepsilon}{i} \partial_{x^{\prime}}=\xi^{\prime}, \frac{\varepsilon}{i} \partial_{t}=\tau$ with operator values in the fiber $\mathbb{T}^{d}$

$$
\mathbb{M}=\sum_{j=0}^{2}\left(\frac{\varepsilon}{i}\right)^{j} \mathbb{M}^{j}\left(x, \xi^{\prime}, \tau ; y, \partial_{y}\right) .
$$

The differential degree in $y$ of $\mathbb{M}^{j}$ is at most $2-j$ and the principal symbol $\mathbb{M}^{0}$ is the matrix

$$
\mathbb{M}^{0}\left(x, \xi^{\prime}, \tau ; y, \partial_{y}\right)=\left[\begin{array}{ll}
e_{d}(x) \cdot \partial_{y}+a_{0}^{-1}(x) a_{1}\left(x, i \xi^{\prime}+e^{\prime}(x) \cdot \partial_{y}\right) & -a_{0}^{-1}(x) \\
a_{2}\left(x, i \xi^{\prime}+e^{\prime}(x) \cdot \partial_{y}\right)+\hat{\rho}(x, y) \tau^{2} & e_{d}(x) \cdot \partial_{y}
\end{array}\right]
$$

Let $E^{\bullet}=\left\{E^{s}, s \in \mathbb{R}\right\}$ be the scale of Hilbert spaces on the torus

$$
E^{s}=H^{s}\left(\mathbb{T}^{d}\right) \oplus H^{s-1}\left(\mathbb{T}^{d}\right)
$$

For any $\rho=\left(x, \xi^{\prime}, \tau\right), \mathbb{M}^{j}\left(\rho, y, \partial_{y}\right)$ maps $E^{s}$ into $E^{s-1+j}$ and $\mathbb{M}^{0}$ is an elliptic operator. Let $\mathbb{M}_{0}^{0}$ be the restriction of $\mathbb{M}^{0}$ to the zero section $\xi^{\prime}=\tau=0$.

$$
\mathbb{M}_{0}^{0}\left(x, \partial_{y}\right)=\mathbb{M}^{0}\left(x, 0,0, y, \partial_{y}\right)=\left[\begin{array}{ll}
e_{d}(x) \cdot \partial_{y}+a_{0}^{-1} a_{1}\left(x, e^{\prime}(x) \cdot \partial_{y}\right) & -a_{0}^{-1}(x) \\
a_{2}\left(x, e^{\prime}(x) \cdot \partial_{y}\right) & e_{d}(x) \cdot \partial_{y}
\end{array}\right]
$$

The eigenvalues $\lambda_{ \pm, \ell}^{0}(x)$ of $\frac{1}{i} \mathbb{M}_{0}^{0}\left(x, \partial_{y}\right)$ on the space $e^{i \ell y} \mathbb{C}^{2}$, for $\ell \in \mathbb{Z}^{d}$ are the complex roots of the equation

$$
a_{0}(x)\left(-\lambda+e_{d} . \ell\right)^{2}+\left(-\lambda+e_{d} \cdot \ell\right) a_{1}\left(x, e^{\prime} . \ell\right)+a_{2}\left(x, e^{\prime} \cdot \ell\right)=0
$$

which is equivalent to

$$
\left\|^{t} d \Theta(x)(\ell)-\lambda(0, \cdots, 0,1)\right\|_{x}^{2}=0 .
$$

In particular we have

$$
\inf _{x} \min _{\ell \neq 0}\left|\lambda_{ \pm, \ell}^{0}(x)\right|>0
$$

so the double eigenvalue $\lambda_{ \pm, 0}^{0}(x)=0$ is isolated in the spectrum of $\mathbb{M}_{0}^{0}\left(x, \partial_{y}\right)$.

In the sequel, we shall restrict the values of the Sobolev index of regularity $s$ on the torus to some fixed large interval, $s \in\left[-\sigma_{0}, \sigma_{0}\right], \sigma_{0} \gg \frac{d}{2}$. 
Let $X=\partial \Omega \times \mathbb{R}_{t} \times\left[0, r_{0}\right]$. We denote by ${ }^{t} T^{*} X$ the tangential cotangent bundle

$$
{ }^{t} T^{*} X=T^{*}\left(\partial \Omega \times \mathbb{R}_{t}\right) \times\left[0, r_{0}\right] .
$$

Let $W_{1} \Subset W_{0}$ be two small neighborhoods of the set $\left\{\xi^{\prime}=\tau=0\right\} \times\left\{t \in\left[-T_{0}, 2 T_{0}\right]\right\}$ in ${ }^{t} T^{*} X$.

We choose a non-negative function $\chi_{0} \in C_{0}^{\infty}\left(W_{0}\right)$, such that $\chi_{0} \equiv 1$ on $W_{1}$.

If $W_{0}$ is small enough, we define the map $p_{0}\left(x, t, \xi^{\prime}, \tau\right): E^{\bullet} \rightarrow \mathbb{C}^{2}$ by the formula

$$
p_{0}[w]=\chi_{0} \cdot \oint_{\mathbb{T}^{d}}\left\{\frac{1}{2 i \pi} \int_{\partial D} \frac{d z}{z-\mathbb{M}^{0}}\right\}[w] \quad w \in E^{s}, s \in\left[-\sigma_{0}, \sigma_{0}\right]
$$

(where $D \subset \mathbb{C}$ is a small disk with center $z=0$ ).

It satisfies the estimates

$$
\exists C \forall s \in\left[-\sigma_{0}, \sigma_{0}\right] \forall w \in E^{s} \quad\left\|p_{0}(w)-\chi_{0} \oint_{\mathbb{T}^{d}} w\right\|_{\mathbb{C}^{2}} \leq C \tau^{2}\|w\|_{E^{s}}
$$

and there exists $L^{0}\left(x, t, \xi^{\prime}, \tau\right) \in C^{\infty}\left({ }^{t} T^{*} X ; M_{2}(\mathbb{C})\right)$, defined near $\xi^{\prime}=\tau=0$ such that (see (2.29-2.31))

$$
p_{0} \circ \mathbb{M}^{0}=L^{0} \circ p_{0} .
$$

By a Taylor expansion near $\xi^{\prime}=\tau=0$, one gets

$$
L^{0}=\left[\begin{array}{cc}
a_{0}^{-1}(x) a_{1}\left(x, i \xi^{\prime}\right) & -a_{0}^{-1}(x) \\
a_{2}\left(x, i \xi^{\prime}\right)+\underline{\hat{\rho}}(x) \tau^{2} & 0
\end{array}\right]+O\left(\tau^{4}\right) .
$$

We then suitably quantize the above construction and we obtain tangential pseudo differential operators (see Append. A1)

$$
\begin{cases}\Pi_{0}\left(\varepsilon, t, x, \varepsilon \partial_{t}, \varepsilon \partial_{x^{\prime}}\right) & : L^{2}\left(X ; E^{s}\right) \rightarrow L^{2}\left(X, \mathbb{C}^{2}\right), s \in\left[-\sigma_{0}, \sigma_{0}\right] \\ L\left(\varepsilon, t, x, \varepsilon \partial_{t}, \varepsilon \partial_{x^{\prime}}\right) & : L^{2}\left(X ; \mathbb{C}^{2}\right) \rightarrow L^{2}\left(X, \mathbb{C}^{2}\right)\end{cases}
$$

with principal symbol $\sigma\left(\Pi_{0}\right)=p_{0}, \sigma(L)=L^{0}$, which satisfy the relation

$$
\Pi_{0}\left(\varepsilon \partial_{x_{d}}+\mathbb{M}\right)=\left(\varepsilon \partial_{x_{d}}+L\right) \Pi_{0}+R\left(\varepsilon, t, x, \varepsilon \partial_{t}, \varepsilon \partial_{x^{\prime}}\right) .
$$

In (0.41), the error term $R: L^{2}\left(X ; E^{s}\right) \rightarrow L^{2}\left(X, \mathbb{C}^{2}\right)$ will be a tangential pseudo differential operator such that for any tangential o.p.d. $Q$ with essential support in $W_{1}$ and any $s \in\left[-\sigma_{0}, \sigma_{0}\right]$, one has

$$
\left\|Q \circ R ; L^{2}\left(X ; E^{s}\right) \rightarrow L^{2}\left(X, \mathbb{C}^{2}\right)\right\| \in . O\left(\varepsilon^{\infty}\right) .
$$

Definition 0.1. For $u^{\varepsilon} \in I_{h}^{\varepsilon}$, we define the Bloch wave $\Gamma\left(u^{\varepsilon}\right) \in L^{2}\left(X ; \mathbb{C}^{2}\right)$ by the formula

$$
\Gamma\left(u^{\varepsilon}\right)=\left[\begin{array}{l}
\Gamma_{0}\left(u^{\varepsilon}\right) \\
\Gamma_{1}\left(u^{\varepsilon}\right)
\end{array}\right]=\Pi_{0} \mathcal{T}\left(u^{\varepsilon}\right) \quad(\mathcal{T}=\mathcal{A} \circ T) .
$$

Let $\gamma_{0}, \varepsilon_{0}, h_{0}$ be given small enough, $\left.\left.\varepsilon \in\right] 0, \varepsilon_{0}\right], h \in\left[\varepsilon / \gamma_{0}, h_{0}\right]$. For $u_{\varepsilon} \in I_{h}^{\varepsilon}, u^{\varepsilon}=\sum_{0.9 \leq \omega_{n}^{\varepsilon} h \leq 2.1}\left(u_{+, n} e^{i t \omega_{n}^{\varepsilon}}\right.$ $\left.+u_{-, n} e^{-i t \omega_{n}^{\varepsilon}}\right) e_{n}^{\varepsilon}(x)$, we define $\left\|u^{\varepsilon}\right\|^{2}\left(\simeq \int_{0}^{T_{0}} \int_{\Omega}\left|u^{\varepsilon}\right|^{2}\right)$ by

$$
\left\|u^{\varepsilon}\right\|^{2}=\sum_{0.9 \leq \omega_{n}^{\varepsilon} h \leq 2.1}\left|u_{+, n}\right|^{2}+\left|u_{-, n}\right|^{2} .
$$


Let $X_{T_{0}}=\partial \Omega \times\left[-T_{0}, 2 T_{0}\right] \times\left[0, r_{0}\right]$, and let $K$ be the compact subset of ${ }^{t} T^{*} X, K=\partial \Omega \times\left[0, T_{0}\right] \times\left[0, r_{0} / 2\right]$ $\times\left\{\xi^{\prime}=0, \tau=0\right\}$. The following proposition will be proven in Section 2 .

Proposition 0.1. Let $Q\left(\varepsilon, t, x, \varepsilon \partial_{x^{\prime}}, \varepsilon \partial_{t}\right)$ be a zero order tangential opd on $X$, equal to Id near $K$. If $\gamma_{0}, \varepsilon_{0}, h_{0}$ are small enough, there exists a constant $C>0$, such that for any $\left.\varepsilon \in] 0, \varepsilon_{0}\right], h \in\left[\varepsilon / \gamma_{0}, h_{0}\right]$, one has

$$
\left\|u^{\varepsilon}\right\|^{2} \leq C\left[\left\|Q \Gamma_{0}\left(u^{\varepsilon}\right)\right\|_{L^{2}\left(X_{T_{0}}\right)}^{2}+\left\|u^{\varepsilon}\right\|_{L^{2}\left(\left(0, T_{0}\right) \times V\right)}^{2}\right] \forall u^{\varepsilon} \in I_{h}^{\varepsilon}
$$

3. By Proposition 0.1, we shall obtain the inequality (0.10), if we are able to estimate the $L^{2}$ norm of the first component $\Gamma_{0}\left(u^{\varepsilon}\right)$ of the Bloch wave near the set $K$.

The formula (0.41) shows that $\Gamma\left(u^{\varepsilon}\right)$ satisfies the equation

$$
\left.\left(\varepsilon \partial_{x_{d}}+L\right) \Gamma\left(u^{\varepsilon}\right) \in O\left(\varepsilon^{\infty} L^{2}\right) \text { (microlocally in } W_{1}\right) .
$$

By $(0.39)$ this equation is very closed to the homogenized equation $\left(\underline{\rho}(x) \partial_{t}^{2}-\Delta_{g}\right)\left[\Gamma_{0}\left(u^{\varepsilon}\right)\right]=0$.

As one can see, all the difficulty in our problem is thus to obtain an estimate on the first Dirichlet data of $\Gamma\left(u^{\varepsilon}\right)$ on the boundary $x_{d}=0$, in order to apply propagation arguments to the equation $(0.46)$. We shall prove the following proposition.

Proposition 0.2. If $\gamma_{0}, \varepsilon_{0}, h_{0}$ are small enough, there exists a constant $C$ such that for any $\left.\varepsilon \in\right] 0, \varepsilon_{0}[$, $h \in\left[\varepsilon / \gamma_{0}, h_{0}\right]$ the following estimate holds true

$$
\left\|\Gamma_{0}\left(u^{\varepsilon}\right)_{\mid x_{d}=0}\right\|_{L^{2}\left(X_{T_{0}} \cap x_{d}=0\right)} \leq C \varepsilon / h\left\|u^{\varepsilon}\right\| \quad \forall u^{\varepsilon} \in I_{h}^{\varepsilon} .
$$

The above estimate will be obtained as a consequence of a uniform Lopatinski estimate on $w^{\varepsilon}=\mathcal{T}\left(u^{\varepsilon}\right)=\left[\begin{array}{l}w_{0}^{\varepsilon} \\ w_{1}^{\varepsilon}\end{array}\right]$. We shall prove

Theorem 0.2. Let $Q$ be a scalar tangential o.p.d. with essential support in $W_{0}$; if $W_{0}, \gamma_{0}, \varepsilon_{0}, h_{0}$ are small enough, there exist $s_{1}<0$ and a constant $C$ such that for any $u^{\varepsilon} \in I_{h}^{\varepsilon}$ the following estimate holds true

$$
\left\|Q\left(t, x, \varepsilon \partial_{x^{\prime}}, \varepsilon \partial_{t}\right)\left(w_{1}^{\varepsilon}\right)_{\mid x_{d}=0}\right\|_{L^{2}\left(X_{T_{0}} \cap x_{d}=0, H^{s_{1}}\left(\mathbb{T}^{d}\right)\right)} \leq C\left\|u^{\varepsilon}\right\|
$$

Notice that $w^{\varepsilon}$ satisfies the equation $(0.27)$, with Dirichlet data $w_{0 \mid x_{d}=0}^{\varepsilon}=0$ on the boundary.

The weaker estimate

$$
\left\|Q\left(w_{1}^{\varepsilon}\right)_{\mid x_{d}=0}\right\| \leq C \varepsilon^{-1 / 2}\left\|u^{\varepsilon}\right\|
$$

is easy to obtain (it is sufficient to commute the Eq. (0.4) with the normal vector field $\frac{\partial}{\partial n}$ ).

The proof of (0.48) is the most technical part of our work. It involves a detailed study of how the spectral theory of $\mathbb{M}^{0}\left(x, \xi^{\prime}, \tau ; y, \partial y\right)$ (see $\left.(0.29)\right)$ depends on the parameter $\left(x, \xi^{\prime}, \tau\right)$.

4. This part will be devoted to the proof of the following proposition.

Proposition 0.3. Let $Q\left(\varepsilon, t, x, \varepsilon \partial_{x^{\prime}}, \varepsilon \partial_{t}\right)$ be a zero order opd equal to Id near $K$, with essential support in $W_{1}$. There exist $\gamma_{0}, \varepsilon_{0}, h_{0}$, and a constant $C_{0}$ such that, for any $\left.\left.\varepsilon \in\right] 0, \varepsilon_{0}\right], h \in\left[\varepsilon / \gamma_{0}, h_{0}\right]$ and $u^{\varepsilon} \in I_{h}^{\varepsilon}$, the following estimate holds true 


$$
\left\|Q \Gamma_{0}\left(u^{\varepsilon}\right)\right\|_{L^{2}\left(X_{T_{0}}\right)}^{2} \leq C_{0}\left[\left\|\Gamma_{0}\left(u^{\varepsilon}\right)_{\mid x_{d}=0}\right\|_{L^{2}\left(X_{T_{0}} \cap x_{d}=0\right)}^{2}+\left\|u^{\varepsilon}\right\|_{L^{2}\left(0, T_{0}\right) \times V}^{2}\right] .
$$

This estimate will be obtained by rather classical arguments in the theory of control of linear waves, for the rescale equation

$$
\begin{cases}\left(h \frac{\partial}{\partial x_{d}}+\mathcal{L}\right)\left[\begin{array}{l}
g_{0} \\
g_{1}
\end{array}\right] \sim 0 & {\left[\begin{array}{l}
g_{0} \\
g_{1}
\end{array}\right]=\left[\begin{array}{c}
\Gamma_{0}\left(u^{\varepsilon}\right) \\
\frac{h}{\varepsilon} \Gamma_{1}\left(u^{\varepsilon}\right)
\end{array}\right]} \\
\mathcal{L}=\frac{h}{\varepsilon}\left(\begin{array}{cc}
1 & 0 \\
0 & h / \varepsilon
\end{array}\right) \circ L \circ\left(\begin{array}{cc}
1 & 0 \\
0 & \varepsilon / h
\end{array}\right) . & \end{cases}
$$

We shall verify that $\mathcal{L}$ is still a $h$-pseudo differential operator, with $\varepsilon / h$ as parameter. (We use this rescaling in order to be able to use propagation arguments in the range $\varepsilon \ll h$.)

5. In Appendix A.1, we recall the properties of the semi-classical calculus with operators values which is used in 2. In Appendix A.2, we extend this calculus to a larger class of symbols; this exotic calculus will be used in 3.

To end this introduction, we finally remark that the validity of (0.13), hence the proof of Theorem 0.1 , is a direct consequence of the Propositions 0.1, 0.2 and 0.3.

\section{Semi-Classical Reduction}

In this part, we verify that (0.13) implies the Theorem 1.

Let $e_{n}^{\varepsilon}(x)$ be a normalized eigenfunction of the Dirichlet problem (0.3), and let $\mu_{1}$ be the first eigenvalue of the Dirichlet problem for $\Delta_{g}$ in $\Omega$. One has

$$
\int_{\Omega}\left|\nabla_{g} e_{n}^{\varepsilon}\right|^{2} d_{g} x=\int_{\Omega} \rho(x, x / \varepsilon)\left(\omega_{n}^{\varepsilon}\right)^{2}\left|e_{n}^{\varepsilon}(x)\right|^{2} d_{g} x \leq \rho_{\max }\left(\omega_{n}^{\varepsilon}\right)^{2} \int_{\Omega}\left|e_{n}^{\varepsilon}(x)\right|^{2} d_{g} x .
$$

So we get the uniform lower bound

$$
\omega_{n}^{\varepsilon} \geq\left(\rho_{\max }\right)^{-1 / 2} \mu_{1}^{1 / 2} .
$$

The Sobolev spaces $L^{2}(\Omega), H_{0}^{1}(\Omega), H^{-1}(\Omega)$, with norms $\left(\int_{\Omega}|f|^{2} \rho d_{g} x\right)^{1 / 2},\left(\int_{\Omega}\left|\nabla_{g} f\right|^{2} d_{g} x\right)^{1 / 2}, \sup \left\{\int_{\Omega} f \bar{h} \rho d_{g} x\right.$, $\left.\|h\|_{H_{0}^{1}} \leq 1\right\}$ are characterized in terms of Fourier series by

$$
\left\{\begin{array}{l}
f_{n}^{\varepsilon}=\int_{\Omega} f \overline{e_{n}^{\varepsilon}(x)} \rho d_{g} x \text { for } f \in H^{-1}(\Omega) \\
\|f\|_{L^{2}}^{2}=\sum_{n}\left|f_{n}^{\varepsilon}\right|^{2} ;\|f\|_{H_{0}^{1}}^{2}=\sum_{n}\left(\omega_{n}^{\varepsilon}\right)^{2}\left|f_{n}^{\varepsilon}\right|^{2} ;\|f\|_{H^{-1}}^{2}=\sum_{n}\left(\omega_{n}^{\varepsilon}\right)^{-2}\left|f_{n}^{\varepsilon}\right|^{2} .
\end{array}\right.
$$

Any solution $u^{\varepsilon}$ of the wave equation (0.4) with data $\left(u^{\varepsilon}(0), \partial_{t} u^{\varepsilon}(0)\right) \in L^{2}(\Omega) \oplus H^{-1}(\Omega)$ is of the form

$$
u^{\varepsilon}=\sum_{n} u_{n}^{\varepsilon}(t) e_{n}^{\varepsilon}(x)=\sum_{n}\left(u_{+, n}^{\varepsilon} e^{i t \omega_{n}^{\varepsilon}}+u_{-, n}^{\varepsilon} e^{-i t \omega_{n}^{\varepsilon}}\right) e_{n}^{\varepsilon}(x)
$$

with $\left(u_{ \pm, n}^{\varepsilon}\right)_{n} \in \ell^{2}$, and (1.2) implies that there exists a constant $C$ independent of $\varepsilon$, s.t.

$$
\frac{1}{C} \sum_{n, \pm}\left|u_{ \pm, n}^{\varepsilon}\right|^{2} \leq \int_{0}^{T_{0}} \int_{\Omega}\left|u^{\varepsilon}\right|^{2} \rho d t d_{g} x \leq C \sum_{n, \pm}\left|u_{ \pm, n}^{\varepsilon}\right|^{2} .
$$


If the geometric hypothesis (0.9) holds true for $T_{0}$, it remains valid for $T_{0}-2 \delta$, for $\delta>0$ small enough; we can therefore assume that $(0.13)$ is valid on $\left[\delta, T_{0}-\delta\right]$.

Take $\varphi(t) \in C_{0}^{\infty}(] 0, T_{0}[), \varphi(t) \equiv 1$ on $\left[\delta, T_{0}-\delta\right]$ and $\psi(\sigma) \in C_{0}^{\infty}(] 0.9,2.1[), \psi(\sigma) \equiv 1$ on $[1,2]$. Let $\chi(\sigma)$ $=\psi(\sigma)+\psi(-\sigma)$. For $u^{\varepsilon} \in J_{\gamma_{0}}^{\varepsilon}$, one has $\chi\left(2^{-k} D_{t}\right) u^{\varepsilon} \in I_{2^{-k}}^{\varepsilon}$, so there exists $C_{0}$ s.t.

$$
\left\{\begin{array}{l}
\left.\forall \varepsilon \in] 0, \varepsilon_{0}\right], \forall k \in \mathbb{N} \text { s.t. } 2^{-k} \in\left[\varepsilon / \gamma_{0}, h_{0}\right] \\
\forall u^{\varepsilon}=\sum_{\varepsilon \omega_{n}^{\varepsilon} \leq \gamma_{0}}\left(u_{+, n}^{\varepsilon} e^{i t \omega_{n}}+u_{-, n}^{\varepsilon} e^{-i t \omega_{n}}\right) e_{n}^{\varepsilon}(x) \in J_{\gamma_{0}}^{\varepsilon} \\
\quad \sum_{2^{k} \leq \omega_{n}^{\varepsilon} \leq 2^{k+1}}\left|u_{+, n}^{\varepsilon}\right|^{2}+\left|u_{-, n}^{\varepsilon}\right|^{2} \leq C_{0} \int_{-\infty}^{+\infty} d t \int_{V} d_{g} x\left|\varphi(t) \chi\left(2^{-k} D_{t}\right) u^{\varepsilon}\right|^{2} .
\end{array}\right.
$$

On the other hand,using classical estimates as in ([9], Sect. 4), one gets $\exists C_{1}, C_{2}, k_{0}$ s.t. for any $k_{1} \geq k_{0}$, and any $u^{\varepsilon} \in J_{\gamma_{0}}^{\varepsilon}$

$$
\sum_{k \geq k_{1}} \int_{-\infty}^{+\infty} d t \int_{V}\left|\varphi(t) \chi\left(2^{-k} D_{t}\right) u^{\varepsilon}\right|^{2} d_{g} x \leq C_{1} \int_{0}^{T_{0}} \int_{V}\left|u^{\varepsilon}\right|^{2} d_{g} x+C_{2} 2^{-2 k_{1}}\left(\sum_{n}\left|u_{ \pm, n}^{\varepsilon}\right|^{2}\right) .
$$

Let $\gamma_{1}=\gamma_{0} / 2$; for $u^{\varepsilon} \in J_{\gamma_{1}}^{\varepsilon}$ and $2^{-k}<\varepsilon / \gamma_{0}$ one has $\chi\left(2^{-k} D_{t}\right) u^{\varepsilon} \equiv 0$, so putting together (1.6) and (1.7) we get

$$
\left\{\begin{array}{l}
\left.\left.\exists n_{0}, \exists C_{3}, \forall \varepsilon \in\right] 0, \varepsilon_{0}\right], \forall u^{\varepsilon} \in J_{\gamma_{1}}^{\varepsilon} \\
\sum_{n \geq n_{0}, \varepsilon \omega_{n}^{\varepsilon} \leq \gamma_{1}}\left|u_{+, n}^{\varepsilon}\right|^{2}+\left|u_{-, n}^{\varepsilon}\right|^{2} \leq C_{3}\left(\int_{0}^{T_{0}} d t \int_{V} d_{g} x\left|u^{\varepsilon}\right|^{2}+\sum_{n \leq n_{0}}\left|u_{ \pm, n}^{\varepsilon}\right|^{2}\right)
\end{array}\right.
$$

and (1.8) is equivalent to

$$
\left\{\begin{array}{l}
\left.\left.\exists n_{0}, \exists C_{4}, C_{5}, \forall \varepsilon \in\right] 0, \varepsilon_{0}\right], \forall u^{\varepsilon} \in J_{\gamma_{1}}^{\varepsilon} \\
\int_{0}^{T} \int_{\Omega}\left|u^{\varepsilon}\right|^{2} \rho d t d_{g} x \leq C_{3} \int_{0}^{T_{0}} \int_{V}\left|u^{\varepsilon}\right|^{2} \rho d t d_{g} x \\
+C_{4}\left(\sum_{n \leq n_{0}}\left|u_{+, n}^{\varepsilon}\right|^{2}+\left|u_{-, n}^{\varepsilon}\right|^{2}\right) .
\end{array}\right.
$$

It is now easy to conclude the proof of Theorem 1 by a uniqueness argument. In fact if (0.10) is untrue, there exist a sequence $\varepsilon_{k} \rightarrow 0$ and $u^{\varepsilon_{k}} \in J_{\gamma_{1}}^{\varepsilon_{k}}$ such that $\int_{0}^{T_{0}} \int_{\Omega}\left|u^{\varepsilon_{k}}\right|^{2} \rho d t d_{g} x=1$ and $\int_{0}^{T_{0}} \int_{V}\left|u^{\varepsilon_{k}}\right|^{2} \rho d t d_{g} x \rightarrow 0$; let $u$ be a weak limit in $L^{2}$ of $\left\{u^{\varepsilon_{k}}\right\} ; u$ satisfies

$$
\left\{\begin{array}{l}
\underline{\rho}(x) \partial_{t}^{2} u-\Delta_{g} u=0 \text { on } \mathbb{R}_{t} \times \Omega \\
u_{\mid \partial \Omega}=0 ; u_{\mid] 0, T_{0}[\times V}=0
\end{array}\right.
$$

and from the observability inequality (0.7), we get $u \equiv 0$. Then (1.9) implies that $u \equiv 0$ is the strong limit in $L^{2}$ of $u^{\varepsilon_{k}}$, which contradicts $\int_{0}^{T_{0}} \int_{\Omega}\left|u^{\varepsilon_{k}}\right|^{2} \rho d t d_{g} x \equiv 1$.

\section{The Bloch WAVE}

We shall now recall how one can quantize the principal symbols maps $p_{0}, L^{0}$ defined in $(0.36,0.38)$ in order to obtain the pseudo differential relation (0.41). 
Let

$$
I=\left[-\sigma_{0}, \sigma_{0}\right]
$$

For any $s \in I$, we split $E^{s}=H^{s}\left(\mathbb{T}^{d}\right) \oplus H^{s-1}\left(\mathbb{T}^{d}\right)$ into the decomposition

$$
\left\{\begin{array}{lll}
E^{s} & =E_{0} \oplus E_{\perp}^{s} \quad E_{0}=\mathbb{C}^{2} \\
w & =w_{(0)}+w_{\perp} & w_{(0)}=\oint_{\mathbb{T}^{d}} w .
\end{array}\right.
$$

In other words we write $w=\sum_{\ell} w_{(\ell)} e^{i \ell y}$ and $w_{\perp}=\sum_{\ell \neq 0} w_{(\ell)} e^{i \ell y}$.

We then construct tangential pseudo differential operators defined near $\varepsilon \partial_{t}=i \tau=0$, $\varepsilon \partial_{x^{\prime}}=i \xi^{\prime}=0$, semi-classical in $\varepsilon$

$$
\begin{array}{rll}
A_{0}\left(\varepsilon, x, \varepsilon \partial_{t}, \varepsilon \partial_{x^{\prime}}\right) & : L^{2}\left(X, E_{0}\right) & \rightarrow L^{2}\left(X, \underset{s \in I}{\cap} E^{s}\right) \\
A_{\perp}\left(\varepsilon, x, \varepsilon \partial_{t}, \varepsilon \partial_{x^{\prime}}\right) & : L^{2}\left(X, E_{\perp}^{s}\right) & \rightarrow L^{2}\left(X, E^{s}\right) \quad(\forall s \in I) \\
L\left(\varepsilon, x, \varepsilon \partial_{t}, \varepsilon \partial_{x^{\prime}}\right) & : L^{2}\left(X, E_{0}\right) & \rightarrow L^{2}\left(X, E_{0}\right) \\
L_{\perp}\left(\varepsilon, x, \varepsilon \partial_{t}, \varepsilon \partial_{x^{\prime}}\right) & : L^{2}\left(X, E_{\perp}^{s}\right) & \rightarrow L^{2}\left(X, E_{\perp}^{s-1}\right) \quad(\forall s \in I)
\end{array}
$$

with symbols admitting asymptotic expansions

$$
\begin{array}{llll}
\sum_{k}\left(\frac{\varepsilon}{i}\right)^{k} A_{0}^{k}\left(x, \tau, \xi^{\prime}\right) & A_{0}^{k} \quad \text { bounded from } E_{0} \quad \text { to } \bigcap_{s \in I} E^{s} \\
\sum_{k}\left(\frac{\varepsilon}{i}\right)^{k} A_{\perp}^{k}\left(x, \tau, \xi^{\prime}\right) & A_{\perp}^{k} \text { bounded from } E_{\perp}^{s} \quad \text { to } E^{s} \quad(\forall s \in I) \\
\sum_{k}\left(\frac{\varepsilon}{i}\right)^{k} L^{k}\left(x, \tau, \xi^{\prime}\right) & L^{k} \text { bounded from } E_{0} \quad \text { to } E_{0} \\
\sum_{k}\left(\frac{\varepsilon}{i}\right)^{k} L_{\perp}^{k}\left(x, \tau, \xi^{\prime}\right) & L_{\perp}^{k} \text { bounded from } E_{\perp}^{s} \quad \text { to } E_{\perp}^{s-1}(\forall s \in I)
\end{array}
$$

such that near the zero section $\tau=\xi^{\prime}=0$, the two following identities hold true, in the algebra of tangential pseudo differential operators

$$
\left\{\begin{array}{l}
\left(\varepsilon \frac{\partial}{\partial_{x_{d}}}+\mathbb{M}\right) A_{0}=A_{0}\left(\varepsilon \partial_{x_{d}}+L\right) \\
\left(\varepsilon \frac{\partial}{\partial_{x_{d}}}+\mathbb{M}\right) A_{\perp}=A_{\perp}\left(\varepsilon \partial_{x_{d}}+L_{\perp}\right) .
\end{array}\right.
$$

Using the formula $(0.28) \mathbb{M}=\sum_{j=0}^{2}\left(\frac{\varepsilon}{i}\right)^{j} \mathbb{M}^{j}\left(x, \xi^{\prime}, \tau ; y, \partial y\right)$, and the rules of composition of pseudo differential operators, one gets that $(2.11)$ is equivalent to the following set of equations $(2.12,2.13)$

$$
k=0 \quad\left\{\begin{array}{l}
\mathbb{M}^{0} A_{0}^{0}=A_{0}^{0} L^{0} \\
\mathbb{M}^{0} A_{\perp}^{0}=A_{\perp}^{0} L_{\perp}^{0}
\end{array}\right.
$$




$$
\left\{\begin{array}{c}
\sum_{j+\ell+|\alpha|=k} \frac{1}{\alpha !} \partial_{\xi^{\prime}}^{\alpha} \mathbb{M}^{j} \partial_{x^{\prime}}^{\alpha} A_{0}^{\ell}+i \partial_{x_{d}} A_{0}^{k-1}=\sum_{j+\ell+|\alpha|=k} \frac{1}{\alpha !} \partial_{\xi^{\prime}}^{\alpha} A_{0}^{j} \partial_{x^{\prime}}^{\alpha} L^{\ell} \\
\sum_{j+\ell+|\alpha|=k} \frac{1}{\alpha !} \partial_{\xi^{\prime}}^{\alpha} \mathbb{M}^{j} \partial_{x^{\prime}}^{\alpha} A_{\perp}^{\ell}+i \partial_{x_{d}} A_{\perp}^{k-1}=\sum_{j+\ell+|\alpha|=k} \frac{1}{\alpha !} \partial_{\xi^{\prime}}^{\alpha} A_{\perp}^{k} \partial_{x^{\prime}}^{\alpha} L_{\perp}^{\ell}
\end{array}\right.
$$

Let $j_{0}$ and $j_{\perp}$ be the inclusion maps

$$
E_{0} \stackrel{j_{0}}{\hookrightarrow} E^{s} \quad E_{\perp}^{s} \stackrel{j_{\perp}}{\hookrightarrow} E^{s}
$$

and let $\pi_{0}=\pi_{0}\left(x, \xi^{\prime}, \tau\right)$ be the spectral projector of $\mathbb{M}^{0}$, which is defined near $\left(\xi^{\prime}, \tau\right)=(0,0)$, by

$$
\pi_{0}=\frac{1}{2 i \pi} \int_{\partial D} \frac{d z}{z-\mathbb{M}^{0}}
$$

where $D \subset \mathbb{C}$ is a small disk with center at $z=0$.

The range of $\pi_{0}$ is a two-dimensional invariant subspace of $\mathbb{M}^{0}$, and by the definition formula $(0.29)$ of $\mathbb{M}^{0}$, one gets for $|\tau|$ small enough

$$
\left\|\oint_{\mathbb{T}^{d}} \pi_{0} j_{0}-I d_{E_{0}}\right\| \leq \operatorname{Cte} \tau^{2} ;\left\|\pi_{0} j_{\perp} ; E_{\perp}^{s} \rightarrow E^{s}\right\| \leq \mathrm{Cte} \tau^{2} .
$$

In order to obtain the relations (2.12), it is clearly sufficient to select isomorphisms

$$
\begin{cases}A_{0}^{0}: E_{0} \rightleftharpoons \operatorname{range}\left(\pi_{0}\right) \\ A_{\perp}^{0}: E_{\perp}^{s} \rightleftharpoons \operatorname{range}\left(I d-\pi_{0}\right)\end{cases}
$$

We can choose in view of (2.16), for $|\tau|$ small enough

$$
\begin{cases}A_{\perp}^{0} & =\left(I d-\pi_{0}\right) j_{\perp} \\ A_{0}^{0} & =\pi_{0} j_{0} \alpha\end{cases}
$$

where $\alpha=\alpha\left(x, \tau, \xi^{\prime}\right)$ is the unique endomorphism of $E_{0}$, such that

$$
\oint_{\mathbb{T}^{d}} A_{0}^{0}=\oint_{\mathbb{T}^{d}} \pi_{0} j_{0} \alpha=I d_{E_{0}}
$$

(This choice of $A_{0}^{0}$ will insure the consistency with the definition (0.36) of $p_{0}$.)

The maps $L^{0}\left(x, \tau, \xi^{\prime}\right): E_{0} \rightarrow E_{0}$ and $L_{\perp}^{0}\left(x, \tau, \xi^{\prime}\right): E_{\perp}^{s} \rightarrow E_{\perp}^{s-1}$ are then uniquely determined by (2.12). $L_{\perp}^{0}$ is a smooth function of $\left(x, \tau, \xi^{\prime}\right)$ defined near $\tau=\xi^{\prime}=0$, taking its values in the set of pseudo-differential operators of order 1 for the scale $\left\{E_{\perp}^{s}\right\}$ on the torus: for any $w_{\perp} \in \cup_{s} E_{\perp}^{s}$ one has

$$
\mathbb{M}^{0} j_{\perp}\left(w_{\perp}\right)-j_{\perp} L_{\perp}^{0}\left(w_{\perp}\right)=\mathbb{M}^{0} \pi_{0} j_{\perp}\left(w_{\perp}\right)-\pi_{0} j_{\perp} L_{\perp}^{0}\left(w_{\perp}\right) \in \bigcap_{s} E^{s} .
$$

The map $A^{0}=A_{0}^{0} \oplus A_{\perp}^{0}$

$$
E^{s}=E_{0} \oplus E_{\perp}^{s} \stackrel{A^{0}}{\longrightarrow} E^{s}
$$

is an isomorphism; by (2.16) it satisfies

$$
\left\|A^{0}-I d\right\|_{E^{s}} \leq \text { Cte } \tau^{2} \quad(\forall s \in I) .
$$


The equation (2.13) is equivalent to

$$
\begin{cases}\mathbb{M}^{0} A_{0}^{k}-A_{0}^{k} L^{0}-A_{0}^{0} L^{k}=R_{0}^{k}, & R_{0}^{k} \text { bounded from } E_{0} \text { to } E^{s} \\ \mathbb{M}^{0} A_{\perp}^{k}-A_{\perp}^{k} L_{\perp}^{0}-A_{\perp}^{0} L_{\perp}^{k}=R_{\perp}^{k}, & R_{\perp}^{k} \text { bounded from } E_{\perp}^{s} \text { to } E^{s-1}\end{cases}
$$

where the right hand side is given by induction by the formula

$$
k \geq 1 \quad R_{0, \perp}^{k}=\sum_{\substack{j+\ell+|\alpha|=k \\ j \neq k, \ell \neq k}} \frac{1}{\alpha !} \partial_{\xi^{\prime}}^{\alpha} A_{0, \perp}^{j} \partial_{x^{\prime}}^{\alpha} L_{\cdot, \perp}^{\ell}-\sum_{\substack{j+\ell+|\alpha|=k \\ \ell \neq k}} \frac{1}{\alpha !} \partial_{\xi^{\prime}}^{\alpha} \mathbb{M}^{j} \partial_{x^{\prime}}^{\alpha} A_{0, \perp}^{\ell}-i \partial_{x_{d}} A_{0, \perp}^{k-1} .
$$

Let $A^{k}=A_{0}^{k} \oplus A_{\perp}^{k}, \widetilde{A}^{k}=\left(A^{0}\right)^{-1} A^{k}, \mathcal{L}^{k}=L^{k} \oplus L_{\perp}^{k}, R^{k}=R_{0}^{k} \oplus R_{\perp}^{k}$ and $\widetilde{R}^{k}=\left(A^{0}\right)^{-1} R_{k}$. The equation (2.23) can be rewritten $\mathbb{M}^{0} A^{k}-A^{k} \mathcal{L}^{0}-A^{0} \mathcal{L}^{k}=R^{k}$, which is equivalent by $(2.12)\left[\mathbb{M}^{0} A^{0}=A^{0} \mathcal{L}^{0}\right]$ to $\mathcal{L}^{0} \widetilde{A}^{k}-\widetilde{A}^{k} \mathcal{L}_{0}-\mathcal{L}^{k}=\widetilde{R}^{k}$. The matrix form of this equation on $E_{0} \oplus E_{\perp}^{s}$ is

$$
\begin{gathered}
\left\{\begin{array}{l}
L^{0}\left(\widetilde{A}^{k}\right)_{1,1}-\left(\widetilde{A}^{k}\right)_{1,1} L^{0}=L^{k}+\left(\widetilde{R}^{k}\right)_{1,1} \\
L_{\perp}^{0}\left(\widetilde{A}^{k}\right)_{2,2}-\left(\widetilde{A}^{k}\right)_{2,2} L_{\perp}^{0}=L_{\perp}^{k}+\left(\widetilde{R}^{k}\right)_{2,2}
\end{array}\right. \\
\left\{\begin{array}{l}
L^{0}\left(\widetilde{A}^{k}\right)_{1,2}-\left(\widetilde{A}^{k}\right)_{1,2} L_{\perp}^{0}=\left(\widetilde{R}^{k}\right)_{1,2} \\
L_{\perp}^{0}\left(\widetilde{A}^{k}\right)_{2,1}-\left(\widetilde{A}^{k}\right)_{2,1} L^{0}=\left(\widetilde{R}^{k}\right)_{2,1} .
\end{array}\right.
\end{gathered}
$$

The choice $\left(\widetilde{A}^{k}\right)_{1,1}=0,\left(\widetilde{A}^{k}\right)_{2,2}=0$ gives then $L^{k}, L_{\perp}^{k}$ by $(2.25)$. The unique solvability of $(2.26)$ is a consequence of (0.34) which implies for $|\tau|+\left|\xi^{\prime}\right|$ small enough

$$
\left\{\begin{array}{l}
L_{\perp}^{0} \text { is invertible and }\left\|\left(L_{\perp}^{0}\right)^{-1} ; E_{\perp}^{s-1} \rightarrow E_{\perp}^{s}\right\| \leq C \quad(\forall s \in I) \\
\text { Spectrum }\left(L^{0}\right) \subset\left\{z \in \mathbb{C} ;|z| \leq \operatorname{Cte}\left(|\tau|+\left|\xi^{\prime}\right|\right)\right\}
\end{array}\right.
$$

Thus, solving the second equation in (2.26) is equivalent to find a linear map $u: E_{0}=\mathbb{C}^{2} \rightarrow \bigcap_{s \in I} E_{\perp}^{s}=E_{\perp}^{\sigma_{0}}$ such that

$$
u-\left(L_{\perp}^{0}\right)^{-1} \circ u \circ L^{0}=v
$$

where $v: E_{0} \rightarrow E_{\perp}^{\sigma_{0}}$ is given and (2.27) implies for $|\tau|+\left|\xi^{\prime}\right|$ small the existence of a unique solution $u$ to (2.28). The first equation in (2.26) can be reduced to the second one by taking adjoints.

Remark. We have chosen to work with a fixed interval of regularity on the torus, $s \in\left[-\sigma_{0}, \sigma_{0}\right]=I$ in order to work in the classical theory of semi-classical (in $\varepsilon$ ) peudo-differential operators with values in bounded operators between Hilbert spaces. On the other hand, the neighborhood of the zero section $\tau=\xi^{\prime}=0$ where the above construction applies may depends on $I$.

In view of (2.22), the tangential pseudo-differential operator $A=A_{0} \oplus A_{\perp}$ is elliptic near the zero section $\tau=0, \xi^{\prime}=0$. Let $A^{-1}$ be a pseudo-differential inverse and $\mathcal{L}=L \oplus L_{\perp}$.

By construction we have $\left(\varepsilon \partial_{x_{d}}+\mathbb{M}\right) A \equiv A\left(\varepsilon \partial_{x_{d}}+\mathcal{L}\right)$ near the zero section, and $\mathcal{L}$ is diagonal in the decomposition $E_{0} \oplus E_{\perp}$. Therefore, one deduces that the following identity holds true near the zero section

$$
\oint_{\mathbb{T}^{d}} A^{-1}\left(\varepsilon \frac{\partial}{\partial_{x_{d}}}+\mathbb{M}\right) \equiv\left(\varepsilon \frac{\partial}{\partial_{x_{d}}}+L\right) \oint_{\mathbb{T}^{d}} A^{-1}
$$


If we choose $W_{1} \Subset W_{0}$ two sufficiently small neighborhoods of the set $\left\{\xi^{\prime}=0, \tau=0\right\} \times\left\{t \in\left[-T_{0}, 2 T_{0}\right]\right\}$ in $T^{*} X$, and $Q_{0}, Q_{1}$ two scalars tangentials o.p.d., with $S E\left(Q_{j}\right) \subset W_{j}, j=0,1$, such that $Q_{0} \equiv I d$ on $\bar{W}_{1}$, we then get, with

$$
\begin{gathered}
\Pi_{0} \stackrel{\text { def }}{=} Q_{0} \oint_{\mathbb{T}^{d}} A^{-1} . \\
\Pi_{0}\left(\varepsilon \frac{\partial}{\partial_{x_{d}}}+\mathbb{M}\right)=\left(\varepsilon \frac{\partial}{\partial_{x_{d}}}+L\right) \Pi_{0}+R
\end{gathered}
$$

where $R$ is such that $\| Q_{1} R ; L^{2}\left(X, E^{s}\right) \rightarrow L^{2}\left(X, \mathbb{C}^{2} \| \in O\left(\varepsilon^{\infty}\right)\right.$ for any $s \in I$.

The principal symbol $p_{0}$ of $\Pi_{0}$ is easy to compute:

If $w=\left(I d-\pi_{0}\right) j_{\perp}\left(w_{\perp}\right)+\pi_{0} j_{0} \alpha\left(w_{(0)}\right)$, one has $\pi_{0}(w)=\pi_{0} j_{0} \alpha\left(w_{(0)}\right)$ and $\left(A^{0}\right)^{-1}(w)=w_{(0)} \oplus w_{\perp}$, so we get $\oint_{\mathbb{T}^{d}}\left(A^{0}\right)^{-1}(w)=w_{(0)}=(\operatorname{using}(2.19)) \oint_{\mathbb{T}^{d}} \pi_{0} j_{0} \alpha\left(w_{(0)}\right)=\oint_{\mathbb{T}^{d}} \pi_{0}(w)$ and we recover the definition formula $(0.36)$ of $p_{0}$, if one takes $\chi_{0}$ equal to the principal symbol of $Q_{0}$.

Lemma 2.1. The tangential o.p.d. $L \simeq \sum_{k}\left(\frac{\varepsilon}{i}\right)^{k} L^{k}\left(x, \tau, \xi^{\prime}\right)$ satisfies

$$
\begin{aligned}
& \text { i) } \quad L \equiv \oint_{\pi^{d}}\left(\mathbb{M}_{\mid \tau=0}\right) j_{0} \quad \text { modulo } \tau^{2} \\
& \text { ii) } \quad L^{0}=\left[\begin{array}{cc}
a_{0}^{-1}(x) a_{1}\left(x, i \xi^{\prime}\right) & -a_{0}^{-1}(x) \\
a_{2}\left(x, i \xi^{\prime}\right)+\underline{\hat{\rho}}(x) \tau^{2} & 0
\end{array}\right]+O\left(\tau^{4}\right) \text {. }
\end{aligned}
$$

Proof. For i), we observe that $\tau^{2}$ is a smooth parameter in the above construction, and that by formulas $(0.27,0.28)$, the restriction $\mathbb{M}_{\mid \tau=0}$ is a constant coefficient operator on the torus $\mathbb{T}_{y}^{d}$.

We thus get $\pi_{0 \mid \tau=0}=\oint_{\mathbb{T}^{d}}, \alpha_{\mid \tau=0}=I d, A_{0 \mid \tau=0}=j_{0}, A_{\perp \mid \tau=0}=j_{\perp}, L_{\mid \tau=0}=\oint_{\mathbb{T}^{d}}\left(\mathbb{M}_{\mid \tau=0}\right) j_{0}, L_{\perp \mid \tau=0}$ $=\left(I d-\oint_{\mathbb{T}^{d}}\right)_{\mid \tau=0}\left(\mathbb{M}_{\mid \tau=0}\right) j_{\perp}$.

One has $\oint_{T^{d}} A_{0}^{0}=I d_{E_{0}}$ and $A_{0}^{0}=j_{0}+O\left(\tau^{2}\right)$, so there exists a map $\theta\left(x, \tau^{2}, \xi^{\prime}\right): E_{0} \rightarrow E_{\perp}$ such that $A_{0}^{0}=j+\tau^{2} \theta$. Using (2.12), we get

$$
L^{0}=\oint_{\mathbb{T}^{d}} \mathbb{M}^{0} A_{0}^{0}
$$

so for any $w \in E_{0}$

$$
L^{0}(w)=\oint_{\mathbb{T}^{d}} \mathbb{M}^{0} j_{0}(w)+\tau^{2} \oint_{\mathbb{T}^{d}} \mathbb{M}^{0} \theta(w) .
$$

The definition formula (0.29) of $\mathbb{M}^{0}$ and (2.34) gives the second part of the lemma.

For $u^{\varepsilon} \in I_{h}^{\varepsilon}$, we define $\underline{u}^{\varepsilon}$ by

$$
\underline{u}^{\varepsilon}=\left[\begin{array}{l}
u_{0}^{\varepsilon} \\
u_{1}^{\varepsilon}
\end{array}\right]=\left[\begin{array}{l}
u^{\varepsilon} \\
A_{0}(x) \varepsilon \partial_{x_{d}} u^{\varepsilon}+\varepsilon A_{1}\left(x, \partial_{x^{\prime}}\right) u^{\varepsilon}
\end{array}\right]
$$

and $w^{\varepsilon}=\mathcal{T}\left(u^{\varepsilon}\right)=T\left(\underline{u}^{\varepsilon}\right)$ by

$$
w^{\varepsilon}=\left[\begin{array}{c}
w_{0}^{\varepsilon} \\
w_{1}^{\varepsilon}
\end{array}\right]=\left[\begin{array}{l}
T\left(u_{0}^{\varepsilon}\right) \\
T\left(u_{1}^{\varepsilon}\right)
\end{array}\right]
$$


where $T$ is the transformation $(0.19)$ :

$$
T(f)(t, x, y)=\sum_{\ell \in \mathbb{Z}^{d}} e^{i \ell(y-\Theta((x) / \varepsilon)} f(t, x) .
$$

Then $w^{\varepsilon}$ satisfies, for $s_{0}<-d / 2$.

$$
\begin{gathered}
w^{\varepsilon}(t, x, y) \in L^{2}\left(\left[t_{1}, t_{2}\right] \times \partial \Omega \times\left[0, r_{0}\right] ;\left[H^{s_{0}}\left(\mathbb{T}^{d}\right)\right]^{2}\right) \quad \forall t_{1}, t_{2} \in \mathbb{R} \\
\left\{\begin{array}{l}
\left.\left(\varepsilon \frac{\partial}{\partial x_{d}}+\mathbb{M}\right) w^{\varepsilon}=0 \quad \text { on } \quad \mathbb{R}_{t} \times \partial \Omega \times\right] 0, r_{0}\left[\times \mathbb{T}_{y}^{d}\right. \\
w_{0 \mid x_{d=0}}^{\varepsilon}=0 .
\end{array}\right.
\end{gathered}
$$

We recall that we define the Bloch wave $\Gamma\left(u^{\varepsilon}\right) \in L^{2}\left(X ; \mathbb{C}^{2}\right)$ by

$$
\Gamma\left(u^{\varepsilon}\right)=\left[\begin{array}{l}
\Gamma_{0}\left(u^{\varepsilon}\right) \\
\Gamma_{1}\left(u^{\varepsilon}\right)
\end{array}\right]=\Pi_{0} \circ T\left(\underline{u}^{\varepsilon}\right) .
$$

Proof of Proposition 1.

(We denote by $C$ various constants which are independent of $\varepsilon, h$.)

For $u^{\varepsilon}=\sum_{0.9 \leq \omega_{n}^{\varepsilon} h \leq 2.1}\left(u_{+, n} e^{i t \omega_{n}^{\varepsilon}}+u_{-, n} e^{i t \omega_{n}^{\varepsilon}}\right) e_{n}^{\varepsilon}(x)$ we put $\left\|u^{\varepsilon}\right\|^{2}=\sum\left|u_{+, n}\right|^{2}+\left|u_{-, n}\right|^{2}$. For any $t_{1}<t_{2}$, there exists a constant $C$ such that for any $\varepsilon, h$ and $u^{\varepsilon} \in I_{h}^{\varepsilon}$ one has

$$
\int_{\Omega} \int_{t_{1}}^{t_{2}}\left|h \nabla u^{\varepsilon}\right|^{2}+\left|h \partial_{t} u^{\varepsilon}\right|^{2} d t d_{g} x \leq C\left\|u^{\varepsilon}\right\|^{2}
$$

Let $\gamma=\varepsilon / h$; we rewrite $(2.41)$ on the form

$$
\int_{\Omega} \int_{t_{1}}^{t_{2}}\left|\varepsilon \nabla u^{\varepsilon}\right|^{2}+\left|\varepsilon \partial_{t} u^{\varepsilon}\right|^{2} d t d_{g} x \leq C \gamma^{2}\left\|u^{\varepsilon}\right\|^{2} .
$$

Let $K=\partial \Omega \times\left[0, T_{0}\right] \times\left[0, r_{0} / 2\right] \times\left\{\xi^{\prime}=0, \tau=0\right\}$ and $Q\left(\varepsilon, t, x, \varepsilon \partial_{x^{\prime}}, \varepsilon \partial_{t}\right)$ be a scalar tangential o.p.d. on $X$, equal to $I d$ near $K$.

Let $\alpha$ small such that the geometric control hypothesis (0.9) holds true for $T_{0}-4 \alpha$, and let $Y=\partial \Omega \times\left[\alpha, T_{0}\right.$ $-\alpha] \times\left[0, r_{0} / 2\right]$. By (2.42), for $\gamma$ small,the $L^{2}$ norm of $u^{\varepsilon}$ on $Y$ is concentrated near the set $\xi^{\prime}=0, \tau=0$ where $Q$ is equal to $I d$; so we get

$$
\left\|u^{\varepsilon}\right\|_{L^{2}(Y)}^{2} \leq C\left[\left\|Q\left(u^{\varepsilon}\right)\right\|_{L^{2}\left(X_{T_{0}}\right)}^{2}+(\gamma+\varepsilon)^{2}\left\|u^{\varepsilon}\right\|^{2}\right] .
$$

By construction of $\Pi_{0}$, one has

$$
\Pi_{0}=Q_{0}\left[\oint_{\mathbb{T}^{d}} I d+R_{0}\left(\varepsilon \partial_{t}\right)+\varepsilon R_{1}\right]
$$

where $R_{0,1}$ are tangential o.p.d. from $L^{2}\left(X ; E^{s}\right)$ in $L^{2}\left(X ; E_{0}\right)(s \in I)$. Therefore we get

$$
\left\|\Gamma\left(u^{\varepsilon}\right)-Q_{0}\left(\begin{array}{c}
u_{0}^{\varepsilon} \\
u_{1}^{\varepsilon}
\end{array}\right)\right\|_{L^{2}\left(X ; E_{0}\right)} \leq C[\gamma+\varepsilon]\left\|u^{\varepsilon}\right\|
$$


(here we have used the fact that $\varepsilon \partial_{t}$ commutes with $T$ and is bounded by $O(\gamma=\varepsilon / h)$ on $\left.I_{h}^{\varepsilon}\right)$. Since $Q Q_{0}$ is equal to Id near $K$, we deduce from $(2.43,2.45)$, for $\gamma_{0}, \varepsilon_{0}$ small enough

$$
\left\|u^{\varepsilon}\right\|_{L^{2}(Y)}^{2} \leq C\left[\left\|Q \Gamma_{0}\left(u^{\varepsilon}\right)\right\|_{L^{2}\left(X_{T_{0}}\right)}^{2}+(\gamma+\varepsilon)^{2}\left\|u^{\varepsilon}\right\|^{2}\right] .
$$

We are now ready to prove $(0.45)$ by a contradiction argument. If (0.45) is untrue, there exist sequences $\varepsilon_{k} \rightarrow 0, \gamma_{k} \rightarrow 0, h_{k} \rightarrow 0, h_{k} \geq \varepsilon_{k} / \gamma_{k}, u^{k} \in I_{h_{k}}^{\varepsilon_{k}}$ such that

$$
\left\{\begin{array}{l}
\left\|u^{k}\right\|=1 \\
\lim _{k \rightarrow \infty}\left\|Q \Gamma_{0}\left(u^{k}\right)\right\|_{L^{2}\left(X_{T_{0}}\right)}^{2}+\left\|u^{k}\right\|_{L^{2}\left(\left(0, T_{0}\right) \times V\right)}^{2}=0 .
\end{array}\right.
$$

Moreover, we can suppose that the weak limit $u=$ weak $-\lim \left(u^{k}\right)$ exist. Then $u$ satisfies $(0.6)$ and is equal to 0 on $\left(0, T_{0}\right) \times V$. By the geometric control hypothesis (0.9) of [4], the estimate (0.7) holds true for $u$, so we get $u=0$. We deduce from $(2.46)$

$$
\lim _{k \rightarrow \infty}\left\|u^{k}\right\|_{L^{2}(Y)}=0
$$

We are thus reduced to an interior problem in $\Omega$.

Let $Z=\left\{x \in \Omega ; \operatorname{dist}(x, \partial \Omega)>r_{0} / 4\right\} \times \mathbb{R}_{t}$. We denote by $\widetilde{\mathbb{M}}=\rho(x, y)\left(\varepsilon \partial_{t}\right)^{2}-\varepsilon^{2}\left(\Delta_{g}\right)_{\varepsilon}^{*}$ the Bloch operator on $Z$, and $G^{s}=H^{s}\left(\mathbb{T}^{d}\right)$. By the same construction as above, there exist a $\varepsilon$-pseudo-differential operator $\widetilde{\Pi}_{0}\left(x, \xi, \tau, y, \partial_{y}\right): L^{2}\left(Z, G^{\bullet}\right) \rightarrow L^{2}(Z, \mathbb{C})$ and a scalar $\varepsilon$-o.p.d. $\widetilde{L}(x, \xi, \tau): L^{2}(Z ; \mathbb{C}) \rightarrow L^{2}(Z, \mathbb{C})$, defined near the zero section $\xi=\tau=0$, such that

$$
\widetilde{\Pi}_{0} \widetilde{\mathbb{M}}=\widetilde{L} \widetilde{\Pi}_{0}+\widetilde{R}
$$

The principal symbol of $\widetilde{\Pi}_{0}$ is $\tilde{\chi}_{0} \oint_{\pi^{d}} \frac{1}{2 i \pi} \int_{\partial D} \frac{d z}{z-\mathbb{M}^{0}}$ with $\tilde{\chi}_{0} \in C_{0}^{\infty}\left(\widetilde{W}_{0}\right), \tilde{\chi}_{0} \equiv 1$ on $\widetilde{W}_{1}$, where $\widetilde{W}_{1} \Subset \widetilde{W}_{0}$ are two small neighborhood of the set $\{\xi=\tau=0\} \times\left\{t \in\left[-T_{0}, 2 T_{0}\right]\right\}$ in $T^{*} Z$. The scalar operator $\widetilde{L}$ satisfies

$$
\left\{\begin{array}{l}
\widetilde{L} \simeq \sum_{k}\left(\frac{\varepsilon}{i}\right)^{k} \widetilde{L}^{k}(x, \tau, \xi) \\
\widetilde{L}_{\mid \tau=0}=-\varepsilon^{2} \Delta_{g} \quad \text { modulo } \tau^{2} \\
\widetilde{L}^{0}(x, \tau, \xi)=-\underline{\rho}(x) \tau^{2}+\|\xi\|^{2}+0\left(\tau^{4}\right) .
\end{array}\right.
$$

The error terms $\widetilde{R}$ in $(2.49)$ is such that for any $\varepsilon$-o.p.d. $\widetilde{Q}$ with essential support in $\widetilde{W}_{1}$, one has

$$
\left\|\widetilde{Q} \circ \widetilde{R} ; L^{2}\left(Z ; G^{s}\right) \rightarrow L^{2}(Z ; \mathbb{C})\right\| \in \mathcal{O}\left(\varepsilon^{\infty}\right) \quad \forall s \in\left[-\sigma_{0}, \sigma_{0}\right] .
$$

Let $v^{k}(t, x, y)$ be the distribution on $Z \times \mathbb{T}^{d}$

$$
v^{k}=T\left(u^{k}\right)=\sum_{\ell \in \mathbb{Z}^{d}} e^{i \ell\left(y-x / \varepsilon_{k}\right)} u^{k}(t, x)
$$

We deduce from (2.50) that $\left(\frac{h}{\varepsilon}\right)^{2} \widetilde{L} \stackrel{\text { def }}{=} \widetilde{\mathcal{L}}$ is an $h$-o.p.d.; writing $\frac{\varepsilon}{i} \partial_{x}=\frac{\varepsilon}{h}\left(\frac{h}{i} \partial_{x}\right)$, and using $\frac{h_{k}}{\varepsilon_{k}} \geq \frac{1}{\gamma_{k}} \rightarrow \infty(2.49$, $2.51)$ we get, for any $h$-o.p.d. $Q$ compactly supported in $\{\xi, \tau\}$ and with support in $Z \times\left\{t \in\left(-T_{0}, 2 T_{0}\right)\right\}$

$$
\left\|Q \widetilde{\mathcal{L}} \widetilde{\Pi}_{0} v^{k}\right\|_{L^{2}(Z)} \in 0\left(h_{k}^{\infty}\right)
$$


By the analogue of (2.45) in the interior case, we also have

$$
\left\|\widetilde{\Pi}_{0} v^{k}-\widetilde{Q}_{0} u^{k}\right\|_{L^{2}\left(Z \cap\left\{t \in\left[-T_{0}, 2 T_{0}\right]\right\}\right)} \leq C\left[\gamma_{k}+\varepsilon_{k}\right]
$$

where $\widetilde{Q}_{0}$ is an $\varepsilon$-o.p.d. with principal symbol $\chi_{0}$, with essential support in $\widetilde{W}_{0}$.

Let $\mu$ be a $h$-semi classical measure associated to $\left\{u^{k}\right\}$ (see [8]). (The hypothesis $u^{k} \in I_{h_{k}}^{\varepsilon_{k}}$ implies that $\mu$ is supported in $|\tau| \in[0.9,2.1]$.) From (2.47) and (2.46) we deduce that

$$
\mu_{\mid Y \cap Z} \equiv 0 \text { and } \mu_{\mid] 0, T_{0}[\times V} \equiv 0 .
$$

Let $\nu$ be a h-semiclassical measure associated to $\widetilde{\Pi}_{0} v^{k}$. Using $(2.54)$ and $\lim _{k \rightarrow \infty} \varepsilon_{k} / h_{k}=0$ we get

$$
\nu=\widetilde{\chi}_{0}^{2}\left(t, x ; \xi^{\prime}=0, \tau=0\right) \mu .
$$

The principal symbol of $\widetilde{\mathcal{L}}$ is $-\underline{\rho}(x) \tau^{2}+\|\xi\|^{2}+\gamma_{k}^{2} 0\left(\tau^{4}\right)$. In the equation (2.53) we view $\gamma_{k}=\varepsilon_{k} / h_{k}$ as a small parameter. We can then use the proof of the interior propagation theorem (see [8]) with the additional parameter $\gamma_{k}$ going to zero. We get from (2.53) that the support of $\nu$ is contained in the set $\underline{\rho}(x) \tau^{2}-\|\xi\|^{2}=0$, and that the support of $\nu$ propagates along the bicharacteristic flow of $\underline{\rho}(x) \tau^{2}-\|\xi\|^{2}$. Using $(2.55,2.56)$, and the hypothesis (0.9) we obtain for $\beta$ small

$$
\mu_{] T_{0} / 2-\beta, T_{0} / 2+\beta[} \equiv 0
$$

Using (2.41), we get that the sequence $u^{k}$ is h-oscillatory (see [7]), so from (2.57) we deduce

$$
\lim _{k \rightarrow \infty}\left\|u^{k}\right\|_{L^{2}(Z \times] T_{0} / 2-\beta, T_{0} / 2+\beta[)} \equiv 0 .
$$

Then from (2.48), we obtain $\lim _{k \rightarrow \infty}\left\|u^{k}\right\|_{L^{2}\left(\Omega \times\left(T_{0} / 2-\beta, T_{0} / 2+\beta\right)\right)}=0$ which contredicts $\left\|u^{k}\right\| \equiv 1$.

\section{Lopatinski estimate}

\subsection{Proof of Proposition 2}

We first verify the implication Theorem $2 \Rightarrow$ Proposition 2. For $u^{\varepsilon} \in I_{h}^{\varepsilon}$, we have

$$
w^{\varepsilon}=\left[\begin{array}{l}
w_{0}^{\varepsilon} \\
w_{1}^{\varepsilon}
\end{array}\right]=\left[\begin{array}{l}
T\left(u^{\varepsilon}\right) \\
T\left(A_{0}\left(\varepsilon \partial_{x_{d}} u^{\varepsilon}\right)+\varepsilon A_{1}\left(x, \partial_{x^{\prime}}\right) u^{\varepsilon}\right)
\end{array}\right]
$$

and by $(2.44)$

$$
\Gamma\left(u^{\varepsilon}\right)=Q_{0}\left[\oint_{\mathbb{T}^{d}} w^{\varepsilon}+R_{0}\left(\varepsilon \partial_{t}\right) w^{\varepsilon}+\varepsilon R_{1} w^{\varepsilon}\right]
$$

The Dirichlet boundary condition $\left.u^{\varepsilon}\right|_{x_{d}=0}$ implies $\left.w_{0}^{\varepsilon}\right|_{x_{d}=0}=0$, so we get

$$
\left.\Gamma_{0}\left(u^{\varepsilon}\right)\right|_{x_{d}=0}=Q_{0}\left[\oint_{\mathbb{T}^{d}}\left(R_{0}\left(\varepsilon \partial_{t}\right)+\varepsilon R_{1}\right)\left[\begin{array}{l}
0 \\
\left.w_{1}^{\varepsilon}\right|_{x_{d}=0}
\end{array}\right]\right]_{1^{s t} \text { component }} .
$$


If one multiplies the equation (0.4) by $\varepsilon^{3} \varphi\left(x_{d}\right) \frac{\partial}{\partial x_{d}}$ where $\varphi \in C_{0}^{\infty}(]-r_{0} / 2, r_{0} / 2[)$ is equal to 1 near the boundary $x_{d}=0$, and integrates by part, one gets

$$
\begin{cases}\text { For any } t_{1}, t_{2}, \text { there exist } \quad C \text { s.t. } & \forall \varepsilon \\ \left\|\varepsilon \partial_{n} u^{\varepsilon}\right\|_{L^{2}\left(\left(t_{1}, t_{2}\right) \times \partial \Omega\right)} \leq C \varepsilon^{-1 / 2}\left\|u^{\varepsilon}\right\| & \forall u^{\varepsilon} \in I_{h}^{\varepsilon} .\end{cases}
$$

Therefore, by (3.1) we get for $s_{0}<-d / 2$

$$
\|\left. w_{1}^{\varepsilon}\right|_{x_{d=0}} ; L^{2}\left(\left(t_{1}, t_{2}\right) \times \partial \Omega ; H^{s_{0}}\left(\mathbb{T}_{y}^{d}\right)\left\|\leq C \varepsilon^{-1 / 2}\right\| u^{\varepsilon} \| .\right.
$$

If $R$ is an o.p.d from $L^{2}\left(X_{T_{0} \cap x_{d=0}} ; H^{s_{0}}\left(\mathbb{T}^{d}\right)\right)$ in $L^{2}\left(X_{T_{0} \cap x_{d=0}}\right)$, using the classical calculus of Appendix A.1, we get from the a priori bound (3.5) on the trace $\left.w_{1}^{\varepsilon}\right|_{x_{d=0}}$

$$
\left\|\left.\left[Q_{0}, R\right] w_{1}^{\varepsilon}\right|_{x_{d=0}} ; L^{2}\left(X_{T_{0} \cap x_{d=0}}\right)\right\| \leq C \varepsilon^{1 / 2}\left\|u^{\varepsilon}\right\| .
$$

If Theorem 2 holds true, we have

$$
\left\|\left.Q_{0} w_{1}^{\varepsilon}\right|_{x_{d=0}} ; L^{2}\left(X_{T_{0} \cap x_{d=0}} ; H^{s_{1}}\left(\mathbb{T}^{d}\right)\right)\right\| \leq C\left\|u^{\varepsilon}\right\| .
$$

Now using the fact that $\varepsilon \partial_{t}$ commutes with $T$ and is bounded by $\mathcal{O}(\gamma=\varepsilon / h)$ on $I_{h}^{\varepsilon},(3.3,3.6,3.7)$ and $\varepsilon \leq h_{0} \varepsilon / h$, we get $(0.47)$, i.e.

$$
\left\|\left.\Gamma_{0}\left(u^{\varepsilon}\right)\right|_{x_{d=0}} ; L^{2}\left(X_{T_{0}} \cap x_{d}=0\right)\right\| \leq C \frac{\varepsilon}{h}\left\|u^{\varepsilon}\right\| .
$$

\subsection{Proof of Theorem 2}

In this part, we work with a family $\left\{u^{\varepsilon}\right\}_{\varepsilon}, u^{\varepsilon} \in I_{h}^{\varepsilon}$ with $\left.\left.\varepsilon \in\right] 0, \varepsilon_{0}\right], h \in\left[\varepsilon / \gamma_{0}, h_{0}\right]$; we always assume $\left\|u^{\varepsilon}\right\| \leq 1$. We first remark that the Theorem 2 is local near any $\rho_{0}=\left(t_{0}, x_{0}^{\prime}, \tau_{0}=0, \xi_{0}^{\prime}=0\right) \in T^{*}\left(\mathbb{R}_{t} \times \partial \Omega\right)$. Let $Q_{1}$ be a tangential scalar o.p.d equal to $I d$ near $\rho_{0}$, and with essential support close to $\rho_{0}$, and contained in $W_{0}$. By $(2.38,2.39)$ we get (see $(0.30)$ for the definition of $\left.E^{s}\right)$

$$
\left\{\begin{array}{l}
\left(\varepsilon \frac{\partial}{\partial x_{d}}+\mathbb{M}^{0}\right) Q_{1} w^{\varepsilon}=\tilde{g}^{\varepsilon} \\
\tilde{g}^{\varepsilon}=\left[\varepsilon \frac{\partial}{\partial x_{d}}+\mathbb{M}, Q_{1}\right] w^{\varepsilon}-\frac{\varepsilon}{i} \sum_{j=1}^{2} \mathbb{M}^{j} Q_{1} w^{\varepsilon}
\end{array}\right.
$$

and for any $s_{0}+1<-d / 2$ and any $t_{1}, t_{2}$

$$
\begin{gathered}
\sup _{\varepsilon} \| Q_{1} w^{\varepsilon} ; L^{2}\left(\left[t_{1}, t_{2}\right] \times \partial \Omega \times\left[0, r_{0}\right] ; E^{s_{0}+1} \|<+\infty\right. \\
\sup _{\varepsilon} \varepsilon^{-1} \| \tilde{g}^{\varepsilon} ; L^{2}\left(\left[t_{1}, t_{2}\right] \times \partial \Omega \times\left[0, r_{0}\right] ; E^{s_{0}} \|<+\infty .\right.
\end{gathered}
$$

We define $f^{\varepsilon}, g^{\varepsilon}$ by

$$
Q_{1} w^{\varepsilon}=\left(\begin{array}{c}
f_{0}^{\varepsilon} \\
i f_{1}^{\varepsilon}
\end{array}\right), f^{\varepsilon}=\left(\begin{array}{c}
f_{0}^{\varepsilon} \\
f_{1}^{\varepsilon}
\end{array}\right), g^{\varepsilon}=\left(\begin{array}{c}
\tilde{g}_{0}^{\varepsilon} \\
i \\
-\tilde{g}_{1}^{\varepsilon}
\end{array}\right) .
$$


We may assume that $f^{\varepsilon}$ is supported in a small neighborhood $U=U_{0} \times\left[0, r_{1}\right.$ [ of $\left(t_{0}, x_{0}^{\prime}\right)$ in $\mathbb{R}_{t} \times \partial \Omega \times\left[0, r_{0}\right]$, and we denote by $\left(x_{1}, \cdots, x_{d-1}\right)$ a local coordinate system near $x_{0}^{\prime}$ in $\partial \Omega$. Near the boundary by the choice of coordinates $(0.15)$, we have $a_{0}(x) \equiv 1$ and $a_{1}\left(x, \xi^{\prime}\right) \equiv 0$, so equation (3.8) may be rewritten as

$$
\left\{\begin{array}{l}
\frac{\varepsilon}{i} \frac{\partial}{\partial x_{d}} f^{\varepsilon}+\mathbb{N} f^{\varepsilon}=g^{\varepsilon} \\
\mathbb{N}=\left(\begin{array}{cc}
e_{d}(x) \cdot D_{y} & -1 \\
a_{2}\left(x, \frac{\varepsilon}{i} \partial_{x^{\prime}}+e^{\prime}(x) D_{y}\right)-\hat{\rho}(x, y)\left(\frac{\varepsilon \partial_{t}}{i}\right)^{2} & e_{d}(x) \cdot D_{y}
\end{array}\right)
\end{array}\right.
$$

with $D_{y}=\frac{1}{i} \frac{\partial}{\partial y}, e^{\prime}(x) D_{y}=\left(e_{1}(x) \cdot D_{y}, \cdots, e_{d-1}(x) . D_{y}\right)$, we define the trace operators $\operatorname{Tr}_{0}, \operatorname{Tr}_{1}$ by

$$
\operatorname{Tr}_{0}\left(f^{\varepsilon}\right)=\left.f_{0}^{\varepsilon}\right|_{x_{d}=0} \quad \operatorname{Tr}_{1}\left(f^{\varepsilon}\right)=\left.f_{1}^{\varepsilon}\right|_{x_{d}=0}
$$

We have $\operatorname{Tr}_{0}\left(f^{\varepsilon}\right) \equiv 0$ and we have to prove

$$
\left\{\begin{array}{l}
\text { If } W_{0} \subset\left\{\left|\xi^{\prime}\right|+|\tau|<\alpha_{0}\right\}, \text { with } \alpha_{0} \text { small enough, there exist } s_{1}, C, \text { s.t. } \\
\sup _{\varepsilon}\left\|\operatorname{Tr}_{1}\left(f^{\varepsilon}\right) ; L^{2}\left(U_{0} ; H^{s_{1}}\left(\mathbb{T}^{d}\right)\right)\right\| \leq C .
\end{array}\right.
$$

For any $\ell \in \mathbb{Z}^{d}$, we define $\ell_{x}^{\perp}$ and $\ell_{x}^{\prime \prime}$

$$
\ell_{x}^{\perp}=e_{d}(x) \cdot \ell, \ell_{x}^{\prime \prime}=\left(e_{1}(x) \cdot \ell, \cdots, e_{d-1}(x) \cdot \ell\right) .
$$

We have by (32), with $\left\|\ell_{x}^{\prime \prime}\right\|^{2}=a_{2}\left(x, \ell_{x}^{\prime \prime}\right)$

$$
\left\|^{t} d \theta(x)(\ell)\right\|_{x}^{2}=\left(\ell_{x}^{\perp}\right)^{2}+\left\|\ell_{x}^{\prime \prime}\right\|^{2} .
$$

Let $\mathbb{N}_{0}(x)$ be the restriction of $\mathbb{N}$ to the zero section $\xi^{\prime}=\tau=0$. We have

$$
\left\{\begin{array}{l}
\mathbb{N}_{0, \ell}(x)=\left(\begin{array}{cc}
\ell_{x}^{\perp} & -1 \\
\left\|\ell_{x}^{\prime \prime}\right\|^{2} & \ell_{x}^{\perp}
\end{array}\right) \in \mathcal{M}_{2}(\mathbb{R}) \\
\mathbb{N}_{0}(x)\left(\sum_{\ell} z_{\ell} e^{i \ell y}\right)=\sum_{\ell} \mathbb{N}_{0, \ell}(x)\left(z_{\ell}\right) e^{i \ell y}
\end{array}\right.
$$

and the eigenvalues of $\mathbb{N}_{0, \ell}(x)$ are

$$
\lambda_{ \pm, \ell}^{0}(x)=\ell_{x}^{\perp} \pm i\left\|\ell_{x}^{\prime \prime}\right\|
$$

Our strategy of proof of the estimate (3.14) is to split $f^{\varepsilon}$ into two pieces. The first one will be concentrate near $\left\|\ell_{x}^{\prime \prime}\right\|$ small, where the spectrum of $\mathbb{N}$ is close to the real axis; we shall treat this part by a perturbation argument on the spectral theory of $\mathbb{N}$. The second one $\left\|\ell_{x}^{\prime \prime}\right\| \geq c^{t e}>0$ will be handle by elliptic estimates on $\mathbb{N}$.

To achieve this program, we shall use the "exotic" pseudo-differential calculus of Appendice A.2, with $Z=\mathbb{R}_{t} \times \mathbb{R}_{x^{\prime}}^{d-1} \times\left[0, r_{0}\right]_{x_{d}}$; to simplify notation we denote by $\mathcal{S}^{t, m}$ (resp. $\mathcal{B}^{t, m}$ ) the class of symbols (resp. operators) defined in (A.15) (resp. (A.17)). The restriction on $x_{d}=0$ of these class of symbols and operators will be denoted by $\mathcal{S}^{m}, \mathcal{B}^{m}$. 
We first conjugate the equation (3.12) so that the natural scale of space on the torus will be

$$
\mathcal{H}^{s} \stackrel{\text { def }}{=}\left[H^{s}\left(\mathbb{T}^{d}\right)\right]^{2}
$$

Let $\left\langle\ell_{x}^{\prime \prime}\right\rangle=\left(1+\left\|\ell_{x}^{\prime \prime}\right\|^{2}\right)^{1 / 2}$. We define the operators $\Lambda=\Lambda(x)$ and $\mathbb{E}_{0}=\mathbb{E}_{0}(x)$ on the torus by

$$
\begin{gathered}
\Lambda\left(\sum_{\ell} z_{\ell} e^{i \ell y}\right)=\sum_{\ell}\left(\begin{array}{cc}
1 & 0 \\
0 & \left\langle\ell_{x}^{\prime \prime}\right\rangle
\end{array}\right)\left(z_{\ell}\right) e^{i \ell y} \\
\mathbb{E}_{0}\left(\sum_{\ell} z_{\ell} e^{i \ell y}\right)=\sum_{\ell}\left(\begin{array}{cc}
\ell_{x}^{\perp} & -\left\langle\ell_{x}^{\prime \prime}\right\rangle \\
\frac{\left\|\ell_{x}^{\prime \prime}\right\|^{2}}{\left\langle\ell_{x}^{\prime \prime}\right\rangle} & \ell_{x}^{\perp}
\end{array}\right)\left(z_{\ell}\right) e^{i \ell y} .
\end{gathered}
$$

Let $F^{\varepsilon}$ be

$$
F^{\varepsilon}=\Lambda^{-1}\left(f^{\varepsilon}\right)
$$

We have $\operatorname{Tr}_{0}\left(F^{\varepsilon}\right)=0$ and by (3.9), and the fact that $\Lambda^{-1}$ maps clearly $E^{s+1}$ in $\mathcal{H}^{s}$, we get

$$
\sup _{\varepsilon}\left\|F^{\varepsilon} ; L^{2}\left(U ; \mathcal{H}^{s_{0}}\right)\right\|<\infty .
$$

Lemma 3.1. There exist $q \in \mathcal{S}^{t, 0}$, with

$$
q_{\mid \xi^{\prime}=0, \tau=0} \equiv 0
$$

such that, for any scalar tangential symbol $\theta\left(t, x, \tau, \xi^{\prime}\right)$ equal to Id near the essential support of $Q_{1}$ and with support in $\left\{\left|\xi^{\prime}\right|+|\tau| \leq \alpha_{0}\right\}, F^{\varepsilon}$ satisfies

$$
\begin{gathered}
G^{\varepsilon}=\frac{\varepsilon}{i} \frac{\partial}{\partial x_{d}} F^{\varepsilon}+\left(\mathbb{E}_{0}+\left(\begin{array}{cc}
0 & 0 \\
O p(q \theta) & 0
\end{array}\right)\right) F^{\varepsilon} \\
\sup _{\varepsilon} \varepsilon^{-1}\left\|G^{\varepsilon} ; L^{2}\left(U ; \mathcal{H}^{s_{0}-1}\right)\right\|<+\infty .
\end{gathered}
$$

Proof. We conjugate (3.12) by $\Lambda$ and we obtain

$$
\frac{\varepsilon}{i} \frac{\partial}{\partial x_{d}} F^{\varepsilon}+\Lambda^{-1} \mathbb{N} \Lambda F^{\varepsilon}=\Lambda^{-1} g^{\varepsilon}-\Lambda^{-1} \frac{\varepsilon}{i}\left(\frac{\partial}{\partial x_{d}} \Lambda\right) F^{\varepsilon} .
$$

We have

$$
\left(\frac{\partial}{\partial x_{d}} \Lambda\right)\left(\sum_{\ell} z_{\ell} e^{i \ell y}\right)=\sum_{\ell}\left(\begin{array}{cc}
0 & 0 \\
0 & \frac{\partial}{\partial x_{d}}\left\langle\ell_{x}^{\prime \prime}\right\rangle
\end{array}\right)\left(z_{\ell}\right) e^{i \ell y}
$$

and $\left|\frac{\partial}{\partial x_{d}}\left\langle\ell_{x}^{\prime \prime}\right\rangle\right| \leq c^{t e}\left(1+|\ell|^{2}\right)^{1 / 2}$; therefore (by $(3.10,3.19)$ ) we get

$$
\sup _{\varepsilon} \varepsilon^{-1}\left\|\Lambda^{-1} g^{\varepsilon}-\Lambda^{-1} \frac{\varepsilon}{i}\left(\frac{\partial}{\partial x_{d}} \Lambda\right) F^{\varepsilon} ; L^{2}\left(U ; \mathcal{H}^{s_{0}-1}\right)\right\|<+\infty .
$$


A simple computation gives

$$
\left\{\begin{array}{l}
\Lambda^{-1} \mathbb{N} \Lambda=\mathbb{E}_{0}+\left(\begin{array}{ll}
0 & 0 \\
R & 0
\end{array}\right) \\
R=O p\left(\underset{\ell}{\oplus}\left\langle\ell_{x}^{\prime \prime}\right\rangle\right)^{-1}\left[a_{2}\left(x, \frac{\varepsilon}{i} \frac{\partial}{\partial x^{\prime}}\right)+\sum_{j=1}^{d-1} \frac{\partial a_{2}}{\partial \xi_{j}^{\prime}}\left(x, \frac{\varepsilon}{i} \frac{\partial}{\partial x^{\prime}}\right)\left(e_{j}(x) . D_{y}\right)+\hat{\rho}(x, y)\left(\varepsilon \partial_{t}\right)^{2}\right]
\end{array}\right.
$$

with

$$
O p\left(\underset{\ell}{\oplus}\left\langle\ell_{x}^{\prime \prime}\right\rangle\right)^{-1}\left(\sum_{\ell} z_{\ell} e^{i \ell y}\right)=\sum_{\ell}\left\langle\ell_{x}^{\prime \prime}\right\rangle^{-1} z_{\ell} e^{i \ell y}
$$

Let $\theta\left(t, x, \tau, \xi^{\prime}\right)$ be a classical tangential o.p.d. with support in $\left\{\left|\xi^{\prime}\right|+|\tau| \leq \alpha_{0}\right\}$ and equal to $I d$ near the essential support of $Q_{1}$. By (3.11) we have

$$
\left\|O p(\theta) F^{\varepsilon}-F^{\varepsilon} ; L^{2}\left(U, \mathcal{H}^{s_{0}}\right)\right\| \in \mathcal{O}\left(\varepsilon^{\infty}\right) .
$$

Therefore we can move $R\left(\left(1-O p(\theta) F^{\varepsilon}\right)\right.$ from the left to the right of $(3.27)$. So we just have to verify

$$
R \circ O p(\theta)=O p(q \theta)+\varepsilon O p\left(\underset{\ell}{\oplus}\left\langle\ell_{x}^{\prime \prime}\right\rangle\right)^{-1} \circ O p(b)
$$

with $q \in \mathcal{S}^{t, 0}$ so that (3.24) holds true, and $b \in \mathcal{S}^{t, 1}$. The $b$ term in (3.31) is defined by $\left[O p\left(a_{2}\left(x, \xi^{\prime}\right)+\cdots\right] \circ\right.$ $O p(\theta)=O p\left(\theta\left(a_{2}+\cdots\right)\right)+\varepsilon O p(b)$ and belongs clearly to $\mathcal{S}^{t, 1}$ (there is no loose in the $x$ derivatives of $b$ in (A.15)). Let $\chi\left(\tau, \xi^{\prime}\right) \in C_{0}^{\infty}$ equal to 1 for $\left(|\tau|+\left|\xi^{\prime}\right|\right) \leq 2 \alpha_{0}$. We define $q$ by

$$
\left.q=\underset{\ell}{(}\left\langle\ell_{x}^{\prime \prime}\right\rangle^{-1}\right)\left[a_{2}\left(x, \xi^{\prime}\right)+\sum_{j=1}^{d-1} \frac{\partial a_{2}}{\partial \xi_{j}^{\prime}}\left(x, \xi^{\prime}\right)\left(e_{j}(x) \cdot D_{y}\right)-\hat{\rho}(x, y) \tau^{2}\right] \cdot \chi\left(\tau, \xi^{\prime}\right) .
$$

The estimates $\left|e_{j}(x) \cdot \ell\right| \leq C^{t e}\left\langle\ell_{x}^{\prime \prime}\right\rangle, j \leq d-1$, and

$$
\forall \alpha \exists C_{\alpha}\left|\partial_{x}^{\alpha}\left(\left\langle\ell_{x}^{\prime \prime}\right\rangle^{-1}\right)\right| \leq C_{\alpha}(1+|\ell|)^{|\alpha|}\left(\left\langle\ell_{x}^{\prime \prime}\right\rangle^{-1}\right)
$$

implies $q \in \mathcal{S}^{t, 0}$. The function $a_{2}\left(x, \xi^{\prime}\right)$ is quadratic in $\xi^{\prime}$ so (3.24) follows from (3.32).

The eigenvalues of $\mathbb{E}_{0}=\Lambda^{-1} \mathbb{N}_{0} \Lambda$ are $\lambda_{ \pm, \ell}^{0}(x)=\ell_{x}^{\perp} \pm i\left\|\ell_{x}^{\prime \prime}\right\|$. For any $x$, the set $\left(e_{1}(x), \cdots, e_{d}(x)\right)$ is a basis of $\mathbb{R}^{d}$, so by the definition (3.15) of $\ell_{x}^{\perp}$ and $\ell_{x}^{\prime \prime}$, there exist $c_{1}>0$ such that

$$
\left|\ell_{x}^{\perp}-k_{x}^{\perp}\right|+\left\|\ell_{x}^{\prime \prime}-k_{x}^{\prime \prime}\right\| \geq 4 c_{1}|\ell-k| \quad \forall x, \forall k, \ell \in \mathbb{Z}^{d} .
$$

This implies the following separation property for the spectrum of $\mathbb{E}_{0}$ near the real axis

Lemma 3.2. For any $x, \ell \in \mathbb{Z}^{d}$ such that $\left\|\ell_{x}^{\prime \prime}\right\| \leq c_{1}$, one has

$$
\operatorname{dist}\left(\left\{\lambda_{ \pm, \ell}^{0}(x)\right\},\left\{\lambda_{ \pm, k}^{0}(x)\right\}\right) \geq c_{1} \quad \forall k \neq \ell .
$$

Proof. If (3.35) is false, one has $\left|\ell_{x}^{\perp}-k_{x}^{\perp}\right|<c_{1}$ and

$$
\left|\left\|\ell_{x}^{\prime \prime}\right\|-\left\|k_{x}^{\prime \prime}\right\|\right|<c_{1} \text {, so we get }\left|\ell_{x}^{\perp}-k_{x}^{\perp}\right|+\left\|\ell_{x}^{\prime \prime}-k_{x}^{\prime \prime}\right\|<c_{1}+3 c_{1}
$$

in contradiction with (3.34). 
Let $S p_{0}(x)$ be the spectrum of $\mathbb{E}_{0}(x)$

$$
S p_{0}(x)=\bigcup_{ \pm, \ell} \lambda_{ \pm, \ell}^{0}(x)
$$

By (3.21), for $\lambda \notin S p_{0}(x)$ the resolvant $\left(\lambda-\mathbb{E}_{0}(x)\right)^{-1}$ is diagonal with respect to the decomposition $\underset{\ell}{\oplus} e^{i \ell y} \mathbb{C}^{2}$, $\left(\lambda-\mathbb{E}_{0}(x)\right)^{-1}=\underset{\ell}{\oplus}\left(\lambda-\mathbb{E}_{0, \ell}(x)\right)^{-1}$ with

$$
\left(\lambda-\mathbb{E}_{0, \ell}(x)\right)^{-1}=\frac{1}{\left(\lambda-\lambda_{+, \ell}^{0}\right)\left(\lambda-\lambda_{-, \ell}^{0}\right)}\left(\begin{array}{cc}
\lambda-\ell_{x}^{\perp} & -\left\langle\ell_{x}^{\prime \prime}\right\rangle \\
\frac{\left\|\ell_{\ell}^{\prime \prime}\right\|^{2}}{\left\langle\ell_{x}^{\prime \prime}\right\rangle} & \lambda-\ell_{x}^{\perp}
\end{array}\right) .
$$

Lemma 3.3. For any $c_{0}>0$, there exist $M$ such that for any $x$, $\operatorname{dist}\left(\lambda, S p_{0}(x)\right) \geq c_{0}$ implies

$$
\left\|\left(\lambda-\mathbb{E}_{0, \ell}(x)\right)^{-1}\right\| \leq M \quad \forall \ell .
$$

Proof. We may suppose $\operatorname{Im} \lambda \geq 0$. Then we have $\left|\lambda-\lambda_{+, \ell}^{0}\right|\left|\lambda-\lambda_{-, \ell}^{0}\right| \geq c_{0}\left|\lambda-\ell_{x}^{\perp}+i\left\|\ell_{x}^{\prime \prime}\right\|\right|$, so

$$
\frac{\left|\lambda-\ell_{x}^{\perp}\right|}{\left|\lambda-\lambda_{+, \ell}^{0}\right|\left|\lambda-\lambda_{-, \ell}^{0}\right|} \leq \frac{1}{c_{0}}
$$

and

$$
\frac{c_{0}\left\langle\ell_{x}^{\prime \prime}\right\rangle}{\left|\lambda-\lambda_{+, \ell}^{0} \| \lambda-\lambda_{-, \ell}^{0}\right|} \leq \frac{\sqrt{1+\left\|\ell_{x}^{\prime \prime}\right\|^{2}}}{\max \left(c_{0},\left\|\ell_{x}^{\prime \prime}\right\|\right)} .
$$

The lemma follows from these two inequalities by (3.37).

Let us define $\mathbb{E}=\mathbb{E}\left(t, x, \tau, \xi^{\prime}\right)$ by (see $\left.(3.25)\right)$

$$
\mathbb{E}=\mathbb{E}_{0}+\left(\begin{array}{cc}
0 & 0 \\
q \theta & 0
\end{array}\right)
$$

For $\beta>0$, let $\Sigma_{\beta}(x) \subset \mathbb{Z}^{d}$ be the set

$$
\Sigma_{\beta}(x)=\left\{\ell \in \mathbb{Z}^{d},\left\|\ell_{x}^{\prime \prime}\right\|<\beta\right\}
$$

and for $\ell \in \mathbb{Z}^{d}$, let $\gamma_{\ell}(x)$ be the circle

$$
\gamma_{\ell}(x)=\left\{z \in \mathbb{C},\left|z-\ell_{x}^{\perp}\right|=c_{1} / 4\right\}
$$

where $c_{1}$ is the constant of Lemma 3.2 .

Now, we fixe $\beta, 0<\beta \ll c_{1} / 4$. Then for any $x$ and $\ell \in \Sigma_{\beta}(x)$, one has $\left|\lambda_{ \pm, \ell}^{0}(x)-\ell_{x}^{\perp}\right| \leq \beta \ll c_{1} / 4$, so the eigenvalues $\lambda_{ \pm, \ell}^{0}(x)$ are the only ones inside the circle $\gamma_{\ell}(x)$. By Lemma 3.3, one gets

$$
\left\{\begin{array}{l}
\left(\lambda-\mathbb{E}_{0}(x)\right)^{-1} \in \mathcal{A}^{0} \\
\left\|\left(\lambda-\mathbb{E}_{0}(x)\right)^{-1} ; \mathcal{H}^{0} \rightarrow \mathcal{H}^{0}\right\| \leq M
\end{array}\right\} \quad \forall \lambda \in \bigcup_{\ell \in \Sigma_{\beta}(x)} \gamma_{\ell}(x)
$$

We then apply Lemma A.1: $q$ vanishes on $\xi^{\prime}=0, \tau=0$ and $\theta$ is supported in $\left|\xi^{\prime}\right|+|\tau| \leq \alpha_{0}$. Therefore, if $\alpha_{0}$ is small enough, the resolvant $\left(\lambda-\mathbb{E}\left(t, x, \tau, \xi^{\prime}\right)\right)^{-1}$ exist for any $\left(t, x, \tau, \xi^{\prime}, \lambda\right)$ for $\lambda \in \bigcup_{\ell \in \Sigma_{\beta}(x)} \gamma_{\ell}(x)$. Obviously, 
one has

$$
\frac{1}{2 i \pi} \int_{\gamma_{\ell}(x)}\left(\lambda-\mathbb{E}_{0}(x)\right)^{-1} d \lambda\left(\sum_{k} z_{k} e^{i k y}\right)=z_{\ell} e^{i \ell y} .
$$

We choose $\psi \in C_{0}^{\infty}(]-1,1[)$ equal to 1 on $[-1 / 2,1 / 2]$ and we define $\operatorname{pr}_{0}(x), \operatorname{pr}\left(t, x, \tau, \xi^{\prime}\right)$ by the formulas

$$
\begin{gathered}
\operatorname{pr}_{0}(x)\left[\sum_{\ell} z_{\ell} e^{i \ell y}\right]=\sum_{\ell} \psi\left(\frac{\left\|\ell_{x}^{\prime \prime}\right\|^{2}}{\beta^{2}}\right) z_{\ell} e^{i \ell y} \\
\operatorname{pr}\left(t, x, \tau, \xi^{\prime}\right)=\sum_{\ell} \psi\left(\frac{\left\|\ell_{x}^{\prime \prime}\right\|^{2}}{\beta^{2}}\right) \frac{1}{2 i \pi} \int_{\gamma_{\ell}(x)}\left(\lambda-\mathbb{E}\left(t, x, \tau, \xi^{\prime}\right)\right)^{-1} d \lambda .
\end{gathered}
$$

The next lemma shows that $p r$ is well defined.

Lemma 3.4. There exists a $2 \times 2$ matrix $\delta p r\left(t, x, \tau, \xi^{\prime}\right)$ with entries in $\mathcal{S}^{t, 0}$, such that

$$
\left\{\begin{array}{l}
p r=p r_{0}+\delta p r \\
\delta p r_{\mid \xi^{\prime}=0, \tau=0}=0
\end{array}\right.
$$

Proof. See Appendix B.

Let $\varphi(t, x) \in C_{0}^{\infty}(U)$ equal to 1 near $\left(t_{0}, x_{0}^{\prime}\right)$. We next define $Q_{0}(t, x)$ and $Q\left(t, x, \tau, \xi^{\prime}\right)$ by the formulas, where $\left\langle\ell_{x}^{\perp}\right\rangle=\sqrt{1+\left|\ell_{x}^{\perp}\right|^{2}}$, and $\sigma=2\left|s_{0}\right|+2$

$$
\begin{gathered}
Q_{0}(t, x)\left[\Sigma z_{\ell} e^{i \ell y}\right]=\varphi \sum_{\ell} \psi\left(\frac{\left\|\ell_{x}^{\prime \prime}\right\|^{2}}{4 \beta^{2}}\right) \frac{1}{\left\langle\ell_{x}^{\perp}\right\rangle^{\sigma}}\left(\begin{array}{cc}
0 & -\left\langle\ell_{x}^{\prime \prime}\right\rangle \\
\frac{\left\|\ell_{x}^{\prime \prime}\right\|^{2}}{\left\langle\ell_{x}^{\prime \prime}\right\rangle} & 0
\end{array}\right)\left(z_{\ell}\right) e^{i \ell y} \\
Q\left(t, x, \tau, \xi^{\prime}\right)=\varphi \underset{\ell}{\sum_{\ell} \psi}\left(\frac{\left\|\ell_{x}^{\prime \prime}\right\|^{2}}{4 \beta^{2}}\right) \frac{1}{\left\langle\ell_{x}^{\perp}\right\rangle^{\sigma}} \frac{1}{2 i \pi} \int_{\gamma_{\ell}(x)}\left(\lambda-\mathbb{E}\left(t, x, \tau, \xi^{\prime}\right)\right)^{-1}\left(\mathbb{E}\left(t, x, \tau, \xi^{\prime}\right)-\ell_{x}^{\perp}\right) d \lambda .
\end{gathered}
$$

Lemma 3.5. There exist a $2 \times 2$ matrix $\delta Q\left(t, x, \tau, \xi^{\prime}\right)$ with entries in $\mathcal{S}^{t,-\sigma}$, such that

$$
\left\{\begin{array}{l}
Q=Q_{0}+\delta Q \\
\delta Q_{\mid \xi^{\prime}=0, \tau=0}=0
\end{array}\right.
$$

Proof. See Appendix B.

We then define $F^{\varepsilon, \mathbb{R}}$ and $F^{\varepsilon, I}$ by

$$
\left\{\begin{array}{l}
F^{\varepsilon, \mathbb{R}}=O p(p r) F^{\varepsilon} \\
F^{\varepsilon, I}=F^{\varepsilon}-F^{\varepsilon, \mathbb{R}} .
\end{array}\right.
$$

The Lemmas 3.4, A.2, the estimates (3.23) and (3.5), and the assumption $\left\|u^{\varepsilon}\right\| \leq 1$ imply

$$
\sup _{\varepsilon}\left\|F^{\varepsilon, \mathbb{R}, I} ; L^{2}\left(U ; \mathcal{H}^{s_{0}}\right)\right\|<+\infty
$$




$$
\sup _{\varepsilon} \varepsilon^{1 / 2} \| T r_{0,1}\left(F^{\varepsilon, \mathbb{R}, I}\right) ; L^{2}\left(U_{0} ; H^{s_{0}}\left(\mathbb{T}^{d}\right) \|<+\infty .\right.
$$

Moreover, $F^{\varepsilon, I}$ satisfies the following elliptic estimate

Lemma 3.6. There exist $D\left(t, x^{\prime}, \tau, \xi^{\prime}\right) \in \mathcal{S}^{0}$ such that

$$
\sup _{\varepsilon} \varepsilon^{-1 / 2} \| \operatorname{Tr}_{1}\left(F^{\varepsilon, I}\right)-O p(D) \operatorname{Tr}_{0}\left(F^{\varepsilon, I}\right) ; L^{2}\left(U_{0} ; H^{s_{0}-1}\left(\mathbb{T}^{d}\right) \|<+\infty .\right.
$$

Proof. See Appendix B.

To simplify notations, for $A \in \mathcal{S}^{t, *}$ we define $\widetilde{A}$ by $\widetilde{A}=O p(A)$, and for $g^{\varepsilon}$, a family depending on $\varepsilon$ in a norm space $\mathrm{B}, g^{\varepsilon} \in \varepsilon^{\alpha} B$ means $\sup \varepsilon^{-\alpha}\left\|g^{\varepsilon} ; B\right\|<+\infty$. We denote also by $\delta$ various symbols in $\mathcal{S}^{0}$ such that $\delta_{\mid \xi^{\prime}=0, \tau=0}=0$. We first notice that $\operatorname{Tr}_{0}\left(F^{\varepsilon}\right)=0$ and $(3.44)$ imply $\operatorname{Tr}_{0}\left(\widetilde{p r}_{0}\left(F^{\varepsilon}\right)\right)=0$, so by Lemma 3.4 we get

$$
\operatorname{Tr}_{0}\left(F^{\varepsilon, \mathbb{R}}\right)=\tilde{\delta} T r_{1}\left(F^{\varepsilon, \mathbb{R}}+F^{\varepsilon, I}\right) .
$$

By Lemma 3.6, and the Lemmas A.2 and A.3 on the symbolic calculus, we deduce from (3.53)

$$
\operatorname{Tr}_{0}\left(F^{\varepsilon, \mathbb{R}}\right)+\tilde{\delta} \operatorname{Tr}_{1}\left(F^{\varepsilon, \mathbb{R}}\right)+\tilde{\delta} T r_{0}\left(F^{\varepsilon, I}\right) \in \varepsilon^{1 / 2} L^{2}\left(U_{0}, H^{s_{0}-1}\right) .
$$

We have $\operatorname{Tr}_{0}\left(F^{\varepsilon, I}\right)=-\operatorname{Tr}_{0}\left(F^{\varepsilon, \mathbb{R}}\right)$, so (3.54) may be rewrite as a boundary condition for $F^{\varepsilon, \mathbb{R}}$

$$
(1-\tilde{\delta}) \operatorname{Tr}_{0}\left(F^{\varepsilon, \mathbb{R}}\right)+\tilde{\delta} \operatorname{Tr}_{1}\left(F^{\varepsilon, \mathbb{R}}\right) \in \varepsilon^{1 / 2} L^{2}\left(U_{0}, H^{s_{0}-1}\right) .
$$

By Lemma 3.1, $F^{\varepsilon, \mathbb{R}}$ satisfy the equation

$$
\left\{\begin{array}{l}
\frac{\varepsilon}{i} \partial_{x_{d}} F^{\varepsilon, \mathbb{R}}+\widetilde{\mathbb{E}} F^{\varepsilon, \mathbb{R}}=G^{\varepsilon, \mathbb{R}} \\
G^{\varepsilon, \mathbb{R}}=\widetilde{p r}\left(G^{\varepsilon}\right)+[\widetilde{E}, \widetilde{p r}] F^{\varepsilon}+\frac{\varepsilon}{i}\left(\partial_{x_{d}} \widetilde{p r}\right) F^{\varepsilon} .
\end{array}\right.
$$

By construction, we have $[\mathbb{E}, p r] \equiv 0$, so by Lemma A.3 $[\widetilde{\mathbb{E}}, \widetilde{p r}] \in \varepsilon \mathcal{S}^{t, 2}$ and from $(3.23,3.26)$ and Lemma A.2 we deduce

$$
G^{\varepsilon, \mathbb{R}} \in \varepsilon L^{2}\left(U, \mathcal{H}^{s_{0}-2}\right) .
$$

For $u(x, y) \in L^{2}\left(U, \mathcal{H}^{s}\right), v(x, y) \in L^{2}\left(U, \mathcal{H}^{-s}\right)$ let $\langle u \mid v\rangle$ be the duality

$$
\langle u \mid v\rangle=\int_{U}\left(\int_{\mathbb{T}^{d}} u(x, y) \bar{v}(x, y) d y\right) d x
$$

and let us define $J: L^{2}\left(U, \mathcal{H}^{s}\right) \rightarrow L^{2}\left(U, \mathcal{H}^{s}\right)$ by

$$
J\left(\begin{array}{l}
u_{0}(x, y) \\
u_{1}(x, y)
\end{array}\right)=\left(\begin{array}{l}
u_{1}(x, y) \\
u_{0}(x, y)
\end{array}\right) .
$$

By the choice $\sigma=2\left|s_{0}\right|+2$ and Lemma 3.6 (A.2) we have

$$
\left\{\begin{array}{l}
J \widetilde{Q} F^{\varepsilon, \mathbb{R}} \in L^{2}\left(U ; \mathcal{H}^{\left|s_{0}\right|+2}\right) \\
J \widetilde{Q} F^{\varepsilon, \mathbb{R}} \text { is compactly supported in } U .
\end{array}\right.
$$


Multiplying (3.56) by $J \widetilde{Q} F^{\varepsilon, \mathbb{R}}$, we obtain (where $(\cdot \mid \cdot)$ is the duality on $x_{d}=0$ )

$$
\left\{\begin{aligned}
&\left\langle G^{\varepsilon, \mathbb{R}} \mid J \widetilde{Q} F^{\varepsilon, \mathbb{R}}\right\rangle=\left\langle\frac{\varepsilon}{i} \partial_{x_{d}} F^{\varepsilon, \mathbb{R}} \mid J \widetilde{Q} F^{\varepsilon, \mathbb{R}}\right\rangle+\left\langle J \widetilde{\mathbb{E}} F^{\varepsilon, \mathbb{R}} \mid \widetilde{Q} F^{\varepsilon, \mathbb{R}}\right\rangle \\
&=-\frac{\varepsilon}{i}\left(\left.F^{\varepsilon, \mathbb{R}}\right|_{x_{d}=0}\left|J \widetilde{Q} F^{\varepsilon, \mathbb{R}}\right|_{x_{d}=0}\right)+\mathcal{J}_{1}+\mathcal{J}_{2}+\mathcal{J}_{3} \\
& \mathcal{J}_{1}=\left\langle F^{\varepsilon, \mathbb{R}} \mid\left\{(J \widetilde{\mathbb{E}})^{*}-J \widetilde{\mathbb{E}}\right\} \widetilde{Q} F^{\varepsilon, \mathbb{R}}\right\rangle \\
& \mathcal{J}_{2}=\left\langle J F^{\varepsilon, \mathbb{R}} \mid\left[\frac{\varepsilon}{i} \partial_{x_{d}}+\widetilde{\mathbb{E}}, \widetilde{Q}\right] F^{\varepsilon, \mathbb{R}}\right\rangle \\
& \mathcal{J}_{3}=\left\langle J F^{\varepsilon, \mathbb{R}} \mid \widetilde{Q} G^{\varepsilon, \mathbb{R}}\right\rangle .
\end{aligned}\right.
$$

By (3.57) and (3.60), both $\left|\left\langle G^{\varepsilon, \mathbb{R}} \mid J \widetilde{Q} F^{\varepsilon, \mathbb{R}}\right\rangle\right|$ and $\mathcal{J}_{3}$ are $\mathcal{O}(\varepsilon)$. By construction of $Q$ (see (3.50)) we have $[\mathbb{E}, Q] \equiv 0$ so by Lemma A.3, we get $\mathcal{J}_{2} \in \mathcal{O}(\varepsilon)$. Finally, we have

so we obtain

$$
\begin{gathered}
J \mathbb{E}=J \mathbb{E}_{0}+\left(\begin{array}{cc}
q \theta & 0 \\
0 & 0
\end{array}\right) ; \\
J \mathbb{E}_{0}\left(\sum_{\ell} z_{\ell} e^{i \ell y}\right)=\sum_{\ell}\left(\begin{array}{cc}
\frac{\left\|\ell_{x}^{\prime \prime}\right\|^{2}}{\left\langle\ell_{x}^{\prime \prime}\right\rangle} & \ell_{x}^{\perp} \\
\ell_{x}^{\perp} & -\left\langle\ell_{x}^{\prime \prime}\right\rangle
\end{array}\right)\left(z_{\ell}\right) e^{i \ell y}
\end{gathered}
$$

$$
(J \widetilde{\mathbb{E}})^{*}-(J \widetilde{E})=\left(\begin{array}{ccc}
(\widetilde{q \theta})^{*}-q \theta & 0 \\
0 & 0
\end{array}\right) .
$$

By formula (3.32) and if we choose $\theta\left(t, x, \tau, \xi^{\prime}\right)$ real, $q \theta$ is self adjoint, so from Lemma A.4 we deduce $\mathcal{J}_{1} \in \mathcal{O}(\varepsilon)$. Summing up, we have thus

$$
\sup _{\varepsilon}\left|\left(\left.F^{\varepsilon, \mathbb{R}}\right|_{x_{d}=0}\left|J \widetilde{Q} F^{\varepsilon, \mathbb{R}}\right|_{x_{d}=0}\right)\right|<+\infty
$$

We now remark that if $\delta_{1} \in \mathcal{S}^{0}$ vanishes on $\xi^{\prime}=0, \tau=0$, there exist $\delta_{2} \in \mathcal{S}^{0}$, vanishing on $\xi^{\prime}=\tau=0$ such that $\left(1+\delta_{2}\right)\left(1-\delta_{1}\right)=1-p$, where $p\left(t, x, \tau, \xi^{\prime}\right) \in \mathcal{S}^{0}$, is supported in $c_{0} \leq|\tau|+\left|\xi^{\prime}\right| \leq 1 / c_{0}$ for some $c_{0}>0$. Decreasing $\alpha_{0}$, hence $W_{0}$, if necessary, we then will have $\tilde{p} T r_{0,1}\left(F^{\varepsilon, \mathbb{R}}\right) \in \varepsilon^{1 / 2} L^{2}\left(U_{0}, H^{s_{0}-1}\right)$. Using once more Lemma A.3, we can thus rewrite the boundary condition (3.55) on the form

$$
\operatorname{Tr}_{0}\left(F^{\varepsilon, \mathbb{R}}\right)-\tilde{\delta} T r_{1}\left(F^{\varepsilon, \mathbb{R}}\right) \in \varepsilon^{1 / 2} L^{2}\left(U_{0}, H^{s_{0}-1}\right)
$$

Let $Q=\left(\begin{array}{ll}Q^{1} & Q^{2} \\ Q^{3} & Q^{4}\end{array}\right)$; inserting (3.64) in (3.63) and taking in account the a priori estimate (3.52) we get

$$
\left\{\begin{array}{l}
\sup _{\varepsilon}\left|\left(\operatorname{Tr}_{1}\left(F^{\varepsilon, \mathbb{R}}\right) \mid \widetilde{A} \operatorname{Tr}_{1}\left(F^{\varepsilon, \mathbb{R}}\right)\right)\right|<+\infty \\
A=Q^{2}+Q^{1} \delta+\delta^{*} Q^{3} \delta+\delta^{*} Q^{4}
\end{array}\right.
$$

Let (we use Lem. 3.4 for the second equality)

$$
F_{0}^{\varepsilon, \mathbb{R}}=\widetilde{p r}_{0}\left(F^{\varepsilon}\right)=F^{\varepsilon, \mathbb{R}}+\delta\left(F^{\varepsilon, \mathbb{R}}+F^{\varepsilon, I}\right) .
$$


We know already that $\operatorname{Tr}_{0}\left(F^{\varepsilon, \mathbb{R}}\right), \operatorname{Tr}_{0}\left(F^{\varepsilon, I}\right)$ and $\operatorname{Tr}_{1}\left(F^{\varepsilon, I}\right)$ are of the form $\tilde{\delta} \operatorname{Tr}_{1}\left(F^{\varepsilon, \mathbb{R}}\right)+\varepsilon^{1 / 2} L^{2}\left(U_{0}, H^{s_{0}-1}\right)$ so we get from (3.66)

$$
\operatorname{Tr}_{1}\left(F_{0}^{\varepsilon, \mathbb{R}}\right)=(1+\delta) \operatorname{Tr}_{1}\left(F^{\varepsilon, \mathbb{R}}\right)+\varepsilon^{1 / 2} L^{2}\left(U_{0}, H^{s_{0}-1}\right) .
$$

Decreasing $\alpha_{0}$ if necessary we get as above

$$
\operatorname{Tr}_{1}\left(F^{\varepsilon, \mathbb{R}}\right)=(1+\delta) \operatorname{Tr}_{1}\left(F_{0}^{\varepsilon, \mathbb{R}}\right)+\varepsilon^{1 / 2} L^{2}\left(U_{0}, H^{s_{0}-1}\right) .
$$

Therefore (3.65) and (3.68) imply

$$
\left\{\begin{array}{l}
\sup _{\varepsilon}\left|\left(\operatorname{Tr}_{1}\left(F_{0}^{\varepsilon, \mathbb{R}}\right) \mid \widetilde{A}_{0} \operatorname{Tr}_{1}\left(F_{0}^{\varepsilon, \mathbb{R}}\right)\right)\right|<+\infty \\
A_{0}=Q_{0}^{2}+\delta^{*} A_{1}+A_{2} \delta
\end{array}\right.
$$

with $A_{1}, A_{2} \in \mathcal{S}^{+\sigma}$.

By $(3.66,3.44), \operatorname{Tr}_{1}\left(F_{0}^{\varepsilon, \mathbb{R}}\right)$ is of the form

$$
\operatorname{Tr}_{1}\left(F_{0}^{\varepsilon, \mathbb{R}}\right)=\sum_{\left\|\ell_{\left(x^{\prime}, 0\right)}^{\prime \prime}\right\| \leq \beta} z_{\ell}\left(t, x^{\prime}\right) e^{i \ell y}
$$

and we may assume that the functions $z_{\ell}\left(t, x^{\prime}\right)$ are supported in $\{\varphi \equiv 1\}$.

For $\left\|\ell_{x}^{\prime \prime}\right\| \leq \beta$ we have $\psi\left(\frac{\left\|\ell_{x}^{\prime \prime}\right\|^{2}}{4 \beta^{2}}\right)=1$, and $\frac{\left|\left\langle\ell_{x}^{\prime \prime}\right\rangle\right|}{\left\langle\ell_{x}^{\frac{1}{x}}\right\rangle^{\sigma}} \sim(1+|\ell|)^{-\left(2\left|s_{0}\right|+2\right)}$; from (3.47) we therefore get for some $C_{0}>0$

$$
\left|\left(\operatorname{Tr}_{1}\left(F_{0}^{\varepsilon, \mathbb{R}}\right) \mid Q_{0}^{2} \operatorname{Tr}_{1}\left(F_{0}^{\varepsilon, \mathbb{R}}\right)\right)\right| \geq C_{0}\left\|\operatorname{Tr}_{1}\left(F_{0}^{\varepsilon, \mathbb{R}}\right) ; L^{2}\left(U_{0}, H^{s_{0}-1}\right)\right\|^{2} .
$$

We now remark that in (3.69), we may replace any $\delta\left(t, x^{\prime}, \tau, \xi^{\prime}\right)$ term by $\chi\left(\left(\tau, \xi^{\prime}\right) / \alpha_{0}\right) \delta\left(t, x^{\prime}, \tau, \xi^{\prime}\right)$, with $\chi \in C_{0}^{\infty}$ equal to 1 in the unit ball, and

$$
\chi\left(\left(\tau, \xi^{\prime}\right) / \alpha_{0}\right) \delta=\sum_{j=1}^{d-1} \chi\left(\left(\tau, \xi^{\prime}\right) / \alpha_{0}\right) \xi_{j}^{\prime} b_{j}+\chi\left(\left(\tau, \xi^{\prime}\right) / \alpha_{0}\right) \tau b_{0}
$$

where $b_{*} \in \mathcal{S}^{0}$ so we have, for some $C_{1}>0$

$$
\left|\left(\operatorname{Tr}_{1}\left(F_{0}^{\varepsilon, \mathbb{R}}\right) \mid\left(\widetilde{\delta A_{0}}\right) \operatorname{Tr}_{1}\left(F_{0}^{\varepsilon, \mathbb{R}}\right)\right)\right| \leq C_{1} \alpha_{0}\left\|\operatorname{Tr}_{1}\left(F_{0}^{\varepsilon, \mathbb{R}}\right) ; L^{2}\left(U_{0}, H^{s_{0}-1}\right)\right\|^{2}
$$

From $(3.69,3.71,3.72)$ we get, for $\alpha_{0}$ small,

$$
\sup _{\varepsilon}\left\|\operatorname{Tr}_{1}\left(F_{0}^{\varepsilon, \mathbb{R}}\right) ; L^{2}\left(U_{0} ; H^{s_{0}-1}\right)\right\|<+\infty
$$

so by (3.68), the same estimate holds true for $\operatorname{Tr}_{1}\left(F^{\varepsilon, \mathbb{R}}\right)$, hence also for

$$
\operatorname{Tr}_{1}\left(F^{\varepsilon, I}\right)=\tilde{\delta} \operatorname{Tr}_{1}\left(F^{\varepsilon, \mathbb{R}}\right)+\varepsilon^{1 / 2} L^{2}\left(U_{0}, H^{s_{0}-1}\right) .
$$

Thus we have

$$
\sup _{\varepsilon}\left\|\operatorname{Tr}_{1}\left(F^{\varepsilon}\right) ; L^{2}\left(U_{0}, H^{s_{0}-1}\right)\right\|<\infty .
$$

This concludes the proof of Theorem 2 . 


\section{Propagation estimate}

This section is devoted to the proof of Proposition 0.3. We fix a zero order o.p.d. $Q\left(\varepsilon, t, x, \varepsilon \partial_{x^{\prime}}, \varepsilon \partial_{t}\right)$ equal to $I d$ near $K$, with essential support in $W_{1}$ and we argue by contradiction. If $(0.50)$ is untrue, there exist sequences $\varepsilon_{k} \rightarrow 0, \gamma_{k} \rightarrow 0 \quad h_{k} \rightarrow 0, h_{k} \geq \varepsilon_{k} / \gamma_{k}$, and $u^{k} \in I_{h_{k}}^{\varepsilon_{k}}$ such that

$$
\left\{\begin{array}{l}
\left\|u^{k}\right\|=1 \\
\frac{1}{k}\left\|Q \Gamma_{0}\left(u^{k}\right)\right\|_{L^{2}\left(X_{T_{0}}\right)}^{2} \geq\left[\left\|\Gamma_{0}\left(u^{k}\right)_{\mid x_{d}=0}\right\|_{L^{2}\left(X_{T_{0}} \cap x_{d}=0\right)}^{2}+\left\|u^{k}\right\|_{L^{2}\left(\left(0, T_{0}\right) \times V\right)}^{2}\right] .
\end{array}\right.
$$

In particular the right hand side of the second line in (4.1) goes to zero.

Let $\mathcal{L}$ and $\left[\begin{array}{l}g_{0} \\ g_{1}\end{array}\right]$ be defined by the formula $(0.51)$ with $u^{\varepsilon}=u^{k}$. We have

$$
L \sim \sum_{n}\left(\frac{\varepsilon}{i}\right)^{n} L^{n}, L^{n}=\left(\begin{array}{ll}
L_{1}^{n} & L_{2}^{n} \\
L_{3}^{n} & L_{4}^{n}
\end{array}\right)
$$

so we get

$$
\mathcal{L} \sim\left(\begin{array}{cc}
h / \varepsilon L_{1}^{0} & L_{2}^{0} \\
h^{2} / \varepsilon^{2} L_{3}^{0} & h / \varepsilon L_{4}^{0}
\end{array}\right)+\frac{h}{i}\left(\begin{array}{cc}
L_{1}^{1} & \varepsilon / h L_{2}^{1} \\
h / \varepsilon L_{3}^{1} & L_{4}^{1}
\end{array}\right)+\sum_{n \geq 2}\left(\frac{h}{i}\right)^{n}\left(\frac{\varepsilon}{h}\right)^{n-1}\left(\begin{array}{cc}
L_{1}^{n} & \varepsilon / h L_{2}^{n} \\
h / \varepsilon L_{3}^{n} & L_{4}^{n}
\end{array}\right)
$$

By Lemma 2.1, i) $\frac{h}{\varepsilon} L_{3}^{1}$ is a smooth function of $x, \xi^{\prime}=\frac{h}{i} \partial_{x^{\prime}}, \tau=\frac{h}{i} \partial_{t}$, defined for $\frac{h}{\varepsilon}\left(\left|\xi^{\prime}\right|+|\tau|\right)$ small. Therefore, $\mathcal{L}\left(h, x, \frac{h}{i} \partial_{x^{\prime}}, \frac{h}{i} \partial_{t}\right)$ is a $h$-o.p.d. defined for $\frac{h}{\varepsilon}\left(\left|\xi^{\prime}\right|+|\tau|\right)$ small, with asymptotic development

$$
\mathcal{L} \sim \sum_{n \geq 0}\left(\frac{h}{i}\right)^{n} \mathcal{L}^{n}
$$

and by Lemma 2.1 , ii) we get

$$
\mathcal{L}_{0}=\left(\begin{array}{cc}
a_{0}^{-1}(x) a_{1}\left(x, i \xi^{\prime}\right) & -a_{0}^{-1}(x) \\
a_{2}\left(x, i \xi^{\prime}\right)+\underline{\hat{\rho}}(x) \tau^{2} & 0
\end{array}\right)+0\left(\left(\frac{\varepsilon}{h}\right)^{2} \tau^{4}\right)
$$

Let $\underline{u}^{k}$ be the extension of $u^{k}$ by zero outside $\Omega$. Let $\mu$ be a $h$-semiclassical measure associated to $\left\{\underline{u}^{k}\right\}$ (see [8]). Let $\underline{g}_{0}^{k}$ the extension of $g_{0}^{k}=\Gamma_{0}\left(u^{k}\right)$ by zero on $x_{d}<0$ and let $\nu$ be a $h$-semiclassical measure associated to $\underline{g}_{0}^{k}$. Using (2.45) and $\lim _{k \rightarrow \infty} \varepsilon_{k} / h_{k}=0$ we get

$$
\nu=\chi_{0}^{2}\left(t, x ; \xi^{\prime}=0, \tau=0\right) \mu \quad(\text { for } x \in \partial \Omega \times]-r_{0}, r_{0}[)
$$

We have $u^{k} \in I_{h_{k}}^{\varepsilon_{k}}$ so we know that $\mu$ is supported in $|\tau| \in[0.9,2.1]$; moreover, by the proof of Proposition 1 Section 2, the support of $\mu_{\mid \Omega}$ is contained in the set $\underline{\rho}(x) \tau^{2}-\|\xi\|^{2}=0$, and $\mu \mid \Omega$ propagates on the bicharacteristic flow of $\underline{\rho}(x) \tau^{2}-\|\xi\|^{2}$. Let $g^{k}=\Gamma\left(u^{k}\right)$, and let $\bar{A}\left(h, t, x, h \partial_{x^{\prime}}, h \partial_{t}\right)$ be any $h$-o.p.d. compactly supported in $T^{*}\left(X_{T_{0}}\right)$. Using $(0.41,0.42)$ and $\lim _{k \rightarrow \infty} \varepsilon_{k} / h_{k}=0$ we get with $h=h_{k}$

$$
A\left[\left(h \frac{\partial}{\partial x_{d}}+\mathcal{L}\right) g^{k}\right] \in \mathcal{O}\left(h^{\infty} L^{2}\right)
$$


Writing $\mathcal{L}=\left(\begin{array}{ll}\mathcal{L}_{1} & \mathcal{L}_{2} \\ \mathcal{L}_{3} & \mathcal{L}_{4}\end{array}\right)$, we observe that the principal symbol of $\mathcal{L}_{1}$ vanishes near $x_{d}=0$. Using $(4.1,4.7)$ we get that $g_{0}^{k}$ satisfies near the boundary the second order tangential $h$-pseudo differential equation, with $h=h_{k}$

$$
\left\{\begin{array}{l}
A\left[\left(h \partial_{x_{d}}\right)^{2} g_{0}^{k}+\left(R_{2}+h R_{1} h \frac{\partial}{\partial x_{d}}\right) g_{0}^{k}\right] \in \mathcal{O}\left(h^{\infty} L^{2}\right) \\
\lim _{k \rightarrow \infty}\left\|\left.g_{0}^{k}\right|_{x_{d}=0}\right\|_{L^{2}}=0
\end{array}\right.
$$

where $R_{1,2}$ are $h$-tangential o.p.d. defined for $\frac{h}{\varepsilon}\left(\left|\xi^{\prime}\right|+|\tau|\right)$ small, and the principal symbol of $R_{2}, R_{2}^{0}$ is given by

$$
R_{2}^{0}=a_{2}\left(x, i \xi^{\prime}\right)+\underline{\rho}(x) \tau^{2}+0\left(\left(\frac{\varepsilon}{h}\right)^{2} \tau^{4}\right)
$$

We can now use the propagation theorem at the boundary for second order Dirichlet problem (see [8] for the localization and propagation at hyperbolic point and [10], Append. or [3], Th. 1 for the propagation result near the glancing set; here we view $\gamma_{k}=\varepsilon_{k} / h_{k}$ as a small parameter in equation (4.8), and we notice that the proof of the propagation theorem allows this additional parameter going to zero). We get that the support of $\nu$ is contained in the set $\rho(x) \tau^{2}-\|\xi\|^{2}=0$, and that the support of $\nu$ propagates along the generalized bicharacteristic flow of $\underline{\rho}(x) \tau^{2}-\|\xi\|^{2}$; but (4.1) implies $\mu_{\mid] 0, T_{0}[\times V} \equiv 0$, so from (0.9) and (4.6) we get $\mu_{\mid t \in] T_{0} / 2-\alpha, T_{0} / 2+\alpha[} \equiv 0$ for $\alpha$ small. This is in contradiction with $\left\|u^{k}\right\|=1$ by $(2.41)$.

\section{A. Semi-Classical O.P.D. With OPERATOR VAlues}

\section{A.1. Classical calculus}

We recall here some classical properties of semi-classical tangential pseudo differential operators. Let $Z=\mathbb{R}_{z}^{p} \times\left[0, r_{0}\right]_{x_{d}}$ and $H_{1}, H_{2}$ two separable Hilbert spaces.

We denote by $S_{Z}^{t}\left(H_{1} \rightarrow H_{2}\right)$ the vector space of functions $q\left(\varepsilon, z, \zeta, x_{d}\right)$ defined for $\left.\left.\varepsilon \in\right] 0, \varepsilon_{0}\right]\left(\varepsilon_{0}\right.$ small $)$ smooth in $(z, \zeta) \in T^{*} \mathbb{R}_{z}^{p}, x_{d} \in\left[0, r_{0}\right]$, compactly supported in $z$, with values in bounded operators from $H_{1}$ to $H_{2}$ which satisfies the estimates

$$
\begin{aligned}
& \forall \alpha, k \exists C_{\alpha, k} \forall \varepsilon, z, \zeta, x_{d} \\
& \left\|(1+|\zeta|)^{k} \partial_{z, \zeta, x_{d}}^{\alpha} q\left(\varepsilon, z, \zeta, x_{d}\right) ; H_{1} \rightarrow H_{2}\right\| \leq C_{\alpha, k}
\end{aligned}
$$

and admitting classical asymptotic expansions in $\varepsilon$

$$
q \sim \sum_{n=0}^{\infty}\left(\frac{\varepsilon}{i}\right)^{n} q_{n}\left(z, \zeta, x_{d}\right) \Leftrightarrow \forall N \quad q-\sum_{n<N}\left(\frac{\varepsilon}{i}\right)^{n} q_{n} \in \varepsilon^{N} S_{Z}^{t} .
$$

For $f\left(z, x_{d}\right) \in L^{2}\left(Z, H_{1}\right)$ with compact support in $z$, the Fourier transform $\hat{f}_{\varepsilon}\left(\zeta, x_{d}\right)$ is defined by

$$
\hat{f}_{\varepsilon}\left(\zeta, x_{d}\right)=\int e^{-i z \zeta / \varepsilon} f\left(z, x_{d}\right) d z \in L^{2}\left(\mathbb{R}_{\zeta}^{p} \times\left[0, x_{d}\right], H_{1}\right)
$$

and for $q \in S_{Z}^{t}\left(H_{1}, H_{2}\right), O p(q)(f)$ is defined by

$$
O p(q)(f)\left(\varepsilon, z, x_{d}\right)=(2 \pi \varepsilon)^{-p} \int e^{i z \zeta / \varepsilon} q\left(\varepsilon, z, \zeta, x_{d}\right)\left[\hat{f}_{\varepsilon}\left(\zeta, x_{d}\right)\right] d \zeta .
$$


We define the set $\mathcal{E}_{Z}^{t}\left(H_{1} \rightarrow H_{2}\right)$ of tangential pseudo differential operators from $L^{2}\left(Z, H_{1}\right)$ to $L^{2}\left(Z, H_{2}\right)$ by

$$
\left\{\begin{array}{l}
Q=Q_{\varepsilon} \in \mathcal{E}_{Z}^{t}\left(H_{1} \rightarrow H_{2}\right) \text { iff there exist } \varphi(z) \in C_{0}^{\infty}\left(\mathbb{R}_{z}^{p}\right) \\
\text { and } \tilde{q} \in S_{Z}^{t}\left(H_{1} \rightarrow H_{2}\right) \text { such that } \\
Q_{\varepsilon}(f)(z)=O p(\tilde{q})[\varphi(z) f] \quad \forall f \in L^{2}\left(Z, H_{1}\right) .
\end{array}\right.
$$

For $Q \in \mathcal{E}_{Z}^{t}\left(H_{1} \rightarrow H_{2}\right)$, one has $Q=O p(q)$ with

$$
q\left(\varepsilon, z, \zeta, x_{d}\right)=(2 \pi)^{-p} \int e^{-i t \theta} \tilde{q}\left(\varepsilon, z, \zeta+\varepsilon \theta, x_{d}\right) \varphi(z+t) d t d \theta
$$

and $Q$ is bounded on $L^{2}$, i.e.

$$
\exists C \quad \forall \varepsilon \quad\left\|Q_{\varepsilon}(f) ; L^{2}\left(Z, H_{2}\right)\right\| \leq C\left\|f ; L^{2}\left(Z, H_{1}\right)\right\| .
$$

For $Q_{1}=O p\left(q_{1}\right) \in \mathcal{E}_{Z}^{t}\left(H_{1} \rightarrow H_{2}\right)$ and $Q_{2}=O p\left(q_{2}\right) \in \mathcal{E}_{Z}^{t}\left(H_{2} \rightarrow H_{3}\right)$, one has $Q_{1} \circ Q_{2}=O p(q)=Q \in \mathcal{E}_{Z}^{t}\left(H_{1}\right.$ $\left.\rightarrow H_{3}\right)$ with

$$
q\left(\varepsilon, z, \zeta, x_{d}\right)=(2 \pi)^{-p} \int e^{-i t \theta} q_{1}\left(\varepsilon, z, \zeta+\varepsilon \theta, x_{d}\right) \circ q_{2}\left(\varepsilon, z+t, \zeta, x_{d}\right) d t d \theta
$$

and the asymptotic expansion of $q$ is given by the rule

$$
q \sim \sum_{\alpha}\left(\frac{\varepsilon}{i}\right)^{|\alpha|} \frac{1}{\alpha !} \partial_{\zeta}^{\alpha} q_{1} \circ \partial_{z}^{\alpha} q_{2}
$$

The set of operators $\mathcal{E}_{Z}^{t}\left(H_{1} \rightarrow H_{2}\right)$ is free of coordinates, i.e., if $z \mapsto \phi(z)$ is a smooth diffeomorphism of $\mathbb{R}_{z}^{p}$, and $Q \in \mathcal{E}_{Z}^{t}$, then $\phi \circ Q \circ \phi^{-1} \in \mathcal{E}_{Z}^{t}$. Thus, in the definition of $\mathcal{E}_{Z}^{t}$, we can replace $\mathbb{R}_{z}^{p}$ by a smooth manifold $M$. For $Q=O p(q) \in \mathcal{E}_{Z}^{t}\left(H_{1} \rightarrow H_{2}\right)$ its principal symbol, $q_{0}\left(z, \zeta, x_{d}\right)$ is then defined as a smooth function of $\left(z, \zeta, x_{d}\right) \in T^{*} M \times\left[0, r_{0}\right]$, with values in bounded operators from $H_{1}$ to $H_{2}$. For $Q=O p(q) \in \mathcal{E}_{Z}^{t}$, the essential support of $Q S E(Q)$ is the closed subset of $T^{*} M \times\left[0, r_{0}\right]$ defined by

$$
\left\{\begin{array}{l}
\rho_{0}=\left(z_{0}, \zeta_{0}, x_{d, 0}\right) \notin S E(Q) \text { iff there exists a neighborhood } \\
W \text { of } \rho_{0} \text { such that } q_{\mid W} \sim 0 .
\end{array}\right.
$$

Let $K$ be a compact subset of $T^{*} M \times\left[0, r_{0}\right]$. One says that $Q_{1} \equiv Q_{2}$ near $K$ if $S E\left(Q_{1}-Q_{2}\right) \cap K=\phi$ and if $u: H_{1} \rightarrow H_{2}$ is bounded, $Q \equiv u$ near $K$ means $Q-\varphi\left(y, x_{d}\right) u \equiv 0$ for some $\varphi \in C_{0}^{\infty}\left(M \times\left[0, r_{0}\right]\right)$ equal to 1 near the projection of $K$ on $M \times\left[0, r_{0}\right]$. If $Q \equiv 0$ near $K$, for any scalar tangential o.p.d. $P \in \mathcal{E}_{Z}^{t}(\mathbb{C} \rightarrow \mathbb{C})$, such that $S E(P) \subset K$ one has

$$
\forall N, \exists C_{N} \| Q P \text { or } P Q ; L^{2}\left(Z, H_{1}\right) \rightarrow L^{2}\left(Z, H_{2}\right) \| \leq C_{N} \varepsilon^{N} .
$$

One says that $Q=O p(q) \in \mathcal{E}_{Z}^{t}$ is elliptic on $K$ if for any $\rho=\left(z, \zeta, x_{d}\right) \in K$, the principal symbol $q_{0}(\rho)$ is an isomorphism from $H_{1}$ onto $H_{2}$. In that case, there exist $E \in \mathcal{E}_{Z}^{t}\left(H_{2} \rightarrow H_{1}\right)$ with principal symbol $e_{0}$ equal to $q_{0}^{-1}$ near $K$ such that $E \circ Q \equiv I d_{H_{1}}$ and $Q \circ E \equiv I d_{H_{2}}$ near $K$.

\section{A.2. An exotic calculus}

Let $\mathbb{T}_{Y}^{d}$ be the $d$-dimensional torus, and for $s \in \mathbb{R}, H^{s}$ the usual Sobolev space

$$
H^{s}=\left\{\sum_{\ell \in \mathbb{Z}^{d}} a_{\ell} e^{i \ell y}, \sum_{\ell}\left(1+|\ell|^{2}\right)^{s}\left|a_{\ell}\right|^{2}<\infty\right\} .
$$


For any operator $A: \cap_{s} H^{s} \rightarrow \cup H^{s}$, we denote by $A_{\ell, k}$ the matrix coefficient

$$
A_{\ell, k}=\oint_{\mathbb{T}^{d}}\left(A e^{i k y}\right) \cdot e^{-i \ell y}
$$

For $m \in \mathbb{R}$, let $\mathcal{A}^{m}$ be the following class of operators on the torus

$$
\mathcal{A}^{m}=\left\{A ; \forall N, \exists C_{N} \quad\left|A_{\ell, k}\right| \leq C_{N} \frac{(1+|\ell|)^{m}}{(1+|\ell-k|)^{N}} \quad \forall \ell, k \in \mathbb{Z}^{d}\right\}
$$

One has $\mathcal{A}^{m} \circ \mathcal{A}^{m^{\prime}} \subset \mathcal{A}^{m+m^{\prime}}$, and for $A \in \mathcal{A}^{m}, A$ is bounded from $H^{s}$ to $H^{s-m}$ for any $s \in \mathbb{R}$. The identity

$$
\left[D_{j}, A\right]_{\ell, k}=\left(\ell_{j}-k_{j}\right) A_{\ell, k} \quad D_{j}=\frac{1}{i} \frac{\partial}{\partial y_{j}}
$$

shows that $\mathcal{A}^{0}$ is the class of bounded operators on $L^{2}=H^{0}$ such that all the commutators

$$
\left[D_{j_{1}},\left[D_{j_{2}}, \cdots\left[D_{j_{p}}, A\right] \cdots\right]\right]
$$

are bounded on $L^{2}$.As a consequence, we get

Lemma A.1. Let $A \in \mathcal{A}^{0}$, and $\delta<\left(\left\|A ; L^{2} \rightarrow L^{2}\right\|\right)^{-1}$. Then $(I d+\delta A)^{-1} \in \mathcal{A}^{0}$.

Proof. $B=(I d+\delta A)^{-1}$ is bounded on $L^{2}$, and all the commutators (A.14) for $B$ can be expressed in terms of commutators for $A$ by iteration of the formula

$$
\left[D_{j}, B\right]=-B \delta\left[D_{j}, A\right] B .
$$

Let $Z=\mathbb{R}_{z}^{p} \times\left[0, r_{0}\right]$.

We denote by $\mathcal{S}_{Z}^{t, m}$ the vector space of functions $A\left(\varepsilon, z, \zeta, x_{d}\right)$ defined for $\left.\left.\varepsilon \in\right] 0, \varepsilon_{0}\right]$ smooth in $(z, \zeta) \in$ $T^{*} \mathbb{R}_{z}^{p}, x_{d} \in\left[0, r_{0}\right]$, with values operators on the torus, which satisfy the estimates

$$
\left\{\begin{array}{l}
\forall \alpha, \beta, \gamma, N, \exists C, \quad \forall \varepsilon, \ell, k, z, \zeta, x_{d} \\
\left|(1+|\zeta|)^{\gamma} \partial_{z, x_{d}}^{\alpha} \partial_{\zeta}^{\beta} A_{\ell, k}\left(\varepsilon, z, \zeta, x_{d}\right)\right| \leq C \frac{(1+|\ell|)^{m+|\alpha|}}{(1+|\ell-k|)^{N}}
\end{array}\right.
$$

In other words, $A \in \mathcal{S}_{Z}^{t, m}$ means

$$
\forall \alpha, \beta, \gamma \quad(1+|\zeta|)^{\gamma} \partial_{z, x_{d}}^{\alpha} \partial_{\zeta}^{\beta} A \in \mathcal{A}^{m+|\alpha|}
$$

uniformly in $\varepsilon, z, \zeta, x_{d}$.

Leibniz formula implies

$$
\mathcal{S}_{Z}^{t, m} \circ \mathcal{S}_{Z}^{t, m^{\prime}} \subset \mathcal{S}_{Z}^{t, m+m^{\prime}}
$$

We denote by $\mathcal{B}_{Z}^{t, m}$ the class of operators

$$
\left\{\begin{array}{l}
O p(A) ; A \in \mathcal{S}_{Z}^{t, m} \\
O p(A)[f]\left(z, x_{d}\right)=(2 \pi \varepsilon)^{-p} \int e^{i z \zeta / \varepsilon} A\left(\varepsilon, z, \zeta, x_{d}\right)\left[\hat{f}_{\varepsilon}\left(\zeta, x_{d}\right)\right] d \zeta
\end{array}\right.
$$


where $f \in L^{2}\left(Z ; H^{s}\right)$ for some $s, Z=\mathbb{R}_{z}^{p} \times\left(0, r_{0}\right)_{x_{d}}$, and $\hat{f}_{\varepsilon}$ is the partial Fourier transform

$$
\hat{f}_{\varepsilon}\left(\zeta, x_{d}\right)=\int e^{-i z \zeta / \varepsilon} f\left(z, x_{d}\right) d z \in L^{2}\left(\mathbb{R}_{\zeta}^{p} \times\left(0, r_{0}\right) ; H^{s}\right) .
$$

Lemma A.2. For any $A \in \mathcal{S}_{Z}^{t, m}, O p(A)$ is bounded from $L^{2}\left(Z ; H^{s}\right)$ in $L^{2}\left(Z ; H^{s-m}\right)$ for any $s$, uniformly in $\left.\varepsilon \in] 0, \varepsilon_{0}\right]$.

Proof. To avoid the loose of derivative in $z$, we use the fact that $A$ is a Schwartz function in $\zeta$, so we can write, by Fourier inversion formula

$$
A\left(\varepsilon, z, \zeta, x_{d}\right)=(2 \pi)^{-p} \int e^{i \zeta \theta} B\left(\varepsilon, z, \theta, x_{d}\right) d \theta
$$

with $B \in \mathcal{S}_{Z}^{t, m}$; we obtain

$$
O p(A)(f)=(2 \pi)^{-p} \int B\left(\varepsilon, z, \theta, x_{d}\right)\left[f\left(z+\varepsilon \theta, x_{d}\right)\right] d \theta .
$$

The bounds (A.15) for $B$ (with $\alpha=\beta=0,|\gamma|=p+1$ ) imply

$$
\forall s, \exists C_{s} \sup _{\varepsilon}\left\|B(\varepsilon, \cdot, \theta, \cdot) ; L^{2}\left(Z ; H^{s}\right) \rightarrow L^{2}\left(Z ; H^{s-m}\right)\right\| \leq \frac{C_{s}}{(1+|\theta|)^{p+1}}
$$

and the lemma follows from (A.21) and

$$
\left\|f\left(z+\varepsilon \theta, x_{d}\right)\right\|=\left\|f\left(z, x_{d}\right)\right\| \text { in } L^{2}\left(Z ; H^{s}\right) .
$$

The next lemma gives the principal part of the symbolic calculus

Lemma A.3. For $A_{1} \in \mathcal{S}_{Z}^{t, m_{1}}, A_{2} \in \mathcal{S}_{Z}^{t, m_{2}}$, one has

$$
\left\{\begin{array}{l}
O p\left(A_{1}\right) \circ O p\left(A_{2}\right)=O p(B) \\
B=A_{1} \circ A_{2}+\varepsilon R \\
B \in \mathcal{S}_{Z}^{t, m_{1}+m_{2}}, R \in \mathcal{S}_{Z}^{t, m_{1}+m_{2}+1}
\end{array}\right.
$$

Proof. We have $O p\left(A_{1}\right) \circ O p\left(A_{2}\right)=O p(B)$ with

$$
B\left(\varepsilon, z, \zeta, x_{d}\right)=(2 \pi)^{-p} \iint e^{-i t \eta} A_{1}\left(\varepsilon, z, \zeta+\varepsilon \eta, x_{d}\right) \circ A_{2}\left(\varepsilon, z+t, \zeta, x_{d}\right) d \eta d t .
$$

Using the Taylor formula $f(\zeta+\varepsilon \eta)=f(\zeta)+\sum_{j} \varepsilon \eta_{j} \int_{0}^{1} \frac{\partial f}{\partial \zeta_{j}}(\zeta+\varepsilon s \eta) d s$ and integrating by part with respect to $t_{j}$, we get $B=A_{1} \circ A_{2}+\varepsilon R$ with

$$
R=\frac{1}{i} \sum_{j} \int_{0}^{1} d s \iint(2 \pi)^{-p} e^{-i t \eta} \frac{\partial A_{1}}{\partial \zeta_{j}}\left(\varepsilon, z, \zeta+\varepsilon s \eta, x_{d}\right) \circ \frac{\partial A_{2}}{\partial z_{j}}\left(\varepsilon, z+t, \zeta, x_{d}\right) d \eta d t .
$$

We shall verify $R \in \mathcal{S}_{Z}^{t, m_{1}+m_{2}+1}$ (the proof of $B \in \mathcal{S}_{Z}^{t, m_{1}+m_{2}}$ is similar).

If we define $B_{2}=\frac{\partial A_{2}}{\partial z_{j}} \in \mathcal{S}_{Z}^{t, m_{2}+1}$ and $B_{1} \in \mathcal{S}_{Z}^{t, m_{1}}$ by

$$
\frac{\partial A_{1}}{\partial \zeta_{j}}\left(\varepsilon, z, \zeta, x_{d}\right)=(2 \pi)^{-p} \int e^{i \theta \zeta} B_{1}\left(\varepsilon, z, \theta, x_{d}\right) d \theta
$$


we are reduce to prove

$$
\int_{0}^{1} d s \int e^{i \theta \zeta} B_{1}\left(\varepsilon, z, \theta, x_{d}\right) \circ B_{2}\left(\varepsilon, z+\varepsilon s \theta, \zeta, x_{d}\right) d \theta \in \mathcal{S}_{Z}^{t, m_{1}+m_{2}+1} .
$$

The verification of (A.25) is now easy using (A.16), the Leibniz rule for derivatives and the fact that $B_{1}$ (resp. $B_{2}$ ) is in the Schwartz space with respect to $\theta$ (resp. $\zeta$ ).

Lemma A.4. Let $\psi \in \mathcal{S}\left(\mathbb{R}^{p}\right)$ and $A \in \mathcal{S}_{Z}^{t, m}$. One has

$$
O p(A)^{*} \circ \psi\left(\frac{\varepsilon}{i} \partial z\right)=O p\left(A^{*} \psi(\zeta)\right)+\varepsilon O p(R)
$$

with $R \in \mathcal{S}_{Z}^{t, m+1}$.

Proof. We have $O p(A)^{*}=O p(B)$ with

$$
B\left(\varepsilon, z, \zeta, x_{d}\right)=(2 \pi)^{-p} \iint e^{-i t \eta} A^{*}\left(\varepsilon, z+t, \zeta+\varepsilon \eta, x_{d}\right) d \eta d t .
$$

Using the Taylor formula as before, we get the identity (A.26) with

$$
R=\frac{1}{i} \sum_{j} \int_{0}^{1} d s \int(2 \pi)^{-p} e^{-i t \eta} \frac{\partial^{2} A^{*}}{\partial z_{j} \partial \zeta_{j}}\left(\varepsilon, z+t, \zeta+\varepsilon s \eta, x_{d}\right) \psi(\zeta) d t d \eta .
$$

As in the proof of Lemma A.3, we just observe that, for $B \in \mathcal{S}_{Z}^{t, m}$,we have

$$
\int_{0}^{1} d s \int e^{i \theta \zeta} B\left(\varepsilon, z+\varepsilon s \theta, \theta, x_{d}\right) \psi(\zeta) d \theta \in \mathcal{S}_{Z}^{t, m}
$$

\section{B. Appendix}

\section{B.1. Proof of Lemmas 3.4 and 3.5}

Let $p r_{0}^{\ell}(x)$ and $p r^{\ell}\left(t, x, \tau, \xi^{\prime}\right)$ be the operators

$$
\begin{gathered}
p r_{0}^{\ell}(x)\left(\Sigma_{k} z_{k} e^{i k y}\right)=\psi\left(\frac{\left\|\ell_{x}^{\prime \prime}\right\|^{2}}{\beta^{2}}\right) z_{\ell} e^{i \ell y} \\
p r^{\ell}\left(t, x, \tau, \xi^{\prime}\right)=\psi\left(\frac{\left\|\ell_{x}^{\prime \prime}\right\|^{2}}{\beta^{2}}\right) \int_{\gamma_{\ell}(x)}\left(\lambda-\mathbb{E}\left(t, x, \tau, \xi^{\prime}\right)\right)^{-1} \frac{d \lambda}{2 i \pi}
\end{gathered}
$$

and

$$
\delta p r^{\ell}\left(t, x, \tau, \xi^{\prime}\right)=p r^{\ell}\left(t, x, \tau, \xi^{\prime}\right)-p r_{0}^{\ell}(x)
$$

Let $z=(t, x), \zeta=\left(\tau, \xi^{\prime}\right)$, and $\left(\delta p r^{\ell}\right)_{j, k}$ be the matrix of $\delta p r^{\ell}$ as in Appendix A.2. We shall prove: 
For any $\alpha, \beta, \gamma, N$, there exist $C_{\alpha, \beta, \gamma, N}$ such that

$$
\begin{aligned}
& \left\{\begin{array}{l}
(1+|\zeta|)^{\gamma}\left|\partial_{z}^{\alpha} \partial_{\zeta}^{\beta}\left(\delta p^{\ell}(z, \zeta)\right)_{j, k}\right| \leq C_{\alpha, \beta, \gamma, N} \frac{(1+|\ell|)^{\mid \alpha}}{(1+|\ell-j|+|\ell-k|)^{N}} \\
\text { for every } z, \zeta, j, k, \ell
\end{array}\right. \\
& \delta r^{\ell}(z, 0)=0 \text { for any } \ell, z .
\end{aligned}
$$

The two Lemmas 3.4 and 3.5 are consequences of the two properties (B.4, B.5), by definition of the class $\mathcal{S}^{t, m}$ (see (A.15)). In fact (B.4) implies that the series ((3.45) and (3.48)) are convergent in the class $\mathcal{S}^{t, 0}$ and $\mathcal{S}^{t,-\sigma}$.

(B.5) is obvious since $\mathbb{E}(z, 0)=\mathbb{E}_{0}(z)$ by $(3.21)$ so $\operatorname{pr}^{\ell}(z, 0)=\operatorname{pr}_{0}^{\ell}(z)$. We have also $\mathbb{E}(z, \zeta)=\mathbb{E}_{0}(z)$ for $|\zeta| \geq \alpha_{0}$ since $\mathbb{E}(z, \zeta)=\mathbb{E}_{0}(z)+\left(\begin{array}{cc}0 & 0 \\ q \theta & 0\end{array}\right)$ and $\theta(z, \zeta)=0$ for $|\zeta| \geq \alpha_{0}$ so $\delta \operatorname{pr}^{\ell}(z, \zeta)$ is compactly supported in $\zeta$ and we can forget $\gamma$ and $(1+|\zeta|)^{\gamma}$ in the proof of (B.4). We firt conjugate $\mathbb{E}\left(t, x, \tau, \xi^{\prime}\right)$ by the multiplication by $e^{i \ell y}$. We get by $(3.21,3.32,3.39)$

$$
\begin{gathered}
e^{-i \ell y} \circ \mathbb{E}(z, \zeta) \circ e^{i \ell y}=\ell_{x}^{\perp} I d+\mathbb{E}^{\ell}(z, \zeta) \\
\mathbb{E}^{\ell}(z, \zeta)=\mathbb{E}_{0}^{\ell}(z)+\left(\begin{array}{cc}
0 & 0 \\
q^{\ell} \theta & 0
\end{array}\right) \\
\mathbb{E}_{0}^{\ell}(z)\left[\Sigma w_{k} e^{i k y}\right]=\sum_{k}\left(\begin{array}{cc}
k_{x}^{\perp} & -\left\langle(k+\ell)_{x}^{\prime \prime}\right\rangle \\
\frac{\left\|(k+\ell)_{x}^{\prime \prime}\right\|^{2}}{\left\langle(k+\ell)_{x}^{\prime \prime}\right\rangle} & k_{x}^{\perp}
\end{array}\right)\left(w_{k}\right) e^{i k y} \\
\left.q^{\ell}(z, \zeta)=\left(\oplus_{k}^{\oplus\left\langle(k+\ell)_{x}^{\prime \prime}\right\rangle}\right\rangle^{-1}\right) \circ\left[a_{2}\left(x, \xi^{\prime}\right)+\sum_{j=1}^{d-1} \frac{\partial a_{2}}{\partial \xi_{j}^{\prime}}\left(x, \xi^{\prime}\right)\left(e_{j}(x) \cdot D_{y}+e_{j}(x) \cdot \ell\right)-\hat{\rho}(x, y) \tau^{2}\right] \chi .
\end{gathered}
$$

We define $\pi_{0}^{\ell}(z)=e^{-i \ell y} \circ \operatorname{pr}_{0}^{\ell}(z) \circ e^{i \ell y}$,

$$
\pi^{\ell}(z, \zeta)=e^{-i \ell y} \circ \operatorname{pr}^{\ell}(z, \zeta) \circ e^{i \ell y}, \delta \pi^{\ell}=\pi^{\ell}-\pi_{0}^{\ell} .
$$

We have

$$
\begin{gathered}
\pi_{0}^{\ell}(z)\left[\sum_{k} z_{k} e^{i k y}\right]=\psi\left(\frac{\left\|\ell_{x}^{\prime \prime}\right\|^{2}}{\beta^{2}}\right) z_{0} \\
\pi^{\ell}(z, \zeta)=\psi\left(\frac{\left\|\ell_{x}^{\prime \prime}\right\|^{2}}{\beta^{2}}\right) \int_{|\lambda|=c_{1 / 4}}\left(\lambda-\mathbb{E}^{\ell}(z, \zeta)\right)^{-1} \frac{d \lambda}{2 i \pi} .
\end{gathered}
$$


We are now reduce to prove

$$
\left\{\begin{array}{l}
\text { For any } \alpha, \beta, N, \text { there exist } C_{\alpha, \beta, N} \text { such that } \\
\left|\partial_{z}^{\alpha} \partial_{\zeta}^{\beta}\left(\delta \pi^{\ell}\right)_{j, k}(z, \zeta)\right| \leq C_{\alpha, \beta, N} \frac{(1+|\ell|)^{|\alpha|}}{(1+|j|+|k|)^{N}} \\
\text { for every } z, \zeta, j, k, \ell .
\end{array}\right.
$$

Notice that the spectrum of $\mathbb{E}_{0}^{\ell}(z)$, with $(\ell, x)$ such that $\left\|\ell_{x}^{\prime \prime}\right\| \leq 2 \beta \ll c_{1}$ can be separate in two pieces: two small eigenvalues $\pm i\left\|\ell_{x}^{\prime \prime}\right\|$ with associated eigenspace $\mathbb{C}^{2} e^{i 0 y}$, and the other part of the spectrum leaving outside the complex disk $|\lambda| \geq c_{1} / 2$. The same is true for the spectrum $\mathbb{E}^{\ell}(z, \zeta)$ (the cutt-off function $\chi(z, \zeta)$ localize $q^{\ell}$ in $\left.|\zeta| \leq 2 \alpha_{0} \ll \beta\right)$. In order to prove (B.12), we use a Grushin method.

Let $\widehat{\mathcal{A}}^{m}$ be the set of operators on $\left(\mathcal{D}^{\prime}\left(\mathbb{T}^{d}\right)\right)^{2} \oplus \mathbb{C}^{2}$ of the form

$$
L=\left(\begin{array}{ll}
A & B \\
C & D
\end{array}\right)
$$

with $A$ a $2 \times 2$ matrix with entries in $\mathcal{A}^{m}$ (see Append. A.2) B a linear map from $\mathbb{C}^{2}$ in $\left(C^{\infty}\left(\mathbb{T}^{d}\right)\right)^{2}, \mathrm{C}$ a continuous linear map from $\left(\mathcal{D}^{\prime}\left(\mathbb{T}^{d}\right)\right)^{2}$ in $\mathbb{C}^{2}$ and $D \in \mathcal{M}^{2}(\mathbb{C})$. As in Appendix A.2, we remark that $\hat{\mathcal{A}}^{0}$ is the class of bounded operators $L$ on $\mathcal{H}=\left(L^{2}\left(\mathbb{T}^{d}\right)\right)^{2} \oplus \mathbb{C}^{2}$ such that all the commutators $\left[\widetilde{\mathcal{D}}_{j_{1}}\left[\widetilde{D}_{j_{1}}, \cdots,\left[\widetilde{D}_{j, p}, L\right]\right]\right.$ are bounded on $\mathcal{H}$, with

$$
\widetilde{D}_{j}=\left(\begin{array}{cc}
\frac{1}{i} \partial_{y_{j}} & 0 \\
0 & 0
\end{array}\right) .
$$

In particular, Lemma A.1 remain valid. We denote by $\widehat{\mathcal{S}}_{V}^{m}$ the vector space of functions of $(z, \zeta) \in V, V$ open

$$
L(z, \zeta)=\left(\begin{array}{ll}
A(z, \zeta) & B(z, \zeta) \\
C(z, \zeta) & D(z, \zeta)
\end{array}\right)
$$

where $B, C, D$ are as above and depends smoothly on $(z, \zeta)$, and $A \in \mathcal{S}_{V}^{m}$. In other words, $L(z, \zeta) \in \widehat{S}^{m}$ means

$$
\forall \alpha, \beta \quad \partial_{z}^{\alpha} \partial_{\zeta}^{\beta} L \in \widehat{\mathcal{A}}^{m+|\alpha|} \text { uniformly in }(z, \zeta) \in K \Subset V .
$$

Let $j, p$ be the injection and projection

$$
\left\{\begin{array}{l}
j(w)=w e^{i 0 y} \quad: \quad \mathbb{C}^{2} \rightarrow C^{\infty}\left(\mathbb{T}^{d}\right)^{2} \\
p(f)=\oint f d y
\end{array}\right.
$$

and

$$
L^{\ell}(\lambda, z, \zeta)=\left(\begin{array}{cc}
\lambda-\mathbb{E}^{\ell}(z, \zeta) & j \\
p & 0
\end{array}\right)
$$

Then $L^{\ell}(\lambda, .,$.$) is a holomorphic family in \lambda$ with values in $\widehat{\mathcal{A}}_{V_{\ell}}^{1}$, with inverse in $\widehat{\mathcal{A}}_{V_{\ell}}^{-1}$ for $|\lambda|<\frac{c_{1}}{2}$, with $V_{\ell}=\left\{(z, \zeta) ;\left\|\ell_{x}^{\prime \prime}\right\|<2 \beta\right\}$.

Notice that in view of $(B .8, B .9), \ell_{x}^{\prime \prime}$ can be replace by a small parameter in $\mathbb{R}^{d-1}$ in both $\mathbb{E}_{0}^{\ell}, q^{\ell}$, so all the semi-norms of $\left(L^{\ell}(\lambda, z, \zeta)\right)^{-1}$ are uniform in $(\ell, x)$ such that $\left\|\ell_{x}^{\prime \prime}\right\|<2 \beta$.

Let $\mathcal{L}^{\ell}(\lambda, z, \zeta)=\left(L^{\ell}(\lambda, z, \zeta)\right)^{-1}$

$$
\mathcal{L}^{\ell}=\left(\begin{array}{ll}
A^{\ell} & B^{\ell} \\
C^{\ell} & D^{\ell}
\end{array}\right)
$$


Then $\lambda-\mathbb{E}^{\ell}(z, \zeta)$ is invertible iff $\operatorname{det}\left(D^{\ell}(\lambda, z, \zeta)\right) \neq 0$, and we have the algebraic identity

$$
\left(\lambda-\mathbb{E}^{\ell}(z, \zeta)\right)^{-1}=\left[A^{\ell}-B^{\ell}\left(D^{\ell}\right)^{-1} C^{\ell}\right](\lambda, z, \zeta) .
$$

The function $A^{\ell}$ is holomorphic in $\lambda \in\left\{|z|<\frac{c_{1}}{2}\right\}$ so we get by (B.11)

$$
\pi^{\ell}(z, \zeta)=-\psi\left(\frac{\left\|\ell_{x}^{\prime \prime}\right\|^{2}}{\beta^{2}}\right) \int_{|\lambda|=\frac{c_{1}}{4}}\left(B^{\ell}\left(D^{\ell}\right)^{-1} C^{\ell}\right)(\lambda, z, \zeta) \frac{d \lambda}{2 i \pi} .
$$

This implies that the estimate (B.12) holds true for $\pi^{\ell}$, hence for $\delta \pi^{\ell}$ ((B.12) is obvious for $\left.\pi_{0}^{\ell}\right)$.

\section{B.2. Proof of Lemma 3.6}

One has $\left[p r\left(t, x, \tau, \xi^{\prime}\right), \mathbb{E}\left(t, x, \tau, \xi^{\prime}\right)\right] \equiv 0, p r \in \mathcal{S}^{t, 0}, \mathbb{E} \in \mathcal{S}^{t, 1}$, so Lemma A.3 implies $[O p(p r), O p(\mathbb{E})] \in \varepsilon \mathcal{S}^{t, 2}$. In fact, the more precise estimate $[O p(p r), O p(\mathbb{E})] \in \varepsilon \mathcal{S}^{t, 1}$ holds true. To see this, we just observe that we have $\mathbb{E}-\mathbb{E}_{0} \in \mathcal{S}^{t, 0} ;$ from the definitions $\left((3.21)\right.$ and (3.33)) we get $\partial_{\zeta} \mathbb{E}_{0}=0, \partial_{z} \mathbb{E}_{0} \in \mathcal{S}^{t, 1}$ and the result follows from the symbolic calculus formulas (A.23) and (A.24). We then deduce from Lemma 3.1 that $F^{\varepsilon, I}=O p(I d-p r)\left(F^{\varepsilon}\right)$ satisfies the following equation

$$
\frac{\varepsilon}{i} \frac{\partial}{\partial x_{d}} F^{\varepsilon, I}+O p(\mathbb{E}) F^{\varepsilon, I}=G^{\varepsilon, I}
$$

where, $F^{\varepsilon, I}$ and $G^{\varepsilon, I}$ are such that

$$
\left\{\begin{array}{l}
\sup _{\varepsilon}\left\|F^{\varepsilon, I} ; L^{2}\left(U ; \mathcal{H}^{s_{0}}\right)\right\|<+\infty \\
\sup _{\varepsilon} \varepsilon^{-1}\left\|G^{\varepsilon, I} ; L^{2}\left(U, \mathcal{H}^{s_{0}-1}\right)\right\|<+\infty .
\end{array}\right.
$$

We shall first modified $\mathbb{E}$ in (B.21) in order to work with an elliptic equation. Let us define $\widetilde{p r}_{0}(x)$ and $\widetilde{p r}\left(t, x, \tau, \xi^{\prime}\right)$ by formulas (3.44) and (3.45) with $\psi\left(\frac{4\left\|\ell^{\prime \prime} x\right\|^{2}}{\beta^{2}}\right)$ instead of $\psi\left(\frac{\left\|\ell^{\prime \prime} x\right\|^{2}}{\beta^{2}}\right)$. One has

$$
\widetilde{p r} \circ(I d-p r)\left(t, x, \tau, \xi^{\prime}\right) \equiv 0
$$

and by the proof of Lemma 3.4 one gets

$$
\widetilde{p r}=\widetilde{p r}{ }_{0}+\delta \widetilde{p r}, \delta \widetilde{p r} \in \mathcal{M}_{2,2}\left(\mathcal{S}^{t, 0}\right),\left.\delta \widetilde{p r}\right|_{\xi^{\prime}=\tau=0}=0 .
$$

Let $\chi\left(\tau, \xi^{\prime}\right) \in C_{0}^{\infty}\left(|\tau|+\left|\xi^{\prime}\right|<2\right)$ equal to 1 near $\left(|\tau|+\left|\xi^{\prime}\right| \leq 1\right), \chi_{\alpha_{0}}\left(\tau, \xi^{\prime}\right)=\chi\left(\left(\tau, \xi^{\prime}\right) / \alpha_{0}\right)$ and let $K(x)$ be the operator on the torus

$$
K(x)\left(\Sigma_{\ell} z_{\ell} e^{i \ell y}\right)=\sum_{\ell} \psi\left(\frac{16\left\|\ell_{x}^{\prime \prime}\right\|^{2}}{\beta^{2}}\right) \frac{z_{\ell}}{\left\langle\ell_{x}^{\prime \prime}\right\rangle} e^{i \ell y}
$$

Let us define $\widetilde{\mathbb{E}}$ by the formula

$$
\left\{\begin{array}{l}
\widetilde{\mathbb{E}}=\widetilde{\mathbb{E}}_{0}+\delta \widetilde{\mathbb{E}} \\
\widetilde{\mathbb{E}}_{0}=\mathbb{E}_{0}+\left(\begin{array}{cc}
0 & 0 \\
K & 0
\end{array}\right) \\
\delta \widetilde{\mathbb{E}}=\left[\left(\begin{array}{cc}
0 & 0 \\
q \theta & 0
\end{array}\right)+\left(\begin{array}{cc}
0 & 0 \\
K & 0
\end{array}\right) \circ \delta \widetilde{p r}\right] \chi_{\alpha_{0}} .
\end{array}\right.
$$


One has $\theta \chi_{\alpha_{0}} \equiv \theta$ and $\left(\begin{array}{cc}0 & 0 \\ K & 0\end{array}\right) \circ \widetilde{p r}_{0}=\left(\begin{array}{cc}0 & 0 \\ K & 0\end{array}\right)$, which implies

$$
\widetilde{\mathbb{E}}=\mathbb{E}+\left(\begin{array}{cc}
0 & 0 \\
K & 0
\end{array}\right) \circ \widetilde{p r} \chi_{\alpha_{0}}+\left(\begin{array}{cc}
0 & 0 \\
K & 0
\end{array}\right)\left(1-\chi_{\alpha_{0}}\right) .
$$

One has $\left(1-\chi_{\alpha_{0}}\right) F^{\varepsilon, I} \in \varepsilon L^{2}\left(U ; \mathcal{H}^{s_{0}-1}\right)$ so using (B.21, B.23) and Lemma A.3, one gets

$$
\left\{\begin{array}{l}
{\left[\frac{\varepsilon}{i} \frac{\partial}{\partial x_{d}}+O p(\widetilde{\mathbb{E}})\right] F^{\varepsilon, I}=\widetilde{G}^{\varepsilon, I}} \\
\sup _{\varepsilon} \varepsilon^{-1}\left\|\widetilde{G}^{\varepsilon, I} ; L^{2}\left(U, \mathcal{H}^{s_{0}-1}\right)\right\|<+\infty
\end{array}\right.
$$

Notice that $\widetilde{\mathbb{E}}_{0}$ is a diagonal operator

$$
\left\{\begin{array}{l}
\widetilde{\mathbb{E}}_{0}\left(\Sigma z_{\ell} e^{i \ell y}\right)=\sum_{\ell} \widetilde{\mathbb{E}}_{0, \ell}\left(z_{\ell}\right) e^{i \ell y} \\
\widetilde{\mathbb{E}}_{0, \ell}=\left(\begin{array}{cc}
\ell_{x}^{\perp} & -\left\langle\ell_{x}^{\prime \prime}\right\rangle \\
\frac{\left\|\ell_{x}^{\prime \prime}\right\|^{2}+\psi\left(\frac{16\left\|\ell_{x}^{\prime \prime}\right\|^{2}}{\beta^{2}}\right)}{\left\langle\ell_{x}^{\prime \prime}\right\rangle} & \ell_{x}^{\perp}
\end{array}\right) .
\end{array}\right.
$$

The eigenvalues of $\widetilde{\mathbb{E}}_{0, \ell}$ are

$$
\widetilde{\lambda}_{\ell}^{ \pm}=\ell_{x}^{\perp} \pm i\left(\left\|\ell_{x}^{\prime \prime}\right\|^{2}+\psi\left(\frac{16\left\|\ell^{\prime \prime} x\right\|^{2}}{\beta^{2}}\right)\right)^{1 / 2}
$$

In particular, one has with $0<c_{1}<c_{2}$

$$
c_{1}\left\langle\ell_{x}^{\prime \prime}\right\rangle \leq\left|\operatorname{Im} \widetilde{\lambda}_{\ell}^{ \pm}\right| \leq c_{2}\left\langle\ell_{x}^{\prime \prime}\right\rangle .
$$

We choose the associated eigenvectors

$$
e_{\ell}^{ \pm}(x)=\left[\begin{array}{c}
\frac{-\left\langle\ell_{x}^{\prime \prime}\right\rangle}{\lambda_{\ell}^{ \pm}(x)-\ell_{x}^{\frac{1}{x}}} \\
1
\end{array}\right]
$$

The map $J_{0}(x)$ defined by

$$
J_{0}(x)\left(\sum_{\ell}\left(\begin{array}{l}
z_{\ell}^{+} \\
z_{\ell}^{-}
\end{array}\right) e^{i \ell y}\right)=\sum_{\ell}\left(z_{\ell}^{+} e_{\ell}^{+}(x)+z_{\ell}^{-} e_{\ell}^{-}(x)\right) e^{i \ell y}
$$

is then an isomorphism of $\mathcal{H}^{s}$ for any $s$.

Let $D^{ \pm}$be the operators

$$
D^{ \pm}\left(\Sigma z_{\ell} e^{i \ell y}\right)=\Sigma \widetilde{\lambda}_{\ell}^{ \pm} z_{\ell} e^{i \ell y}
$$

By construction, one has

$$
J_{0}^{-1} \widetilde{\mathbb{E}}_{0} J_{0}=\left(\begin{array}{cc}
D^{+} & 0 \\
0 & D^{-}
\end{array}\right)
$$


Lemma B.1. If $\alpha_{0}$ is small enough, there exist $\delta B, \delta C, \delta D^{+}, \delta D^{-}$in $\mathcal{S}^{t, 0}$, with support in $\left\{|\tau|+\left|\xi^{\prime}\right| \leq 2 \alpha_{0}\right\}$ vanishing on $\xi^{\prime}=0, \tau=0$ such that the following identity holds true

$$
\left(\begin{array}{cc}
I d & \delta B \\
\delta C & I d
\end{array}\right)^{-1} J_{0}^{-1} \widetilde{\mathbb{E}} J_{0}\left(\begin{array}{cc}
I d & \delta B \\
\delta C & I d
\end{array}\right)=\left(\begin{array}{cc}
D^{+}+\delta D^{+} & 0 \\
0 & D^{-}+\delta D^{-}
\end{array}\right) .
$$

Proof. By formulas (B.26, B.35), one has

$$
J_{0}^{-1} \widetilde{\mathbb{E}} J_{0}=\left(\begin{array}{cc}
D^{+} & 0 \\
0 & D^{-}
\end{array}\right)+\delta M, \delta M=\left(\begin{array}{ll}
\delta M_{1} & \delta M_{2} \\
\delta M_{3} & \delta M_{4}
\end{array}\right)
$$

where $\delta M_{j} \in \mathcal{S}^{t, 0}$, vanishes on $\xi^{\prime}=0, \tau=0$, and has support in $\left\{|\tau|+\left|\xi^{\prime}\right| \leq 2 \alpha_{0}\right\}$. Equation (B.36) is then equivalent to the following system of equations

$$
\left\{\begin{array}{l}
\delta M_{1}+\delta M_{2} \delta C=\delta D_{+} \\
\delta M_{4}+\delta M_{3} \delta B=\delta D_{-} \\
\delta M_{2}+\delta M_{1} \delta B+D^{+} \delta B=\delta B D^{-}+\delta B \delta D^{-} \\
\delta M_{3}+\delta M_{4} \delta C+D^{-} \delta C=\delta C D^{+}+\delta C \delta D^{+}
\end{array}\right.
$$

We are thus reduce to solve the equation, with unknown $\delta B \in \mathcal{S}^{t, 0}$

$$
\left\{\begin{array}{l}
D^{+} \delta B-\delta B D^{-}+\delta M_{1} \delta B+\delta M_{2}-\delta B \delta M_{4}-\delta B \delta M_{3} \delta B=\phi(\delta B, \delta M) \\
\phi(\delta B, \delta M)=0
\end{array}\right.
$$

Let $\mathcal{E}_{x}, \mathcal{E}$ be the Banach space of operators on the torus: $\left(A_{\ell, k}=\oint_{\mathbb{T}^{d}}\left(A e^{i k y}\right) e^{-i \ell y}\right)$

$$
\begin{gathered}
\mathcal{E}=\left\{A ;\|A ; \mathcal{E}\|=\sup _{\ell, k}\left|A_{\ell, k}\right|(1+|\ell-k|)^{N_{0}}<+\infty\right\} \\
\mathcal{E}_{x}=\left\{A ;\left\|A ; \mathcal{E}_{x}\right\|=\sup _{\ell, k}\left|A_{\ell, k}\right|(1+|\ell-k|)^{N_{0}}\left|\tilde{\lambda}_{\ell}^{+}(x)-\tilde{\lambda}_{k}^{-}(x)\right|<+\infty\right\}
\end{gathered}
$$

where $N_{0}$ is given, $N_{0} \geq d+1$. By (B.30, B.31), the injection $\mathcal{E}_{x} \hookrightarrow \mathcal{E}$ is continuous, and the map $\left(A_{1}, A_{2}\right)$ $\rightarrow A_{1} A_{2}$ is continuous on $\mathcal{E}$ by the choice of $N_{0}$.

We shall first verify that (B.38) has a unique small solution $\delta B \in \mathcal{E}_{x}$, for $\left(t, \tau, x, \xi^{\prime}\right)$ fixed, if $\alpha_{0}$ is small enough. By construction, one has

$$
\left(D^{+} \delta B-\delta B D^{-}\right)_{\ell, k}=\left(\widetilde{\lambda}_{\ell}^{+}-\widetilde{\lambda}_{k}^{-}\right)(\delta B)_{\ell, k}
$$

so $\delta B \mapsto D^{+} \delta B-\delta B D^{-}$is an isomorphism of $\mathcal{E}_{x}$ onto $\mathcal{E}$. The map $(\delta B, \delta M) \rightarrow \phi(\delta B, \delta M)$ is differentiable from $\mathcal{E}_{x} \times(\mathcal{E})^{4}$ to $\mathcal{E}$ and satisfies

$$
\frac{\partial}{\partial \delta B} \phi(0,0)=D^{+}(\cdot)-(\cdot) D^{-} \quad \phi(0,0)=0 .
$$

By the implicit function theorem, the equation $\phi(\delta B, \delta M)=0$ has thus a unique small solution $\delta B \in \mathcal{E}_{x}$, provide $\|\delta M ; \mathcal{E}\|$ is small. Using (B.26) ( $q$ and $\delta \widetilde{p r}$ vanish on $\left.\xi^{\prime}=\tau=0\right)$ and $\chi_{\alpha_{0}}\left(\tau, \xi^{\prime}\right)=\chi\left(\frac{\left(\tau, \xi^{\prime}\right)}{\alpha_{0}}\right)$ one gets the estimate $\left\|\delta M_{j} ; \mathcal{E}\right\| \leq C^{t e} \alpha_{0}$. This shows the existence of $\delta B$ solution of (B.38). 
It remains to prove that for any fixed $z=(t, x), \zeta=(\tau, \xi)$, we have

$$
\forall N, \exists C_{N} \quad\left|(\delta B)_{\ell, k}(z, \zeta)\right| \leq \frac{C_{N}}{(1+|\ell-k|)^{N}}
$$

and that the functions $(z, \zeta) \mapsto(\delta B)_{\ell, k}(z, \zeta)$ are smooth and satisfy

$$
\begin{aligned}
& \forall \alpha, \beta, \gamma, N, \exists C \quad \forall z, \zeta, \ell, k \\
& \left|(1+|\zeta|)^{\gamma} \partial_{z}^{\alpha} \partial_{\zeta}^{\beta}(\delta B)_{\ell, k}(z, \zeta)\right| \leq C \frac{(1+|\ell|)^{|\alpha|}}{(1+|\ell-k|)^{N}} .
\end{aligned}
$$

Let $\nabla_{i}=\frac{1}{i} \frac{\partial}{\partial y_{i}}$ a derivation on the torus. The commutator $\left[\nabla_{i}, \delta B\right]$ satisfies the linear equation

$$
\begin{cases} & \mathcal{L}\left(\left[\nabla_{i}, \delta B\right]\right) \in \mathcal{E} \\ & \mathcal{L}(u)=D^{+} u-u D^{-}+\delta M_{1} u-u \delta M_{4}-u \delta M_{3} \delta B-\delta B \delta M u\end{cases}
$$

The linear map $\mathcal{L}$ is an isomorphism of $\mathcal{E}_{x}$ onto $\mathcal{E}$ provide $\left\|\delta M_{j} ; \mathcal{E}\right\|$ (hence $\|\delta B ; \mathcal{E}\|$ ) is small enough; decreasing $\alpha_{0}$ if necessary, we find that (B.45) admits a unique solution $\left[\nabla_{i}, \delta B\right] \in \mathcal{E}_{x} \hookrightarrow \mathcal{E}$. By iteration, all the commutators $\left[\nabla_{i 1},\left[\nabla_{i 2}, \cdots\left[\nabla_{1 k}, \delta B\right] \cdots\right]\right.$ belongs to $\mathcal{E}_{x}$ so (B.43) holds true. By construction, the functions $(\delta B)_{\ell, k}(z, \zeta)$ are smooth and compactly supported in $\left\{|\zeta| \leq 2 \alpha_{0}\right\}$. For any $m \geq 0$, let $\mathcal{A}^{m}, \mathcal{A}_{x}^{m}$ be the vector spaces

$$
\begin{gathered}
\mathcal{A}^{m}=\left\{A ; \forall N, \exists C_{N}\left|A_{\ell, k}\right| \leq C_{N} \frac{(1+|\ell|)^{m}}{(1+|\ell-k|)^{N}}\right\} \\
\mathcal{A}_{x}^{m}=\left\{A ; \forall N, \exists C_{N}\left|A_{\ell, k}\right| \leq \frac{C_{N}}{\left|\widetilde{\lambda}_{\ell}^{+}(x)-\widetilde{\lambda}_{k}^{-}(x)\right|} \frac{(1+|\ell|)^{m}}{(1+|\ell-k|)^{N}}\right\} .
\end{gathered}
$$

In order to prove (B.44), we differentiate (B.38) with respect to $(z, \zeta)$ and we are reduce to verify that the following assertion holds true

$$
\left\{\begin{array}{l}
\text { There exist } \beta>0 \text { such that for } \delta M_{j}, \delta B \in \mathcal{A}^{0}, \\
\text { with } \sum_{j}\left\|\delta M_{j} ; \mathcal{E}\right\|+\|\delta B ; \mathcal{E}\| \leq \beta, \text { the map } u \mapsto \mathcal{L}(u) \\
\text { is an isomorphism of } \mathcal{A}_{x}^{m} \text { onto } \mathcal{A}^{m} \text { for any } m \geq-1 .
\end{array}\right.
$$

(Here we use the fact that $A \mapsto\left(\partial_{x}^{\alpha} D^{+}\right) A-A\left(\partial_{x}^{\alpha} D^{-}\right)$maps $A_{x}^{m}$ into $\mathcal{A}^{m+|\alpha|}$ for any $\alpha$ : it is a consequence of the estimates $\left|\widetilde{\lambda}_{\ell}^{+}(x)-\widetilde{\lambda}_{k}^{-}(x)\right| \geq C^{t e}\left(\left\langle\ell_{x}^{\prime \prime}\right\rangle+\left\langle k_{x}^{\prime \prime}\right\rangle\right)$ and for $\left.|\alpha| \geq 1 \quad\left|\partial_{x}^{\alpha} \widetilde{\lambda}_{\ell}^{ \pm}(x)\right| \leq C_{\alpha}(1+|\ell|)^{|\alpha|}\left\langle\ell_{x}^{\prime \prime}\right\rangle.\right)$

Let us first verify that (B.48) holds true for $m=0$. We remark that $\mathcal{A}^{0}$ (resp. $\mathcal{A}_{x}^{0}$ ) is the set of operators $A \in \mathcal{E}\left(\operatorname{resp} . \mathcal{E}_{x}\right)$ such that all the commutators $\left[\nabla_{i_{1}}\left[\nabla_{i_{1}}\left[\nabla_{i_{2}}, \cdots,\left[\nabla_{i_{p}}, A\right]\right]\right.\right.$ belongs to $\mathcal{E}$ (resp. $\mathcal{E}_{x}$ ). For $\beta$ small $\mathcal{L}$ is an isomorphism between $\mathcal{E}_{x}$ and $\mathcal{E}$ and for $u \in \mathcal{E}_{x}, v \in \mathcal{E}$ such that $\mathcal{L}(u)-v=0$, one has $\mathcal{L}\left(\left[\nabla_{i}, u\right]\right)-\left[\nabla_{i}, v\right] \in \mathcal{E}$. Therefore (B.48) holds true for $m=0$, and by the same argument for $m=-1$. We now fixe $\beta$ and we proceed by induction on $m \geq 1$; let us assume that (B.48) holds true for $-1 \leq m^{\prime} \leq m-1$. Let $\Lambda$ be the operator on the torus $\Lambda\left(\Sigma z_{\ell} e^{i \ell y}\right)=\Sigma(1+|\ell|) z_{\ell} e^{i \ell y}$. We have $\mathcal{L}(u)=D^{+} u-u D^{-}+p u+u q$ with $p, q \in \mathcal{A}^{0}$, so $[\Lambda, p] \in \mathcal{A}^{0}$; from $\left[\Lambda, D^{ \pm}\right]=0$, we get $\mathcal{L}(\Lambda w)-\Lambda \mathcal{L}(w)=[p, \Lambda] w$. Let $J: \mathcal{A}^{m} \rightarrow \mathcal{A}_{x}^{m}$ the map

$$
J(v)=\Lambda \mathcal{L}^{-1}\left(\Lambda^{-1} v\right)+\mathcal{L}^{-1}\left([\Lambda, p] \mathcal{L}^{-1}\left(\Lambda^{-1} v\right)\right)
$$

where $\mathcal{L}^{-1}: \mathcal{A}^{m-1} \rightarrow \mathcal{A}_{x}^{m-1}$ is the inverse map of $\mathcal{L}$. We have $\mathcal{L} \circ J(v) \equiv v$, and it remains to show that $u \in \mathcal{A}_{x}^{m}$, and $\mathcal{L}(u)=0$ imply $u=0$ : we have $\left[\Lambda^{-1}, p\right] \in \mathcal{A}^{-2}$ so $\mathcal{L}(u)=0 \Rightarrow \mathcal{L}\left(\Lambda^{-1} u\right)=\left[p, \Lambda^{-1}\right] u \in \mathcal{A}^{m-2}$ $\Rightarrow \Lambda^{-1} u \in \mathcal{A}_{x}^{m-2} \Rightarrow u \in \mathcal{A}_{x}^{m-1}$ and we get $u=0$. 
Lemma B.2. Let $U_{0}=\left\{z \in \mathbb{R}^{p},|z| \leq r_{0}\right\}$, and $U=U_{0} \times\left[0, r_{1}\right]$ with $r_{0}, r_{1}>0$. For any $\ell \in \mathbb{Z}^{d}$, let $\lambda_{\ell}\left(z, x_{d}\right) \in C^{0}(U ; \mathbb{C})$ be given continuous functions such that

$$
\begin{aligned}
& \exists c_{0}>0, \exists c_{1}>1, \forall \ell, \forall z, x_{d} \\
& \operatorname{Im} \lambda_{\ell}\left(z, x_{d}\right) \geq c_{0} \text { and } \frac{\left|\lambda_{\ell}\left(z, x_{d}\right)\right|}{1+|\ell|} \in\left[\frac{1}{c_{1}}, c_{1}\right] .
\end{aligned}
$$

Let $D\left(z, x_{d}\right)$ be the operator on the torus

$$
D\left(z, x_{d}\right)\left[\Sigma u_{\ell} e^{i \ell y}\right]=\Sigma \lambda_{\ell}\left(z, x_{d}\right) u_{\ell} e^{i \ell y} .
$$

Let $\sigma \in \mathbb{R}$ be given, and for $\varepsilon \in] 0,1], B_{\varepsilon}\left(x_{d}\right)$ a family of bounded operator on $E^{\sigma}=L^{2}\left(U_{0}, H^{\sigma}\left(\mathbb{T}^{d}\right)\right)$ such that

$$
\left\{\begin{array}{l}
\text { i) } \quad \forall f \in E^{\sigma}, \forall \varepsilon \quad x_{d} \mapsto B_{\varepsilon}\left(x_{d}\right)[f] \text { is a continuous function } \\
\text { of } x_{d} \in\left[0, r_{1}\right] \text { with values in } E^{\sigma} \\
\text { ii) } \exists \delta, \forall \varepsilon, \forall x_{d} \quad\left\|B_{\varepsilon}\left(x_{d}\right) ; E^{\sigma} \rightarrow E^{\sigma}\right\| \leq \delta
\end{array}\right.
$$

Then, for $\delta<c_{0}$ the Cauchy problem

$$
\left\{\begin{array}{l}
\left.\left[\frac{\varepsilon}{i} \frac{d}{d x_{d}}-\left(D+B_{\varepsilon}\right)\right]\left(u^{\varepsilon}\left(x_{d}\right)\right)=0 x_{d} \in\right] 0, r_{1}[ \\
u^{\varepsilon}(0)=u_{0} \in E^{\sigma}
\end{array}\right.
$$

admits a solution $u^{\varepsilon} \in C^{0}\left(\left[0, r_{1}\right], E^{\sigma}\right) \cap C^{1}\left(\left[0, r_{1}\right], E^{\sigma-1}\right)$ such that

$$
\left\|u^{\varepsilon}\left(x^{d}\right), E^{\sigma}\right\| \leq\left\|u_{0}, E^{\sigma}\right\| e^{-\left(c_{0}-\delta\right) x_{d} / \varepsilon} .
$$

Proof. We first observe that the assumption (B.50) implies that $D$ maps $C^{0}\left(\left[0, r_{1}\right], E^{\sigma}\right)$ onto $\left.C^{0}\left[0, r_{1}\right], E^{\sigma-1}\right)$ for any $\sigma$. We have $\left\|v ; E^{\sigma}\right\|=\left\|\left(1+\left|D_{y}\right|^{2}\right)^{\sigma / 2} v ; E^{0}\right\|$ and $\left[D,\left(1+\left|D_{y}\right|^{2}\right)^{\sigma / 2}\right]=0$, so if one replace $B_{\varepsilon}$ by $\left(1+\left|D_{y}\right|^{2}\right)^{\sigma / 2} B_{\varepsilon}\left(1+\left|D_{y}\right|^{2}\right)^{-\sigma / 2}$, we are reduce to the case $\sigma=0$. For any $L$, let $\pi_{L}$ be the orthogonal projector $\pi_{L}\left(\Sigma u_{\ell} e^{i \ell y}\right)=\sum_{|\ell| \leq L} u_{\ell} e^{i \ell y}$. The equation

$$
\left\{\begin{array}{l}
\left(\frac{\varepsilon}{i} \frac{d}{d x_{d}}-\pi_{L}\left(D+B_{\varepsilon}\right) \pi_{L}\right) u_{L}^{\varepsilon}\left(x_{d}\right)=0 \\
u_{L}^{\varepsilon}(0)=\pi_{L}\left(u_{0}\right)
\end{array}\right.
$$

is an ordinary differential equation in the Hilbert space $=L^{2}\left(U_{0}, \underset{|\ell| \leq L}{\oplus} \mathbb{C} e^{i \ell y}\right)=E_{L} \hookrightarrow E^{s}$, so admit a unique solution $u_{L}^{\varepsilon} \in C^{1}\left(\left[0, r_{1}\right], E_{L}\right)$. It satisfies the identity,

$$
\frac{d}{d x_{d}}\left\|u_{L}^{\varepsilon}\right\|^{2}=2 \operatorname{Re}\left(\frac{i}{\varepsilon} D u_{L}^{\varepsilon} \mid u_{L}^{\varepsilon}\right)+2 \operatorname{Re}\left(i / \varepsilon \pi_{L} B_{\varepsilon} \pi_{L} u_{L}^{\varepsilon} \mid u_{L}^{\varepsilon}\right)
$$

so we get using (B.50) and (B.52) $\frac{d}{d x_{d}}\left\|u_{L}^{\varepsilon}\right\|^{2} \leq \frac{-2}{\varepsilon}\left(c_{0}-\delta\right)\left\|u_{L}^{\varepsilon}\right\|^{2}$, which implies

$$
\left\|u_{L}^{\varepsilon}\left(x_{d}\right), E^{0}\right\| \leq\left\|u_{0}, E^{0}\right\| e^{-\left(c_{0}-\delta\right) \frac{x_{d}}{\varepsilon}} .
$$

Therefore $u_{L}^{\varepsilon}$ is bounded in $L^{2}\left(\left[0, r_{1}\right], E^{\sigma}\right) \cap H^{1}\left(\left[0, r_{1}\right], E^{\sigma-1}\right)=F$ for fixed $\varepsilon$ so we can extract a subsequence $u_{L_{k}}^{\varepsilon}$ so that $u_{L_{k}}^{\varepsilon} \stackrel{\text { weak }}{\rightarrow} u^{\varepsilon}$ in $F$ and $u^{\varepsilon}$ satisfies (B.53). In particular we have $\frac{\varepsilon}{i} \frac{d}{d x_{d}} u_{\varepsilon}-D u^{\varepsilon}=B_{\varepsilon} u^{\varepsilon} \in L^{2}\left(\left[0, r_{1}\right], E^{\sigma}\right)$ so $u^{\varepsilon} \in C^{0}\left(\left[0, r_{1}\right], E^{\sigma}\right) \cap C^{1}\left(\left[0, r_{1}\right], E^{\sigma-1}\right)$. The estimate (B.54) is then a consequence of (B.57). 
We can now achieve the verification of Lemma 3.6. We choose a tangential scalar o.p.d. $Q_{2}$ equal to $I d$ near the support of $Q_{1}$ and with essential support closed to $\rho_{0}$, and we define $T=Q_{2} \delta \widetilde{\mathbb{E}} Q_{2}$. Then $O p(T)\left(x_{d}\right)$ acts on $L^{2}\left(U_{0}, H^{\sigma}\right), \forall \sigma$. Using (B.28), we still have

$$
\left[\frac{\varepsilon}{i} \frac{\partial}{\partial x_{d}}+O p\left(\widetilde{\mathbb{E}}_{0}+T\right)\right] F^{\varepsilon, I} \in \varepsilon L^{2}\left(U, \mathcal{H}^{s_{0}-1}\right) .
$$

We then apply Lemma B.1 to $\widetilde{\mathbb{E}}_{0}^{*}+T^{*}$ instead of $\widetilde{\mathbb{E}}$; let $I_{0}(x)$ be the map

$$
I_{0}(x)\left(\sum_{\ell}\left(\begin{array}{c}
z_{\ell}^{+} \\
z_{\ell}^{-}
\end{array}\right) e^{i \ell y}\right)=\sum_{\ell}\left[z_{\ell}^{+}\left(\begin{array}{c}
\frac{-\tilde{\lambda}_{\ell}^{+}(x)+\ell_{x}^{\perp}}{\left\langle\ell_{x}^{\prime \prime}\right\rangle} \\
1
\end{array}\right)+z_{\ell}^{-}\left(\begin{array}{c}
\frac{-\tilde{\lambda}_{\ell}^{-}(x)+\ell_{x}^{\perp}}{\left\langle\ell_{x}^{\prime \prime}\right\rangle} \\
1
\end{array}\right)\right] e^{i \ell y} .
$$

We get the existence of $\delta B, \delta C, \delta D^{+}, \delta D^{-}$in $\mathcal{S}^{t, 0}$ such that, with

$$
I=I_{0}\left(\begin{array}{cc}
I d & \delta B \\
\delta C & I d
\end{array}\right)
$$

One has

$$
I^{-1}\left(\widetilde{\mathbb{E}}_{0}^{*}+T^{*}\right) I=\left(\begin{array}{cc}
D^{+}+\delta D^{+} & 0 \\
0 & \left(D^{-}+\delta D^{-}\right)
\end{array}\right)
$$

Moreover, by the proof of Lemma B.1, and the fact that $\lim _{\alpha_{0} \rightarrow 0}\left\|\left(\left|\xi^{\prime}\right|+|\tau|\right) \chi_{\alpha_{0}}\left(\xi^{\prime}, \tau\right)\right\|_{L^{\infty}}=0$, we may suppose that the norm of the tangential operators $\delta D^{ \pm}\left(x_{d}\right)$ acting on $L^{2}\left(U_{0}, H^{\left|s_{0}\right|+1}\right)$ is as small as we want. Taking in account the lower bound (B.31) $-\operatorname{Im} \lambda_{\ell}^{-}(x) \geq c_{1}\left\langle\ell_{x}^{\prime \prime}\right\rangle \geq c_{1}$, we can apply Lemma B.2. For every $h \in L^{2}\left(U_{0}, H^{\left|s_{0}\right|+1}\right)$ we get $v^{\varepsilon} \in L^{2}\left(U_{0} \times\left[0, r_{1}\right], H^{\left|s_{0}\right|+1}\right)$ such that

$$
\left\{\begin{array}{l}
{\left[\frac{\varepsilon}{i} \frac{\partial}{\partial x_{d}}+O p\left(D^{-}+\delta D^{-}\right)\right] v^{\varepsilon}=0} \\
v^{\varepsilon} \mid x_{d}=0=\varepsilon^{-1 / 2} h \\
\sup _{\varepsilon}\left\|v^{\varepsilon}, L^{2}\left(U_{0} \times\left[0, r_{1}\right] ; H^{\left|s_{0}\right|+1}\right)\right\| \leq C^{t e}\left\|h ; L^{2}\left(U_{0} ; H^{\left|s_{0}\right|+1}\right)\right\| .
\end{array}\right.
$$

We put $\underline{v}^{\varepsilon}=\left[\begin{array}{c}0 \\ v^{\varepsilon}\end{array}\right]$.

We choose $\theta\left(x_{d}\right) \in C_{0}^{\infty}(]-r_{1}, r_{1}[)$ equal to 1 near zero. We denote by $\langle\mid\rangle$ the duality between $L^{2}\left(V_{0}, H^{\sigma}\right)$ and $L^{2}\left(V_{0}, H^{-\sigma}\right)$. We have by (B.58)

$$
\int_{0}^{\infty}\left\langle\left(\frac{\varepsilon}{i} \partial_{x_{d}}+O p\left(\widetilde{\mathbb{E}}_{0}+T\right)\right) F^{\varepsilon, I} \mid \theta\left(x_{d}\right) O p(I) \underline{v}^{\varepsilon}\right\rangle \in 0(\varepsilon)\|h\|
$$

We integrate by part, taking into account Lemma A.4 and $\left\|\theta^{\prime}\left(x_{d}\right) O p(I) \underline{v}^{\varepsilon} ; L^{2}\left(U, \mathcal{H}^{\left|s_{0}\right|+1}\right)\right\| \leq C^{t e}\|h\|$, we get

$$
\frac{\varepsilon}{i}\left\langle\left. F^{\varepsilon, I}\right|_{x_{d}=0}\left|O p(I) \underline{v}_{\varepsilon}\right|_{x_{d=0}}\right\rangle=\int_{0}^{\infty}\left\langle\theta\left(x_{d}\right) F^{\varepsilon, I} \mid\left(\frac{\varepsilon}{i} \partial_{x_{d}}+O p\left(\widetilde{\mathbb{E}}_{0}^{*}+T^{*}\right)\right) O p(I) \underline{v}^{\varepsilon}\right\rangle d x_{d}+0(\varepsilon\|h\|) .
$$

We have $\left\|\varepsilon\left[\frac{\partial}{\partial x_{d}} O p(I)\right] \underline{v}^{\varepsilon} ; L^{2}\left(U, \mathcal{H}^{\left|s_{0}\right|}\right)\right\| \leq C^{t e} \varepsilon\|h\|$, and the estimates

$$
\left\{\begin{array}{l}
\left|\partial_{x}^{\alpha}\left[\frac{\tilde{\lambda}_{\ell}^{ \pm}(x)-\ell_{x}^{\perp}}{\left\langle\ell_{x}^{\prime \prime}\right\rangle}\right]\right| \leq C_{\alpha}(1+|\ell|)^{|\alpha|} \quad \forall \alpha \\
\left|\partial_{x}^{\alpha} \widetilde{\mathbb{E}_{0, \ell} \mid} \leq C_{\alpha}(1+|\ell|)^{|\alpha|} \quad \forall \alpha,\right| \alpha \mid \geq 1
\end{array}\right.
$$


implies

$$
\left\{\begin{array}{l}
\left\|\left[O p\left(\widetilde{\mathbb{E}}_{0}^{*}+T^{*}\right) \circ O p(I)-O p\left(\left(\widetilde{\mathbb{E}}_{0}^{*}+T^{*}\right) I\right)\right] \underline{v}^{\varepsilon} ; L^{2}\left(U, \mathcal{H}^{\left|s_{0}\right|}\right)\right\| \leq C^{t e} \varepsilon\|h\| \\
\|\left[O p\left(\left(I\left(\begin{array}{cc}
D^{+}+\delta D^{+} & 0 \\
0 & D^{-}+\delta D^{-}
\end{array}\right)\right)-O p(I) \circ O p\left(\begin{array}{cc}
D^{+}+\delta D^{+} & 0 \\
0 & D^{-}+\delta D^{-}
\end{array}\right)\right] \underline{v^{\varepsilon}} ; L^{2}\left(U, \mathcal{H}^{\left|s_{0}\right|}\right)\left\|\leq C^{t e} \varepsilon\right\| h \| .\right.
\end{array}\right.
$$

From (B.61, B.63, B.65), we get

$$
\left|\left\langle\left. F^{\varepsilon, I}\right|_{x_{d}=0} \mid O p(I)\left[\begin{array}{l}
0 \\
h
\end{array}\right]\right\rangle\right| \leq C^{t e} \varepsilon^{1 / 2}\|h\| .
$$

If one use the definition of $I$, the fact that $h$ is arbitrary in $L^{2}\left(U_{0}, H^{\left|s_{0}\right|+1}\right)$, one get for some $D \in \mathcal{S}^{0}$

$$
\left\|O p(I d+\delta B)^{*} \operatorname{Tr}_{1}\left(F^{\varepsilon, I}\right)-O p(D) T r_{0}\left(F^{\varepsilon, I}\right) ; L^{2}\left(U_{0}, H^{s_{0}-1}\right)\right\| \leq C \varepsilon^{1 / 2} .
$$

Lemma 3.6 is then a consequence of the estimates (3.52), Lemmas A.2-A.4, and the fact for $\alpha_{0}$ small, $I d+(\delta B)^{*}$ is invertible in $\mathcal{S}^{0}$.

\section{REFERENCES}

[1] M. Avellaneda, C. Bardos and J. Rauch, Contrôlabilité exacte, homogénéisation et localisation d'ondes dans un milieu non-homogène. Asymptot. Anal. 5 (1992) 481-484.

[2] G. Allaire and C. Conca, Bloch wave homogenization and spectral asymptotic analysis. J. Math. Pures Appl. 77 (1998) 153-208.

[3] N. Burq and G. Lebeau, Mesures de défaut de compacité; applications au système de Lamé, preprint.

[4] C. Bardos, G. Lebeau and J. Rauch, Sharp sufficient conditions for the observation, control and stabilization of waves from the boundary. SIAM J. Control Optim. 30 (1992) 1024-1075.

[5] C. Castro, Boundary controllability of the one dimensional wave equation with rapidly oscillating density, preprint.

[6] C. Castro and E. Zuazua, Contrôle de l'équation des ondes à densité rapidement oscillante à une dimension d'espace. $C$. $R$. Acad. Sci. Paris 324 (1997) 1237-1242.

[7] P. Gérard, Mesures semi-classiques et ondes de Bloch, Séminaire X EDP, exposé 16 (1991).

[8] P. Gérard and E. Leichtnam, Ergodic properties of eigenfunctions for the Dirichlet problem. Duke Math. J. 71 (1993) 559-607.

[9] G. Lebeau, Contrôle de l'équation de Schrödinger. J. Math. Pures Appl. 71 (1993) 267-291.

[10] G. Lebeau, Équation des ondes amorties, Algebraic and Geometric Methods in Mathematical Physics, A. Boutet de Monvel and V. Marchenko, Eds. Kluwer Academic Publishers (1996) 73-109.

[11] R. Melrose and J. Sjöstrand, Singularities of boundary value problems I, II. Comm. Pure Appl. Math. 31 (1978) 593-617; 35 (1982) 129-168. 